J. Inst. Math. Jussieu (2021), 1-51

doi:10.1017/S1474748021000542 ～(C) The Author(s), 2021. Published by Cambridge University Press. 1 This is an Open Access article, distributed under the terms of the Creative Commons Attribution licence (http://creativecommons.org/licenses/by/4.0), which permits unrestricted re-use, distribution and reproduction, provided the original article is properly cited.

\title{
ON FUNDAMENTAL FOURIER COEFFICIENTS OF SIEGEL CUSP FORMS OF DEGREE 2
}

\author{
JESSE JÄÄSAARI ${ }^{1}$, STEPHEN LESTER@ ${ }^{1,2}$ AND ABHISHEK SAHA ${ }^{3}$ \\ ${ }^{1}$ School of Mathematical Sciences, Queen Mary University of London, \\ London E1 $4 N S, U K$ \\ (j.jaasaari@qmul.ac.uk) \\ ${ }^{2}$ Department of Mathematics, King's College London, London W2CR 2LS, UK \\ (steve.lester@kcl.ac.uk) \\ ${ }^{3}$ School of Mathematical Sciences, Queen Mary University of London, \\ London E1 $4 N S$, UK \\ (abhishek.saha@qmul.ac.uk)
}

(Received 8 January 2021; revised 18 September 2021; accepted 22 September 2021)

\begin{abstract}
Let $F$ be a Siegel cusp form of degree 2, even weight $k \geq 2$, and odd square-free level $N$. We undertake a detailed study of the analytic properties of Fourier coefficients $a(F, S)$ of $F$ at fundamental matrices $S$ (i.e., with $-4 \operatorname{det}(S)$ equal to a fundamental discriminant). We prove that as $S$ varies along the equivalence classes of fundamental matrices with $\operatorname{det}(S) \asymp X$, the sequence $a(F, S)$ has at least $X^{1-\varepsilon}$ sign changes and takes at least $X^{1-\varepsilon}$ 'large values'. Furthermore, assuming the generalized Riemann hypothesis as well as the refined Gan-Gross-Prasad conjecture, we prove the bound $|a(F, S)| \ll_{F, \varepsilon}$ $\frac{\operatorname{det}(S)^{\frac{k}{2}}-\frac{1}{2}}{(\log |\operatorname{det}(S)|)^{\frac{1}{8}-\varepsilon}}$ for fundamental matrices $S$.
\end{abstract}

Key words and phrases: Siegel modular form, Fourier coefficients, L-function, sign changes

2020 Mathematics Subject Classification: Primary 11F30

Secondary 11F $37 ; 11 \mathrm{~F} 46 ; 11 \mathrm{~F} 67$

\section{Introduction}

Let $S_{k}\left(\Gamma_{0}^{(2)}(N)\right)$ denote the space of Siegel cusp forms of degree 2 and weight $k$ with respect to the congruence subgroup $\Gamma_{0}^{(2)}(N) \subseteq \operatorname{Sp}_{4}(\mathbb{Z})$ of level $N$. Any $F \in S_{k}\left(\Gamma_{0}^{(2)}(N)\right)$ has a Fourier expansion of the form

$$
F(Z)=\sum_{S \in \Lambda_{2}} a(F, S) e^{2 \pi i \operatorname{Tr}(S Z)}, \quad Z \text { in the Siegel upper half-space, }
$$

where the set $\Lambda_{2}$ consists of symmetric, semi-integral, positive-definite $2 \times 2$ matrices $S$ - that is,

$$
\Lambda_{2}=\left\{\left(\begin{array}{cc}
a & b / 2 \\
b / 2 & c
\end{array}\right), \quad a, b, c \in \mathbb{Z}, \quad a>0, \quad d:=b^{2}-4 a c<0\right\} .
$$


For $S=\left(\begin{array}{cc}a & b / 2 \\ b / 2 & c\end{array}\right) \in \Lambda_{2}$, we $\operatorname{define} \operatorname{disc}(S)=-4 \operatorname{det}(S)=b^{2}-4 a c$. If $d=\operatorname{disc}(S)$ is a fundamental discriminant, ${ }^{1}$ then $S$ is called fundamental. A fundamental $S$ is automatically primitive (i.e., $\operatorname{gcd}(a, b, c)=1$ ). Observe that if $d$ is odd, then $S$ is fundamental if and only if $d$ is square-free. The fundamental Fourier coefficients of Siegel cusp forms are deep and highly interesting objects. These are the basic building blocks, in the sense that one cannot use the theory of Hecke operators to relate the Fourier coefficients $a(F, S)$ at these matrices to those at simpler matrices. Furthermore, fundamental Fourier coefficients are closely related to central $L$-values.

In $[56,58]$ it was proved that if $k>2$ is even and $N$ is square-free, then elements of $S_{k}\left(\Gamma_{0}^{(2)}(N)\right.$ ) (under some mild assumptions) are uniquely determined by their fundamental Fourier coefficients. More precisely, it was proved there that for $k, N$ as described, if $F \in S_{k}\left(\Gamma_{0}^{(2)}(N)\right)$ is nonzero and an eigenfunction of the $U(p)$ operators for $p \mid N$, then $a(F, S) \neq 0$ for infinitely many matrices $S$ such that $\operatorname{disc}(S)$ is odd and square-free. This nonvanishing result is crucial for the existence of good Bessel models [44, Lemma 5.1.1], and consequently was needed for removing a key assumption from theorems due to Furusawa [17], Pitale and Schmidt [46], and the third named author of the present paper $[44,54,55]$ on the degree $8 L$-function on $\mathrm{GSp}_{4} \times \mathrm{GL}_{2}$. Furthermore, there is a remarkable identity, explained in more detail in $\S 1.3$, relating squares of (weighted averages of) fundamental Fourier coefficients and central values of dihedral twists of $\mathrm{GSp}_{4}$ and $\mathrm{GL}_{2} L$-functions. Indeed, the fundamental Fourier coefficients are unipotent periods whose weighted averages are Bessel periods whose absolute squares are essentially central $L$-values of degree $8 \mathrm{~L}$-functions, via the refined Gan-Gross-Prasad conjectures [40].

Motivated by these connections, the objective of this paper is to better understand the nature of the fundamental Fourier coefficients. In particular, we investigate the following questions:

- Are there many sign changes among the fundamental Fourier coefficients?

- How large (in the sense of both lower and upper bounds) are the fundamental Fourier coefficients?

We emphasize that while these kinds of questions have been previously studied for the full sequence $a(F, S)\left(S \in \Lambda_{2}\right)$ of Fourier coefficients attached to $F$ (see [12, 22, 24] for results on sign changes and [13] for an $\Omega$-result), there appears to be virtually no previous work in the more subtle setting where one restricts to fundamental Fourier coefficients. There has also been a fair bit of work on sign changes of Hecke eigenvalues of Siegel cusp forms [14, 36, 45, 53], which can be combined with the Hecke relations [1] to deduce sign changes among the $a(F, S)$ with $\operatorname{disc}(S)=d m^{2}$, where $d$ is a fixed fundamental discriminant and $m$ varies. This should make it clear that the problem of obtaining sign changes or growth asymptotics for Fourier coefficients not associated to fundamental discriminants is of a different flavor (and relatively easier). Our focus in this

\footnotetext{
${ }^{1}$ Recall that an integer $n$ is a fundamental discriminant if either $n$ is a square-free integer congruent to 1 modulo 4 or $n=4 m$, where $m$ is a square-free integer congruent to 2 or 3 modulo 4 .
} 
paper is on the subsequence of Fourier coefficients $a(F, S)$ with $S$ restricted to matrices of fundamental discriminant, where these questions are more difficult.

\subsection{Main results}

Let $k>2$ be an even integer and $N$ be an odd square-free integer. Fix $F \in S_{k}\left(\Gamma_{0}^{(2)}(N)\right)$. If $N>1$, assume that $F$ is an eigenform for the $U(p)$ Hecke operator (see equation (50)) for the finitely many primes $p \mid N$; we make no assumptions concerning whether $F$ is a Hecke eigenform at primes not dividing the level $N$. Our main result on sign changes is as follows:

Theorem A (see Theorem 5.2). For F as before with real Fourier coefficients, one can fix $M$ such that given $\varepsilon>0$ and sufficiently large $X$, there are $\geq X^{1-\varepsilon}$ distinct odd square-free integers $n_{i} \in[X, M X]$ and associated fundamental matrices $S_{i} \in \Lambda_{2}$ with $\left|\operatorname{disc}\left(S_{i}\right)\right|=n_{i}$, such that with the $n_{i}$ ordered in increasing manner, we have $a\left(F, S_{i}\right) a\left(F, S_{i+1}\right)<0$.

Thus, Theorem A asserts that there are at least $X^{1-\varepsilon}$ (strict) sign changes among the fundamental Fourier coefficients of discriminant $\asymp X$. Interestingly, this also improves the exponent of the nonvanishing results of $[56,58]$ mentioned earlier, where it was proved that there are $\gg_{\varepsilon} X^{5 / 8-\varepsilon}$ nonvanishing fundamental Fourier coefficients of discriminant up to $X$.

Another question left unanswered in all previous works is that of lower bounds for $|a(F, S)|$ with $S$ fundamental. Let $F$ be as before and fixed. A famous (and very deep) conjecture of Resnikoff and Saldana [51] predicts that for $S$ a fundamental matrix ${ }^{2}$ in $\Lambda_{2}$,

$$
|a(F, S)| \ll_{F, \varepsilon}|\operatorname{disc}(S)|^{\frac{k}{2}-\frac{3}{4}+\varepsilon} .
$$

We prove a lower bound for many fundamental Fourier coefficients with an exponent of the same strength.

Theorem B (see Theorem 5.3). For $F$ as before, $\varepsilon>0$ fixed, and all sufficiently large $X$, there are $\geq X^{1-\varepsilon}$ distinct odd square-free integers $n \in[X, 2 X]$, with associated fundamental matrices $S_{n}$ such that $\left|\operatorname{disc}\left(S_{n}\right)\right|=n$ and

$$
\left|a\left(F, S_{n}\right)\right| \geq n^{\frac{k}{2}-\frac{3}{4}} \exp \left(\frac{1}{82} \sqrt{\frac{\log n}{\log \log n}}\right) .
$$

Theorem B tells us that there are at least $X^{1-\varepsilon}$ fundamental Fourier coefficients of discriminant $\asymp X$ whose sizes are 'large'. Incidentally, just like Theorem A, Theorem $\mathrm{B}$ also improves upon the exponent of the set of nonvanishing fundamental coefficients obtained in [58] from $5 / 8$ to 1 .

Next, we investigate upper bounds for the Fourier coefficients $|a(F, S)|$ for fundamental $S$. The best currently known bound is due to Kohnen [35], who proved that $|a(F, S)| \ll_{F, \varepsilon}$ $|\operatorname{disc}(S)|^{\frac{k}{2}-\frac{13}{36}+\varepsilon}$. This bound is quite far from the conjectured true bound (3). In fact,

${ }^{2}$ The conjecture extends to nonfundamental matrices, but then it needs to be modified slightly by excluding the Saito-Kurokawa lifts. 
even if one were to assume the generalized Lindelöf hypothesis, one only obtains the upper bound $\ll_{F, \varepsilon}|\operatorname{disc}(S)|^{\frac{k}{2}-\frac{1}{2}+\varepsilon}$ (as explained later). Thus, the exponent $\frac{k}{2}-\frac{1}{2}$ appears to be a natural barrier. By using probabilistic methods and assuming the generalized Riemann hypothesis $(\mathrm{GRH})$ for several $L$-functions, we are able to go beyond this barrier for the first time.

Theorem C (see Theorem 5.14). Let $k>2$ be an even integer and $N$ be an odd square-free integer. Fix $F \in S_{k}\left(\Gamma_{0}^{(2)}(N)\right)$. Assume that the refined Gan-Gross-Prasad conjecture [40, equation (1.1)] holds ${ }^{3}$ for Bessel periods of holomorphic cusp forms on $\mathrm{SO}_{5}(\mathbb{A})$. Assume that the GRH holds for L-functions in the Selberg class. ${ }^{4}$ Then we have

$$
|a(F, S)| \ll_{F, \varepsilon} \frac{|\operatorname{disc}(S)|^{\frac{k}{2}-\frac{1}{2}}}{(\log |\operatorname{disc}(S)|)^{\frac{1}{8}-\varepsilon}}
$$

for fundamental matrices $S$.

We note that a bound similar to that obtained in Theorem $\mathrm{C}$ has been recently proved in the special case where $F$ is a Yoshida lift by Blomer and Brumley [5, Corollary 4].

\subsection{The reduction of Theorems $A$ and $B$ to half-integral-weight forms}

The proofs of Theorems A and B rely on reducing these questions to corresponding ones about cusp forms of weight $k-\frac{1}{2}$ on the upper half-plane, exploiting the Fourier-Jacobi expansion of $F$ and the relation between Jacobi forms and classical cusp forms of halfintegral weight. More precisely, using [28] it follows (see §5.2) that the set of primes $p$ such that the $p$ th Fourier-Jacobi coefficient of $F$ is nonzero and has positive density in the set of all primes; fix any $p$ in this set coprime to $N$. Using a classical construction going back to Eichler and Zagier [16, Theorem 5.6] in the case $N=1$ and due to Manickam and Ramakrishnan [41] for square-free $N$, we can now construct a nonzero cusp form $h$ of level $4 N p$ and weight $k-\frac{1}{2}$ whose Fourier coefficients $a(h, n)$ essentially equal some $a(F, S)$ with $|\operatorname{disc}(S)|=n$.

From this construction, Theorem A will follow if we can demonstrate $X^{1-\varepsilon}$ sign changes among the coefficients $a(h, n)$ of the half-integral-weight form $h$ for odd square-free $n \asymp X$ - which is exactly what we prove in Theorem 3.1, a result which builds upon works of Matomäki and Radziwiłł [39, 42] and may be of independent interest. A point worth noting here is that $h$ is not typically a Hecke eigenform (even when $F$ is a Hecke eigenform), as the passage from Siegel cusp forms to Jacobi forms already described is not a functorial correspondence. The main ingredient for our proof of Theorem 3.1 is the demonstration of cancellation in sums of $a(h, n)$ over almost all short intervals, together with bounds on their moments, thereby providing a lower bound on sums of $|a(h, n)|$ over almost all short

${ }^{3}$ A proof of this conjecture has recently been announced by Furusawa and Morimoto (RIMS Conference "Analytic, Geometric and $p$-adic Aspects of Automorphic Forms and $L$-functions," January 2020).

${ }^{4}$ We assume the GRH for Rankin-Selberg $L$-functions $L\left(s, \pi_{1} \times \pi_{2}\right)$ and for symmetric square $L$ functions $L\left(s, \operatorname{Sym}^{2} \pi_{3}\right)$, where for $i=1,2,3, \pi_{i}$ is an automorphic representation of $\mathrm{GL}_{n_{i}}\left(\mathbb{A}_{K}\right)$ with $n_{i} \leq 5$, with $K$ equal to either $\mathbb{Q}$ or an imaginary quadratic field; see also Remark 5.15. 
intervals. Combining the two results shows that over many short intervals, the absolute value of the average of $a(h, n)$ is strictly smaller than that of $|a(h, n)|$. Consequently, a sign change of $a(h, n)$ occurs in many short intervals.

Likewise, Theorem B follows, provided we can demonstrate suitable large values for $|a(h, n)|$. This is done in $\S 4$. The main result of that section, Theorem 4.1 , says that there are at least $X^{1-\varepsilon}$ odd square-free $n \asymp X$ with

$$
|a(h, n)| \geq n^{\frac{k}{2}-\frac{3}{4}} \exp \left(\frac{1}{82} \sqrt{\frac{\log n}{\log \log n}}\right) .
$$

Theorem 4.1 generalizes recent work of Gun, Kohnen, and Soundararajan [21] which dealt with the case of $h$ of level 4. The proof of Theorem 4.1 follows the 'resonance method' strategy of [21, 63]; however, there are additional complications coming from the level which we need to overcome. The starting point of the proof is to use Kohnen's basis for $S_{k+\frac{1}{2}}^{+}(4 N)$ consisting of newforms and an explicit form of Waldspurger's formula to reduce the problem to showing large values for (a weighted average of) a particular central $L$-value, while controlling the sizes of certain other central $L$-values (see Proposition 4.2 and the discussion after it, in particular estimates (31) and (32)). This is achieved by the resonance method as in [21]. A key technical input for this method is the evaluation of the first moment of twisted central $L$-values (Proposition 4.3), which is obtained following the method of [65]. Complications arising from the level show up here in the form of extra congruence and coprimality conditions, and these are dealt with as in [50].

Theorem C, unlike Theorems A and B, does not involve a reduction to half-integralweight forms. We further explain the main ideas behind its proof in $\S 1.4$.

Finally, we remark that a variant of the Fourier-Jacobi expansion trick sketched at the beginning of this subsection has been recently developed by Böcherer and Das to prove the nonvanishing of fundamental Fourier coefficients of Siegel modular forms of degree $n$ [7]. Using their variant, it seems plausible that the methods of this paper may allow one to extend Theorems A and B to Siegel cusp forms of higher degree. We do not pursue this extension here.

\subsection{Central $L$-values for dihedral twists of spin $L$-functions}

For two matrices $S_{1}, S_{2}$ in $\Lambda_{2}$, write $S_{1} \sim S_{2}$ if there exists $A \in \mathrm{SL}_{2}(\mathbb{Z})$ such that $S_{1}=$ ${ }^{t} A S_{2} A$. Set $F \in S_{k}\left(\Gamma_{0}^{(2)}(N)\right)$ with $k>2$ even and $N$ odd and square-free. Using the defining relation for Siegel cusp forms, we see that

$$
a\left(F, S_{1}\right)=a\left(F, S_{2}\right) \quad \text { if } S_{1} \sim S_{2},
$$

thus showing that $a(F, S)$ depends only on the $\mathrm{SL}_{2}(\mathbb{Z})$-equivalence class of the matrix $S$. Let $d<0$ be a fundamental discriminant, let $\mathrm{Cl}_{K}$ denote the ideal class group of $K=\mathbb{Q}(\sqrt{d})$, and let $w(K) \in\{2,4,6\}$ be the number of roots of unity in $K$. It is well known that the $\mathrm{SL}_{2}(\mathbb{Z})$-equivalence classes of matrices in $\Lambda_{2}$ of discriminant $d$ are in natural bijective correspondence with the elements of $\mathrm{Cl}_{K}$. So for any character $\Lambda$ of the 
finite group $\mathrm{Cl}_{K}$, we can define

$$
B(F, \Lambda)=\sum_{S \in \mathrm{Cl}_{K}} a(F, S) \Lambda(S),
$$

which may be viewed as a Bessel period [15, Proposition 3.5].

The space $S_{k}\left(\Gamma_{0}^{(2)}(N)\right)$ has a natural subspace $S_{k}\left(\Gamma_{0}^{(2)}(N)\right)^{\text {CAP }}$ spanned by the SaitoKurokawa lifts. If $F$ is a Saito-Kurokawa lift, then $a(F, S)$ (for fundamental $S$ ) depends only on $d=\operatorname{disc}(S)$ and is fairly well understood. In particular, for $F \in S_{k}\left(\Gamma_{0}^{(2)}(N)\right)^{\mathrm{CAP}}$, the Bessel period $B(F, \Lambda)$ vanishes whenever $\Lambda \neq 1_{K}$, where $1_{K}$ denotes the trivial character of $\mathrm{Cl}_{K}$. Now suppose that $F$ is not a Saito-Kurokawa lift. Let $\phi$ be the adelization of $F$, and suppose that $\phi$ generates an irreducible automorphic representation $\pi$ of $\operatorname{GSp}_{4}(\mathbb{A})$. Böcherer [6] made the remarkable conjecture that $\left|B\left(F, 1_{K}\right)\right|^{2}=A_{F}$. $w(K)^{2} \cdot|d|^{k-1} \cdot L\left(\frac{1}{2}, \pi \otimes \chi_{d}\right)$, where $\chi_{d}$ is the quadratic character associated to $K / \mathbb{Q}$ and $A_{F}$ is a constant depending only on $F$.

More generally, let $\mathcal{A I}(\Lambda)$ be the automorphic representation of $\operatorname{GL}(2, \mathbb{A})$ given by the automorphic induction of $\Lambda$ from $K$; it is generated by (the adelization of the dihedral modular form $\theta_{\Lambda}(z)=\sum_{0 \neq \mathfrak{a} \subset \mathcal{O}_{K}} \Lambda(\mathfrak{a}) e^{2 \pi i N(\mathfrak{a}) z}$ of weight 1 . It is easy to check that $L(s, \pi \otimes$ $\mathcal{A I}(\Lambda))=L(s, \pi) L\left(s, \pi \otimes \chi_{d}\right)$. Now assume that the refined Gan-Gross-Prasad conjecture (see [15, Conjecture 1.12] and [40, (1.1)]) for the pair $(\phi, \Lambda)$ holds true. In fact, this conjecture for $\Lambda=1_{K}$ is now known, thanks to work of Furusawa and Morimoto [18] (which, combined with [15], completes the proof of Böcherer's conjecture), who have also recently announced the proof for general $\Lambda$. Then [15, Theorem 1.13] implies that under some mild assumptions,

$$
|B(F, \Lambda)|^{2}=c_{F} w(K)^{2}|d|^{k-1} L\left(\frac{1}{2}, \pi \times \mathcal{A I}(\Lambda)\right),
$$

where $c_{F}$ is an explicit nonzero constant depending only on $F$ and $L(s, \pi \times \mathcal{A I}(\Lambda))$ is the tensor-product $L$-function of the spin (degree 4) $L$-function of $\pi$ and the standard (degree 2) $L$-function of $\mathcal{A I}(\Lambda)$. We show in Proposition 5.9 that a variant of equation (6) where the equality is replaced by an inequality holds in a more general setup (assuming the refined Gan-Gross-Prasad conjecture).

The identities (5) and (6) demonstrate that the fundamental Fourier coefficients of Hecke eigenforms in $S_{k}\left(\Gamma_{0}^{(2)}(N)\right)$ are intimately connected with central $L$-values of the degree $8 L$-function $L(s, \pi \times \mathcal{A I}(\Lambda))$ as $\Lambda$ varies over the ideal class characters of $K$. By inverting equation (5), we can write

$$
a(F, S)=\frac{1}{\left|\mathrm{Cl}_{K}\right|} \sum_{\Lambda \in \widehat{\mathrm{Cl}}_{K}} B(F, \Lambda) \Lambda^{-1}(S),
$$

which expresses each fundamental $a(F, S)$ as a weighted average of the Bessel periods $B(F, \Lambda)$.

Now, combining equations (6) and (7) with Theorem B, we obtain the following corollaries.

Corollary 1.1. Let $\pi$ be a cuspidal automorphic representation of $\mathrm{GSp}_{4}(\mathbb{A})$ that is not of Saito-Kurokawa type, such that $\pi$ arises from a form in $S_{k}\left(\Gamma_{0}^{(2)}(N)\right)$ with $k>2$ even and 
$N$ odd and square-free. Fix $\varepsilon>0$. Assume the refined Gan-Gross-Prasad conjecture [15, Conjecture 1.12]. Then for all sufficiently large $X$, there are $\geq X^{1-\varepsilon}$ negative fundamental discriminants $d$ with $|d| \asymp X$ such that for $K=\mathbb{Q}(\sqrt{d})$,

$$
\frac{1}{\left|\mathrm{Cl}_{K}\right|} \sum_{\Lambda \in \widehat{\mathrm{Cl}}_{K}} L\left(\frac{1}{2}, \pi \times \mathcal{A I}(\Lambda)\right) \gg_{\pi}|d|^{-1 / 2} \exp \left(\frac{1}{82} \sqrt{\frac{\log |d|}{\log \log |d|}}\right) .
$$

By specializing further to the case of Yoshida lifts, we obtain the following application which is purely about central $L$-values of dihedral twists of classical newforms.

Corollary 1.2. Let $k>2$ be an even integer. Let $N_{1}, N_{2}$ be two positive, square-free integers such that $M=\operatorname{gcd}\left(N_{1}, N_{2}\right)>1$. Let $f$ be a holomorphic newform of weight $2 k-2$ on $\Gamma_{0}\left(N_{1}\right)$ and $g$ be a holomorphic newform of weight 2 on $\Gamma_{0}\left(N_{2}\right)$. Assume that for all primes $p$ dividing $M$, the Atkin-Lehner eigenvalues of $f$ and $g$ coincide. Fix $\varepsilon>0$. Then for all sufficiently large $X$, there are $\geq X^{1-\varepsilon}$ negative fundamental discriminants $d$ with $|d| \asymp X$ with the property that there exists an ideal class group character $\Lambda$ of $K=\mathbb{Q}(\sqrt{d})$ such that

$$
L\left(\frac{1}{2}, f \times \mathcal{A I}(\Lambda)\right) L\left(\frac{1}{2}, g \times \mathcal{A} \mathcal{I}(\Lambda)\right) \gg_{f, g}|d|^{-1 / 2} \exp \left(\frac{1}{82} \sqrt{\frac{\log |d|}{\log \log |d|}}\right) .
$$

Corollary 1.2 strengthens the main theorem of [58], which showed the existence of $\Lambda$ with (simultaneous) nonvanishing for $L\left(\frac{1}{2}, f \times \mathcal{A I}(\Lambda)\right)$ and $L\left(\frac{1}{2}, g \times \mathcal{A I}(\Lambda)\right)$ and remarked, 'While our method gives a lower bound on the number of non-vanishing twists, it does not give a lower bound on the size of the non-vanishing $L$-value itself'. Corollary 1.2 successfully achieves this.

\subsection{Fractional moments of $L$-values}

Combining equations (6) and (7), we can write

$$
|a(F, S)| \ll_{F}|d|^{\frac{k}{2}-\frac{1}{2}} \frac{1}{\left|\mathrm{Cl}_{K}\right|} \sum_{\Lambda \in \widehat{\mathrm{Cl}_{K}}} \sqrt{L\left(\frac{1}{2}, \pi \times \mathcal{A I}(\Lambda)\right)} .
$$

From the foregoing, we see that the generalized Lindelöf hypothesis for $L\left(\frac{1}{2}, \pi \times \mathcal{A I}(\Lambda)\right)$ implies that $|a(F, S)| \ll_{F, \varepsilon}|\operatorname{disc}(S)|^{\frac{k}{2}-\frac{1}{2}+\varepsilon}$, which is still quite far from the bound (3).

Therefore, in order to prove Theorem $\mathrm{C}$, we need to go beyond the bound obtained by a naive application of the generalized Lindelöf hypothesis. We do this by using Soundararajan's method [64] for bounding moments of $L$-functions. Assuming the GRH, we prove the following bound (Theorem 6.1), which, thanks to the estimate (8), implies Theorem C:

$$
\frac{1}{\left|\mathrm{Cl}_{K}\right|} \sum_{\Lambda \in \widehat{\mathrm{Cl}_{K}}} \sqrt{L\left(\frac{1}{2}, \pi \times \mathcal{A I}(\Lambda)\right)} \ll_{\varepsilon} \frac{1}{(\log |d|)^{\frac{1}{8}-\varepsilon}} .
$$

The main contribution to the moments of $L\left(\frac{1}{2}, \pi \times \mathcal{A I}(\Lambda)\right)$ will come from its large values, and we expect that these should be approximated by the large values of 
$\exp \left(\sum_{p^{n}<|d|} \frac{b_{\pi \times \mathcal{A} \mathcal{I}(\Lambda)}\left(p^{n}\right)}{p^{n / 2}}\right)$, where $b_{\pi \times \mathcal{A} \mathcal{I}(\Lambda)}(n)$ is the $n$th coefficient of the Dirichlet series of $\log L(s, \pi \times \mathcal{A I}(\Lambda))$. For ease of discussion, let us assume here that $d$ is prime, $N=1$, and $\pi$ transfers to a cuspidal representation of $\mathrm{GL}_{4} \cdot{ }^{5}$ Separately analyzing the primes, squares of primes, and higher prime powers, we show under GRH that

$$
\sum_{p^{n}<|d|} \frac{b_{\pi \times \mathcal{A} \mathcal{I}(\Lambda)}\left(p^{n}\right)}{p^{n / 2}}=\sum_{p<|d|} \frac{b_{\pi}(p) b_{\mathcal{A I}(\Lambda)}(p)}{\sqrt{p}}-\frac{1}{2} \log \log |d|(1+o(1)),
$$

where $b_{\pi}(p)$ and $b_{\mathcal{A}(\Lambda)}(p)$ respectively denote the $p$ th coefficients of the Dirichlet series of $\log L(s, \pi)$ and of $\log L(s, \mathcal{A I}(\Lambda))$. For primes with $\left(\frac{d}{p}\right)=1$ so that $p \mathcal{O}_{K}=\mathfrak{p} \overline{\mathfrak{p}}$, as $\Lambda$ varies over $\widehat{\mathrm{Cl}_{K}}$, we expect that $b_{\mathcal{A I}(\Lambda)}(p)=\Lambda(\mathfrak{p})+\Lambda(\mathfrak{p})^{-1}$ behaves like the random variable $X_{p}+X_{p}^{-1}$, where $\left\{X_{p}\right\}_{p}$ are independent and identically distributed random variables uniformly distributed on the unit circle - if $\left(\frac{d}{p}\right)=-1, b_{\mathcal{A I}(\Lambda)}(p)=0$. Consequently, the sum on the right-hand side in the foregoing equation is modeled by the random variable $\sum_{p<|d|} \frac{b_{\pi}(p)\left(X_{p}+X_{p}^{-1}\right)}{\sqrt{p}} 1_{\left(\frac{d}{p}\right)=1}$, which can be shown to have a normal limiting distribution as $d \rightarrow \infty$ with mean 0 and variance $2 \sum_{p<|d|} \frac{b_{\pi}(p)^{2}}{p} 1_{\left(\frac{d}{p}\right)=1} \sim \log \log |d|$, which we prove under the GRH. The preceding discussion suggests

$$
\begin{aligned}
\frac{1}{\left|\mathrm{Cl}_{K}\right|} \sum_{\Lambda \in \widehat{\mathrm{Cl}_{K}}} \sqrt{L\left(\frac{1}{2}, \pi \times \mathcal{A I}(\Lambda)\right)} & \asymp(\log |d|)^{-1 / 4} \mathbb{E}\left(\exp \left(\frac{1}{2} \sum_{p<|d|} \frac{b_{\pi}(p)\left(X_{p}+X_{p}^{-1}\right) 1_{\left(\frac{d}{p}\right)=1}}{\sqrt{p}}\right)\right) \\
& \asymp(\log |d|)^{-1 / 8},
\end{aligned}
$$

where in the last step we have used the fact that the moment-generating function of a normal random variable $X$ with mean 0 and variance $\sigma^{2}$ is given by $\mathbb{E}\left(e^{z X}\right)=e^{\frac{1}{2} z^{2} \sigma^{2}}$. Remarkably, Soundararajan's method allows us to make this heuristic argument rigorous for the upper bound, up to the loss of a factor $(\log |d|)^{\varepsilon}$, which occurs due to a suboptimal treatment of the large primes.

\subsection{Notations}

We use the notation $A \ll_{x, y, z} B$ to signify that there exists a positive constant $C$, depending at most upon $x, y, z$, such that $|A| \leq C|B|$. The symbol $\varepsilon$ will denote a small positive quantity. We write $A(x)=O_{y}(B(x))$ if there exists a positive real number $M$ (depending on $y$ ) and a real number $x_{0}$ such that $|A(x)| \leq M|B(x)|$ for all $x \geq x_{0}$.

For a positive integer $n$ with prime factorization $n=\prod_{i=1}^{k} p_{i}^{\alpha_{i}}$, we define $\omega(n)=k$, $\Omega(n)=\sum_{i=1}^{k} \alpha_{i}$. We let $\mu(n)$ denote the Möbius function - that is, $\mu(n)=(-1)^{\omega(n)}$ if $\omega(n)=\Omega(n)$, and $\mu(n)=0$ otherwise. We say that $n$ is square-free if $\mu(n) \neq 0$. We let $(a, b)$ or $\operatorname{gcd}(a, b)$ denote the greatest common divisor of $a$ and $b$.

${ }^{5}$ If this is not the case (e.g., if $\pi$ corresponds to a Yoshida lift), the estimates we give will be slightly different, and the resulting bound for the moment predicted by the heuristic will also differ. 
We say that $d$ is a fundamental discriminant if $d$ is the discriminant of the field $\mathbb{Q}(\sqrt{d})$. For a fundamental discriminant $d$, we let $\chi_{d}$ be the associated quadratic Dirichlet character. Given any representation $\pi$ of a group, we let $\hat{\pi}$ denote the contragredient and $V_{\pi}$ denote the representation space. We use $\mathbb{A}$ to denote the ring of adeles over $\mathbb{Q}$ and we use $\mathbb{A}_{F}$ to denote the ring of adeles over $F$ for a general number field $F$. If $G$ is a reductive group such that the local Langlands correspondence is known for each $G\left(F_{v}\right)$ and $\pi$ is an automorphic representation of $G\left(\mathbb{A}_{F}\right)$, then we formally (as an Euler product over finite places) define the $L$-function $L(s, \rho(\pi)):=L(s, \pi, \rho)$ for each finite-dimensional representation $\rho$ of the dual group. All $L$-functions in this paper will denote the finite part of the $L$-function (i.e., without the Archimedean factors), so that for a number field $F$ and an automorphic representation $\pi$ of $\mathrm{GL}_{n}(F)$, we have $L(s, \pi)=\prod_{v<\infty} L\left(s, \pi_{v}\right)$. All $L$-functions will be normalized to take $s \mapsto 1-s$. For an integer $N$ we denote $L^{N}(s, \pi)=\prod_{v \nmid N} L\left(s, \pi_{v}\right)$. Given a reductive group $G$ and two irreducible automorphic representations $\pi=\otimes_{v} \pi_{v}$ and $\sigma=\otimes_{v} \sigma_{v}$ of $G\left(\mathbb{A}_{F}\right)$, we say that $\pi$ and $\sigma$ are nearly equivalent if $\pi_{v} \simeq \sigma_{v}$ for all but finite many places $v$ of $F$.

\section{Preliminaries on half-integral-weight forms}

The goal of this section is to set up some notation and lay out some key properties concerning cusp forms of half-integral weight on the complex upper half-plane.

\subsection{Notation}

The group $\mathrm{SL}_{2}(\mathbb{R})$ acts on the upper half-plane $\mathbb{H}$ by $\gamma z=\frac{a z+b}{c z+d}$, where $\gamma=\left(\begin{array}{ll}a & b \\ c & d\end{array}\right)$ and $z=x+i y$. For a positive integer $N$, let $\Gamma_{0}(N)$ denote the congruence subgroup consisting of matrices $\left(\begin{array}{ll}a & b \\ c & d\end{array}\right)$ in $\mathrm{SL}_{2}(\mathbb{Z})$ such that $N$ divides $c$. For a complex number $z$, let $e(z)$ denote $e^{2 \pi i z}$.

Let $\theta(z)=\sum_{n=-\infty}^{\infty} e\left(n^{2} z\right)$ be the standard theta function on $\mathbb{H}$. If $A=\left(\begin{array}{ll}a & b \\ c & d\end{array}\right) \in \Gamma_{0}(4)$, we have $\theta(A z)=j(A, z) \theta(z)$, where $j(A, z)$ is the so-called $\theta$-multiplier. For an explicit formula for $j(A, z)$, see $[62,(1.10)]$. Let $S_{k+\frac{1}{2}}(4 N)$ denote the space of holomorphic cusp forms of weight $k+\frac{1}{2}$ for the group $\Gamma_{0}(4 N)$. In other words, a function $f: \mathbb{H} \rightarrow \mathbb{C}$ belongs to $S_{k+\frac{1}{2}}(4 N)$ if

i) $f(A z)=j(A, z)^{2 k+1} f(z)$ for every $A=\left(\begin{array}{ll}a & b \\ c & d\end{array}\right) \in \Gamma_{0}(4 N)$,

ii) $f$ is holomorphic,

iii) and $f$ vanishes at the cusps.

Any $f \in S_{k+\frac{1}{2}}(4 N)$ has the Fourier expansion

$$
f(z)=\sum_{n>0} a(f, n) e(n z) .
$$


We let $c(f, n)$ denote the 'normalized' Fourier coefficients, defined by

$$
c(f, n)=a(f, n) n^{\frac{1}{4}-\frac{k}{2}} .
$$

For $f, g \in S_{k+\frac{1}{2}}(4 N)$, we define the Petersson inner product $\langle f, g\rangle$ by

$$
\langle f, g\rangle=\left[\mathrm{SL}_{2}(\mathbb{Z}): \Gamma_{0}(4 N)\right]^{-1} \int_{\Gamma_{0}(N) \backslash \mathbb{H}} f(z) \overline{g(z)} y^{k+\frac{1}{2}} \frac{d x d y}{y^{2}} .
$$

\subsection{The Kohnen plus space and decomposition into old and newspaces}

Fix positive integers $k, N$ such that $N$ is odd and square-free. We recall the definition of the Kohnen plus space $S_{k+\frac{1}{2}}^{+}(4 N) \subseteq S_{k+\frac{1}{2}}(4 N)$. The space $S_{k+\frac{1}{2}}^{+}(4 N)$ consists of all forms $f$ in $S_{k+\frac{1}{2}}(4 N)$ for which $a(f, n)=0$ whenever $n \equiv(-1)^{k+1}$ or $2 \bmod 4$. According to the results of [33], there exists a canonically defined subspace $S_{k+\frac{1}{2}}^{+, \text {new }}(4 N) \subset S_{k+\frac{1}{2}}^{+}(4 N)$ and a decomposition

$$
S_{k+\frac{1}{2}}^{+}(4 N)=\bigoplus_{\substack{r, \ell \geq 1 \\ r \ell \mid N}} S_{k+\frac{1}{2}}^{+, \text {new }}(4 \ell) \mid U\left(r^{2}\right)
$$

where we define

$$
f \mid U\left(r^{2}\right)=r^{\frac{1}{2}-k} \sum_{n>0} a\left(f, r^{2} n\right) e(n z) .
$$

It is known [62, Proposition 1.5] that if $(r, \ell)=1$, then $U\left(r^{2}\right)$ takes $S_{k+\frac{1}{2}}(4 \ell)$ to $S_{k+\frac{1}{2}}(4 r \ell)$. It is also useful to note that

$$
c\left(f \mid U\left(r^{2}\right), n\right)=c\left(f, r^{2} n\right) .
$$

\subsection{Hecke operators and the Shimura correspondence}

For all primes $p$ coprime to $N$ there exist Hecke operators $T\left(p^{2}\right)$ acting on the space $S_{k+\frac{1}{2}}(4 N)$ (see [62, Theorem 1.7]). A newform in $S_{k+\frac{1}{2}}^{+, \text {new }}(4 N)$ is defined to be an element of $S_{k+\frac{1}{2}}^{+, \text {new }}(4 N)$ that is an eigenfunction of the Hecke operators $T\left(p^{2}\right)$ for $p \nmid N$. The newforms are uniquely determined up to multiplication by nonzero complex numbers and are in fact also eigenforms for the operators $U\left(p^{2}\right)$ for all $p \mid N$ [33, Theorem 2]. The space $S_{k+\frac{1}{2}}^{+, \text {new }}(4 N)$ has an orthogonal basis consisting of newforms.

According to the Shimura lifting [62] as refined by Kohnen in [33], there is an isomorphism

$$
S_{k+\frac{1}{2}}^{+, \text {new }}(4 N) \stackrel{\simeq}{\rightarrow} S_{2 k}^{\text {new }}(N)
$$

as Hecke modules, where $S_{2 k}^{\text {new }}(N)$ is the orthogonal complement of the space of cuspidal oldforms of weight $2 k$ for $\Gamma_{0}(N)$ as defined by Atkin and Lehner [3]. The Shimura lifting takes each newform in $S_{k+\frac{1}{2}}^{+, \text {new }}(4 N)$ (as already defined) to a newform (in the sense of Atkin and Lehner [3, Lemma 18]) in $S_{2 k}^{\text {new }}(N)$ with the same Hecke eigenvalues. More precisely, if $f \in S_{k+\frac{1}{2}}^{+, \text {new }}(4 N)$ is a newform and $g \in S_{2 k}^{\text {new }}(N)$ is the Shimura lift of $f$ according to 
isomorphism (12), then for each prime $p \nmid N$ there exists a real number $\lambda_{f}(p) \in[-2,2]$ (by Deligne's bound for the normalized Hecke eigenvalue) such that

$$
f\left|T\left(p^{2}\right)=\lambda_{f}(p) p^{k-\frac{1}{2}} f, \quad g\right| T(p)=\lambda_{f}(p) p^{k-\frac{1}{2}} g .
$$

In view of decomposition (9) and the fact that the operators $U(p)$ with $p \mid N$ commute with $T\left(p^{2}\right), p \nmid N$, a basis of $S_{k+\frac{1}{2}}^{+}(4 N)$ consisting of eigenforms for $T\left(p^{2}\right), p \nmid N$, is given by

$$
\mathcal{B}_{k+\frac{1}{2}, 4 N}=\bigcup_{\substack{r, \ell \geq 1 \\ r \ell \mid N}}\left\{f \mid U\left(r^{2}\right): f \in \mathcal{B}_{k+\frac{1}{2}, 4 \ell}^{\text {new }}\right\}
$$

where $\mathcal{B}_{k+\frac{1}{2}, 4 \ell}^{\text {new }}$ is an orthogonal basis of $S_{k+\frac{1}{2}}^{+ \text {,new }}(4 \ell)$ consisting of newforms. Note, however, that it is not necessarily the case that all members of $\mathcal{B}_{k+\frac{1}{2}, 4 N}$ are orthogonal to each other. The following result will be useful for us; recall the definitions of $\Omega(n)$ and $\omega(n)$ from $§ 1.5$ :

Lemma 2.1. Let $r, \ell$ be positive, odd, square-free integers with $(r, \ell)=1$ and let $f \in$ $S_{k+\frac{1}{2}}^{+, \text {new }}(4 \ell)$ be a newform. Then for any odd square-free integer $n$, putting $d=(-1)^{k} n$, we have

$$
c\left(f \mid U\left(r^{2}\right), n\right)=c\left(f, r^{2} n\right)=c(f, n) \prod_{p \mid r}\left(\lambda_{f}(p)-\frac{1}{\sqrt{p}}\left(\frac{d}{p}\right)\right) .
$$

Additionally, for any odd integer $r \geq 1$ with $(r, \ell)=1$, we have

$$
\left|c\left(f, r^{2} n\right)\right| \leq 3^{\Omega(r)}|c(f, n)| \text {. }
$$

Proof. The first statement follows from [62, Corollary 1.8(i)]. Using Deligne's bound $\left|\lambda_{f}(p)\right| \leq 2$ and applying [62, Corollary 1.8(ii)], we will establish the second claim by the following simple induction argument. It suffices to show for each $p \nmid 2 \ell$ that

$$
\left|c\left(f, p^{2 m} n\right)\right| \leq 3^{m}|c(f, n)| \text {. }
$$

The case $m=0$ is trivial, and $m=1$ follows from the first claim of the lemma. By [62, Corollary 1.8(ii)], we have, for any $m \geq 1$,

$$
c\left(f, p^{2 m+2} n\right)=\lambda_{f}(p) c\left(f, p^{2 m} n\right)-(-1)^{k\left(p^{2}-1\right) / 2} c\left(f, p^{2 m-2} n\right) .
$$

Hence, for $m \geq 1$ we get

$$
\begin{aligned}
\left|c\left(f, p^{2 m+2} n\right)\right| & \leq 2\left|c\left(f, p^{2 m} n\right)\right|+\left|c\left(f, p^{2 m-2} n\right)\right| \\
& \leq\left(2 \cdot 3^{m}+3^{m-1}\right)|c(f, n)| \leq 3^{m+1}|c(f, n)| .
\end{aligned}
$$

\subsection{An explicit version of Waldspurger's formula}

A well-known formula of Waldspurger [67] that was refined and made explicit in special cases by Kohnen [34] expresses the squares of Fourier coefficients of half-integral-weight 
eigenforms in terms of central $L$-values. We state a version of it here for elements of the basis (13).

Proposition 2.2. Let $r, \ell$ be positive, odd, square-free integers with $(r, \ell)=1$. Let $f$ be a newform in $S_{k+\frac{1}{2}}^{+, \text {new }}(4 \ell)$ and let $g \in S_{2 k}^{\text {new }}(\ell)$ be the Shimura lift of $f$. Then for any squarefree positive integer $n$ with $(n, 4 \ell)=1$, and $d=(-1)^{k} n$, we have

$$
\frac{\left|c\left(f \mid U\left(r^{2}\right), n\right)\right|^{2}}{\langle f, f\rangle}=2^{\omega(\ell)} \frac{L\left(\frac{1}{2}, g \otimes \chi_{d}\right)}{\langle g, g\rangle} \frac{(k-1) !}{\pi^{k}}\left(\prod_{p \mid r}\left(\lambda_{f}(p)-\frac{1}{\sqrt{p}}\left(\frac{d}{p}\right)\right)\right)^{2}
$$

provided that

i) $d \equiv 1(\bmod 4)$ and

ii) for each prime $p \mid \ell$, we have $\left(\frac{d}{p}\right)=w_{p}$, where $w_{p}$ is the eigenvalue for the AtkinLehner operator at $p$ acting on $g$.

If either of these two conditions is not met, then $c\left(f \mid U\left(r^{2}\right), n\right)=0$.

Proof. This follows from [34, Corollary 1], [33, Proposition 4], and Lemma 2.1.

\subsection{Estimates on moments of Fourier coefficients}

Proposition 2.3. Let $f \in S_{k+\frac{1}{2}}^{+}(4 N)$, where $N$ is odd and square-free. Then there exists $M \geq 2$ such that for all sufficiently large $X$,

$$
\sum_{\substack{X \leq n \leq M X \\(n, 2 N)=1}}|c(f, n)|^{2} \mu^{2}(n) \asymp_{f, M} X
$$

and for any $\varepsilon>0$,

$$
\sum_{\substack{X \leq n \leq 2 X \\(n, 2 N)=1}}|c(f, n)|^{4} \mu^{2}(n) \ll_{f, \varepsilon} X^{1+\varepsilon} .
$$

Proof. We first prove the estimate (14). For the upper bound we use the fact that $y^{k+1 / 2}|f(z)|$ is bounded on $\mathbb{H}$, and hence we have

$$
\begin{aligned}
\sum_{\substack{X \leq n \leq 2 X \\
(n, 2 N)=1}}|c(f, n)|^{2} \mu^{2}(n) \leq \sum_{X \leq n \leq 2 X}|c(f, n)|^{2} & \ll \frac{1}{X^{k-1 / 2}} \sum_{n}|c(f, n)|^{2} n^{k-\frac{1}{2}} e^{-4 \pi n / X} \\
& =\frac{1}{X^{k-1 / 2}} \int_{0}^{1}|f(x+i / X)|^{2} d x \ll_{f} X .
\end{aligned}
$$

For the lower bound, we use a result obtained in the proof of [56, Proposition 3.7], which gives, for any $M \geq 1$,

$$
\sum_{(n, 2 N)=1}|c(f, n)|^{2} \mu^{2}(n) e^{-n /(X \sqrt{M})} \gg_{f} X \sqrt{M}
$$


Using the bound (16) along with partial summation, we can bound the tail end of the sum as follows:

$$
\sum_{\substack{n \geq M X \\(n, 2 N)=1}}|c(f, n)|^{2} \mu^{2}(n) e^{-n /(X \sqrt{M})} \ll_{f} M e^{-\sqrt{M}} X .
$$

Combining the two bounds, we have, for $M$ sufficiently large,

$$
\begin{aligned}
\sum_{\substack{n \leq M X \\
(n, 2 N)=1}}|c(f, n)|^{2} \mu^{2}(n) \geq & \sum_{\substack{n \leq M X \\
(n, 2 N)=1}}|c(f, n)|^{2} \mu^{2}(n) e^{-n /(X \sqrt{M})} \\
& =\sum_{\substack{n \geq 1 \\
(n, 2 N)=1}}|c(f, n)|^{2} \mu^{2}(n) e^{-n /(X \sqrt{M})}+O_{f}\left(M e^{-\sqrt{M}} X\right) \gg_{f} X \sqrt{M} .
\end{aligned}
$$

Finally, we note that by the bound (16) the contribution from terms to the left-hand side here with $n \leq X$ is $O_{f}(X)$, which completes the proof of the lower bound in the estimate (14).

For the proof of the bound (15), we use equation (13) to reduce to the case $f=f_{1}$ | $U\left(r^{2}\right)$, where $f_{1} \in S_{k+\frac{1}{2}}^{+, \text {new }}(4 \ell)$ is a newform with $r \ell \mid N$. Using Proposition 2.2, it now suffices to prove that

$$
\sum_{|d| \leq X}^{b} L\left(\frac{1}{2}, g \otimes \chi_{d}\right)^{2} \ll_{g, \varepsilon} X^{1+\varepsilon}
$$

where $g$ is the Shimura lift of $f_{1}$ and the sum is over fundamental discriminants $d$. This follows from the approximate functional equation and Heath-Brown's quadratic large sieve [25], using a straightforward modification of the proof of [25, Theorem 2] (see also [65, Corollary 2.5]).

\section{Sign changes for coefficients of half-integral-weight forms}

\subsection{Statement of main result}

Throughout this section, let $k \geq 2$ be an integer and $N \geq 1$ be odd and square-free. The main theorem to be proved in this section is the following:

Theorem 3.1. Let $f \in S_{k+\frac{1}{2}}^{+}(4 N)$ be a fixed cusp form whose Fourier coefficients $c(f, n)$ are all real. Then there exists $M \geq 2$ such that given any $\varepsilon>0$, the sequence

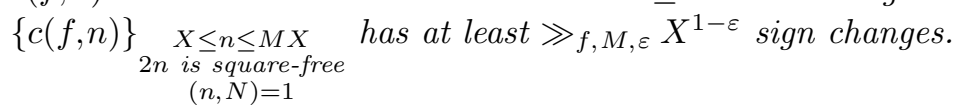

The main novelty here is that this result holds for all cusp forms $f \in S_{k+1 / 2}^{+}(4 N)$, not just Hecke eigenforms, and this is crucial for our later application. Previously it was not apparently even known that there are infinitely many sign changes of $c(f, n)$ as $n$ ranges over square-free integers for $f \in S_{k+\frac{1}{2}}^{+}(4 N)$. 
Our proof builds upon the methods developed in [39,42] and relies upon the following two propositions. The first shows that the size of $|c(f, n)|$ is relatively well behaved for most short intervals $[x, x+y]$.

Proposition 3.2. Let $f \in S_{k+\frac{1}{2}}^{+}(4 N)$. There exists $M \geq 2$ such that given any $\varepsilon>0$ and $2 \leq y \leq X / 2$, there are $\gg_{f, M, \varepsilon} X^{1-\frac{3}{2} \varepsilon}$ integers $X \leq x \leq M X$ such that

$$
\sum_{\substack{x \leq n \leq x+y \\(n, 2 N)=1}}|c(f, n)| \mu^{2}(n)>\frac{y}{X^{\varepsilon}} .
$$

Our other main proposition shows that we can obtain square-root cancelation in sums of $c(f, n)$ over almost all short intervals $[x, x+y]$.

Proposition 3.3. Let $f \in S_{k+\frac{1}{2}}^{+}(4 N)$. Then for $1 \leq y \leq X^{\frac{1}{205}}$, we have

$$
\sum_{X \leq x \leq 2 X}\left|\sum_{\substack{x \leq n \leq x+y \\(n, 2 N)=1}} c(f, n) \mu^{2}(n)\right| \ll_{f} X \sqrt{y}
$$

We will now prove Theorem 3.1 using Propositions 3.2 and 3.3. The proofs of Propositions 3.2 and 3.3 will be given in $\S \S 3.2$ and 3.3 , respectively.

Proof of Theorem 3.1. Observe that if the Fourier coefficients $c(f, n)$ are real and

$$
\left|\sum_{\substack{x \leq n \leq x+y \\(n, 2 N)=1}} c(f, n) \mu^{2}(n)\right|<\sum_{\substack{x \leq n \leq x+y \\(n, 2 N)=1}}|c(f, n)| \mu^{2}(n),
$$

then the interval $[x, x+y]$ must contain a sign change of $c(f, n)$, where $n \in[x, x+y]$ ranges over odd square-free integers that are coprime to $N$. We will show that for most integers $X \leq x \leq M X$, this inequality holds for intervals of length $y=X^{6 \varepsilon}$.

By Chebyshev's inequality, the number of integers $X \leq x \leq M X$ for which

$$
\left|\sum_{\substack{x \leq n \leq x+y \\(n, 2 N)=1}} c(f, n) \mu^{2}(n)\right| \leq \frac{y}{X^{\varepsilon}}
$$

does not hold is

$$
\leq \frac{X^{\varepsilon}}{y} \sum_{X \leq x \leq M X}\left|\sum_{\substack{x \leq n \leq x+y \\(n, 2 N)=1}} c(f, n) \mu^{2}(n)\right| \ll_{f, M} \frac{X^{1+\varepsilon}}{\sqrt{y}}=X^{1-2 \varepsilon}
$$


where we have used Proposition 3.3 in the last inequality. By Proposition 3.2, we have

$$
\sum_{\substack{x \leq n \leq x+y \\(n, 2 N)=1}}|c(f, n)| \mu^{2}(n)>\frac{y}{X^{\varepsilon}}
$$

for all integers $X \leq x \leq M X$ outside an exceptional set of size $\ll_{f, M, \varepsilon} X^{1-3 \varepsilon / 2}$. Hence, there exist at least $\gg_{f, M, \varepsilon} X^{1-3 \varepsilon / 2}$ integers $X \leq x \leq M X$ such that the bounds (17) and (18) hold. Therefore, we obtain at least $\gg_{f, M, \varepsilon} \frac{X^{1-3 \varepsilon / 2}}{y}=X^{1-15 \varepsilon / 2}$ sign changes of $c(f, n)$ along integers $X \leq n \leq M X$ that are odd, square-free, and coprime to $N$.

\subsection{Proof of Proposition 3.2}

We first prove the following result, which is an easy consequence of Proposition 2.3:

Lemma 3.4. Let $f \in S_{k+\frac{1}{2}}^{+}(4 N)$. Then there exists $M \geq 2$ such that given any $\varepsilon>0$,

$$
\sum_{\substack{X \leq n \leq M X \\(n, 2 N)=1 \\|c(f, n)| \leq X^{\varepsilon}}}|c(f, n)| \mu^{2}(n) \gg_{f, M, \varepsilon} X^{1-\varepsilon / 2} .
$$

Proof. Applying Hölder's inequality gives

$$
\sum_{\substack{X \leq n \leq M X \\(n, 2 N)=1}}|c(f, n)|^{2} \mu^{2}(n) \leq\left(\sum_{\substack{X \leq n \leq M X \\(n, 2 N)=1}}|c(f, n)| \mu^{2}(n)\right)^{2 / 3}\left(\sum_{\substack{X \leq n \leq M X \\(n, 2 N)=1}}|c(f, n)|^{4} \mu^{2}(n)\right)^{1 / 3} .
$$

Hence, using Proposition 2.3 we conclude that

$$
\sum_{\substack{X \leq n \leq M X \\(n, 2 N)=1}}|c(f, n)| \mu^{2}(n) \gg_{f, M, \varepsilon} X^{1-\varepsilon / 2} .
$$

Also, by the estimate (14) we have

$$
\sum_{\substack{X \leq n \leq M X \\(n, 2 N)=1 \\|c(f, n)|>X^{\varepsilon}}}|c(f, n)| \mu^{2}(n)<X^{-\varepsilon} \sum_{\substack{X \leq n \leq M X \\(n, 2 N)=1}}|c(f, n)|^{2} \mu^{2}(n) \ll_{f, M, \varepsilon} X^{1-\varepsilon} .
$$

Combining this with the bound (20) completes the proof.

Proof of Proposition 3.2. Let $C(f, n)=|c(f, n)| \mu^{2}(n) 1_{(n, 2 N)=1} 1_{|c(f, n)| \leq X^{\varepsilon}}$. Applying Lemma 3.4, we see that

$$
X^{1-\varepsilon / 2} \ll_{f, M, \varepsilon} \sum_{X+y \leq n \leq 2 M X} C(f, n) \leq \frac{1}{y} \sum_{X \leq x \leq 2 M X} \sum_{x \leq n \leq x+y} C(f, n),
$$

where the second inequality follows because every term in the sum on the left-hand side is counted $\lfloor y\rfloor+1$ times on the right-hand side. Let $S=\left\{X \leq x \leq 2 M X: \sum_{x \leq n \leq x+y} C(f, n)\right.$ $\left.\leq y / X^{\varepsilon}\right\}$. The contribution to the right-hand side from the integers $x \in S$ is 
$\ll_{f, M, \varepsilon} \frac{1}{y} \cdot X \frac{y}{X^{\varepsilon}}=X^{1-\varepsilon}$. Hence we must have

$$
X^{1-\varepsilon / 2} \ll_{f, M, \varepsilon} \frac{1}{y} \sum_{\substack{X \leq x \leq 2 M X \\ x \notin S}} \sum_{x \leq n \leq x+y} C(f, n) \ll_{f, M, \varepsilon} \frac{1}{y} \cdot y X^{\varepsilon} \sum_{\substack{X \leq x \leq 2 M X \\ x \notin S}} 1,
$$

so that $\#\{X \leq x \leq 2 M X: x \notin S\} \gg_{f, M, \varepsilon} X^{1-\frac{3}{2} \varepsilon}$.

\subsection{Proof of Proposition 3.3}

Throughout this section, we write

$$
W(u)=u^{\frac{k-1 / 2}{2}} e^{-2 \pi u} .
$$

The proof of the proposition proceeds directly, beginning with an application of CauchySchwarz. This leads naturally to a shifted convolution sum of Fourier coefficients of $f$ over square-free integers, and to bound this sum we require the following fairly standard result:

Proposition 3.5. Let $f \in S_{k+\frac{1}{2}}^{+}(4 N)$. Then for $1 \leq r \leq X^{\frac{1}{102}}, 0<|h|<X^{\frac{1}{2}}$, and $v \in \mathbb{Z}$ with $(v, r)=1$, we have, for any given $\varepsilon>0$,

$$
\sum_{n \geq 1} c(f, n) c(f, n+h) e\left(\frac{n v}{r}\right) W\left(\frac{n}{X}\right) W\left(\frac{n+h}{X}\right) \ll_{f, \varepsilon} X^{1-\frac{1}{102}+\varepsilon} .
$$

Proof. This is an extension of [39, Proposition 6.1] to the case of general level, and we will describe how to adapt the arguments given there to this case. The initial step is to use the Fourier expansion of $f$ to express the left-hand side of formula (22) as

$$
=\frac{1}{X^{k-\frac{1}{2}}} \int_{0}^{1} f\left(\alpha+\frac{v}{q}+\frac{i}{X}\right) f\left(-\alpha+\frac{i}{X}\right) e(-\alpha h) d \alpha
$$

We now use the circle method following Jutila [30], as in [23, Proposition 2]. An important feature in Jutila's version of the circle method is that we have freedom over our choice of moduli, which we choose as follows:

$$
\mathcal{Q}=\{Q \leq q \leq 2 Q: q=4 N r p \text { and } p \equiv 1(\bmod 4) \text { is prime }\} .
$$

Write $R=\sum_{q \in \mathcal{Q}} \varphi(q)$. Upon applying [23, Proposition 2] with $\delta=Q^{-2+\eta}, Q=X^{1 / 2+2 \eta}$, and $r \leq X^{\eta / 8}$, where $\eta>0$ is chosen later, we get that up to a term of size $O_{f}\left(X^{1-\eta / 8+\varepsilon}\right)$ the integral equals

$$
\frac{Q^{2-\eta}}{2 R X^{k-\frac{1}{2}}} \sum_{q \in \mathcal{Q}} \sum_{d(\bmod q)}^{*} e\left(-\frac{h d}{q}\right) \int_{-\delta}^{\delta} f\left(\frac{d}{q}+\frac{v}{r}+\alpha+\frac{i}{X}\right) f\left(\frac{-d}{q}-\alpha+\frac{i}{X}\right) e(-\alpha h) d \alpha .
$$

Notably, to estimate the error term we use the fact that $y^{\frac{k}{2}+\frac{1}{4}}|f(z)|$ is bounded on $\mathbb{H}$, since $f$ is a cusp form.

Since we have chosen our moduli $q \in \mathcal{Q}$ such that $4 N \mid q$, we are able to use the modularity of $f$ by applying [39, Lemma 6.1], which extends to general level in 
straightforward way, then once again use the Fourier expansion of $f$. Consequently, we have transformed the original sum on the left-hand side of formula (22), which is effectively over $n \leq X^{1+\varepsilon}$, to dual sums which are effectively over $m, n \leq X^{\varepsilon} Q^{2} / X$. The summands in the dual sums include the Fourier coefficients of $f$ twisted by additive characters and factors from the half-integral-weight multiplier system, along with a Kloosterman sum $S(\star,-h ; p)$, where the first argument $\star$ depends on $N, p, m, n, v, r$. An important observation is that since $p$ is a prime with $0<|h|<p$, the Weil bound gives $|S(\star,-h ; p)| \leq 2 \sqrt{p}$ for any $\star \in \mathbb{Z}$. Using the Weil bound and estimating the dual sums over $m, n$ by applying Cauchy-Schwarz and formula (16) to handle the Fourier coefficients of $f$, we can show that expression (23) is bounded by

$$
\ll_{f, \varepsilon} X^{3 / 4+49 \eta / 16+\varepsilon} \text {. }
$$

Recalling our earlier error term of $O\left(X^{1-\eta / 8+\varepsilon}\right)$, which arose from applying Jutila's circle method, we now take $\eta=4 / 51$ to complete the proof.

To sum over square-free integers, we will sieve out integers that have a square divisor and require the following estimate for sums of Fourier coefficients:

Lemma 3.6. Let $f \in S_{k+\frac{1}{2}}^{+}(4 N)$. Then

$$
\sum_{\substack{n \leq X \\(n, 2 N)=1 \\ d^{2} \mid n}}|c(f, n)| \ll_{f, \varepsilon} \frac{X^{1+\varepsilon}}{d^{2}} .
$$

Proof. Just as in the proof of Proposition 2.3, using equation (13) it suffices to consider the case $f=f_{1} \mid U\left(r^{2}\right)$, where $f_{1} \in S_{k+\frac{1}{2}}^{+, \text {new }}(4 \ell)$ is a newform with $r \ell \mid N$. For $(n, 2 N)=1$ write $n=s^{2} m$, where $m$ is odd and square-free. Also, let $t=r s$ and note $(t, \ell)=1$, since $N$ is square-free. Applying Lemma 2.1 we have

$$
c\left(f_{1} \mid U\left(r^{2}\right), n\right)=c\left(f_{1}, t^{2} m\right) \ll_{\varepsilon} t^{\varepsilon}\left|c\left(f_{1}, m\right)\right| .
$$

Using this bound and then applying Cauchy-Schwarz and the bound (16), we get

$$
\sum_{\substack{n \leq X \\(n, 2 N)=1 \\ d^{2} \mid n}}\left|c\left(f_{1} \mid U\left(r^{2}\right), n\right)\right| \ll_{\varepsilon} X^{\varepsilon} \sum_{\substack{t \leq \sqrt{X} \\ d \mid t}} \sum_{m \leq \frac{X}{t^{2}}}\left|c\left(f_{1}, m\right)\right| \ll_{f, \varepsilon} X^{\varepsilon} \sum_{\substack{t \leq \sqrt{X} \\ d \mid t}} \frac{X}{t^{2}} \ll_{\varepsilon} \frac{X^{1+\varepsilon}}{d^{2}} .
$$

Proof of Proposition 3.3. To handle the condition that $2 n$ be square-free, we first recall that the indicator function of square-free numbers is $\mu^{2}(n)=\sum_{d^{2} \mid n} \mu(d)$. We then treat the cases of divisors $d \leq Y$ and $d>Y$ separately, letting

$$
\mu_{\leq Y}^{2}(n)=\sum_{\substack{d^{2} \mid n \\ d \leq Y}} \mu(d), \quad \mu_{>Y}^{2}(n)=\sum_{\substack{d^{2} \mid n \\ d>Y}} \mu(d) .
$$


First we consider the large divisors, and get

$$
\sum_{X \leq x \leq 2 X}\left|\sum_{\substack{x \leq n \leq x+y \\(n, 2 N)=1}} c(f, n) \mu_{>Y}^{2}(n)\right| \leq y \sum_{\substack{n \leq 4 X \\(n, 2 N)=1}}\left|c(f, n) \mu_{>Y}^{2}(n)\right| .
$$

Using the definition of $\mu_{>Y}^{2}(n)$ and applying Lemma 3.6, we see that the right-hand side of this inequality is

$$
\ll y \sum_{d>Y} \sum_{\substack{n \leq 4 X \\ d^{2} \mid n}}|c(f, n)| \ll_{f, \varepsilon} y X^{1+\varepsilon} \sum_{d>Y} \frac{1}{d^{2}} \ll y \frac{X^{1+\varepsilon}}{Y} .
$$

For $Y \geq \sqrt{y} X^{\varepsilon}$, this is $\ll X \sqrt{y}$, as needed.

Next we consider the contribution from the small divisors $d \leq Y$. Let $\widetilde{C}(f, n)=$ $c(f, n) \mu_{\leq Y}^{2}(n) 1_{(n, 2 N)=1}$. Applying Cauchy-Schwarz and using the fact that $W(u)^{2} \gg 1$, for any $u \in[1,2]$ we get

$$
\begin{aligned}
\sum_{X \leq x \leq 2 X}\left|\sum_{x \leq n \leq x+y} \widetilde{C}(f, n)\right| & \leq \sqrt{X}\left(\sum_{X \leq x \leq 2 X}\left|\sum_{x \leq n \leq x+y} \widetilde{C}(f, n)\right|^{2}\right)^{1 / 2} \\
& \ll \sqrt{X}\left(\sum_{x \geq 1}\left|\sum_{x \leq n \leq x+y} \widetilde{C}(f, n)\right|^{2} W\left(\frac{x}{X}\right)^{2}\right)^{1 / 2} .
\end{aligned}
$$

Assume $y \leq X^{1 / 4}$. We use the convention that $c(f, n)=0$ if $n \notin \mathbb{N}$. To estimate the inner sums on the right-hand side, we expand the square, combine appropriate terms, use the fact that $W$ is a smooth function, and apply the bound (16); the right-hand side then equals

$$
\begin{aligned}
& =\sum_{0 \leq h_{1}, h_{2} \leq y} \sum_{n \geq 1} \widetilde{C}\left(f, n+h_{1}\right) \widetilde{C}\left(f, n+h_{2}\right) W\left(\frac{n}{X}\right)^{2} \\
& =\sum_{|h| \leq y} \sum_{n \geq 1} \widetilde{C}(f, n) \widetilde{C}(f, n+h) W\left(\frac{n}{X}\right) W\left(\frac{n+h}{X}\right) \sum_{\substack{0 \leq h_{1}, h_{2} \leq y \\
h_{2}-h_{1}=h}}\left(1+O\left(\frac{y}{n}+\frac{y}{X}\right)\right)+O_{f}(1) \\
& =\sum_{|h| \leq y}(y+1-|h|) \sum_{n \geq 1} \widetilde{C}(f, n) \widetilde{C}(f, n+h) W\left(\frac{n}{X}\right) W\left(\frac{n+h}{X}\right)+O_{f, \varepsilon}\left(y^{3} X^{\varepsilon}\right) .
\end{aligned}
$$

Using the bound (16) once again, we get that the term with $h=0$ in the sum on the right-hand side contributes

$$
\ll y \sum_{n \geq 1}|c(f, n)|^{2} W\left(\frac{n}{X}\right)^{2} \ll_{f, \varepsilon} y X .
$$


We next estimate the contribution from the terms in formula (25) with $h \neq 0$. Recalling the definition of $\mu_{\leq Y}^{2}$ and using the fact that

$$
1_{(n, 2 N)=1}=\sum_{d \mid(n, 2 N)} \mu(d)=\sum_{\substack{d|2 N \\ d| n}} \mu(d),
$$

it follows that the contribution to the right-hand side of formula (25) from the terms with $h \neq 0$ is

$$
\ll y \sum_{\substack{0<|h| \leq y \\ d_{1}, d_{2} \leq Y \\ d_{3}, d_{4} \mid 2 N}} \sum_{\substack{d_{1}^{2}\left|n, d_{2}^{2}\right| n+h \\ d_{3}\left|n, d_{4}\right| n+h}} c(f, n) c(f, n+h) W\left(\frac{n}{X}\right) W\left(\frac{n+h}{X}\right) \mid .
$$

For $n$ with $d_{1}^{2}\left|n, d_{2}^{2}\right| n+h, d_{3} \mid n$, and $d_{4} \mid n+h$, we have $n \equiv a(\bmod r)$ for some $a, r \in \mathbb{Z}$ with $r \leq 16 N^{2} Y^{4}$. Using additive characters to detect this congruence, we get that the inner sum in the previous expression is

$$
\begin{aligned}
& =\sum_{n \equiv a(\bmod r)} c(f, n) c(f, n+h) W\left(\frac{n}{X}\right) W\left(\frac{n+h}{X}\right) \\
& =\frac{1}{r} \sum_{0 \leq v<r} e\left(\frac{-a v}{r}\right) \sum_{n \geq 1} c(f, n) c(f, n+h) e\left(\frac{n v}{r}\right) W\left(\frac{n}{X}\right) W\left(\frac{n+h}{X}\right) .
\end{aligned}
$$

For $v \neq 0$ write $v / r=v^{\prime} / r^{\prime}$, with $\left(r^{\prime}, v^{\prime}\right)=1$, and if $v=0$, set $r^{\prime}=1$. Applying Proposition 3.5 , this sum is $\ll_{f, \varepsilon} X^{1-\frac{1}{102}+\varepsilon}$, provided that $r^{\prime} \leq X^{\frac{1}{102}}$ and $0<|h|<X^{\frac{1}{2}}$. Hence, by this along with the bound (26) we conclude that for $16 N^{2} Y^{4} \leq X^{\frac{1}{102}}$, the right-hand side of formula (25) is $\ll_{f, \varepsilon} y X+y^{2} Y^{2} X^{1-\frac{1}{102}+\varepsilon}+y^{3} X^{\varepsilon}$, which is $\ll y X$, as needed, provided that $y \leq X^{1 / 4}$ and

$$
y Y^{2} X^{\varepsilon} \ll X^{\frac{1}{102}} .
$$

It remains to optimize our parameters. Recall that to handle the contribution of the small divisors, we required $Y \geq \sqrt{y} X^{\varepsilon}$. We now choose $Y=\sqrt{y} X^{\varepsilon}$. Taking the constraint (28) into account, the largest we can choose $y$ is $y=X^{\frac{1}{204}-\frac{3}{2} \varepsilon}$. We conclude by noting that with these choices, we have $16 N^{2} Y^{4} \ll_{N} X^{\frac{1}{102}-3 \varepsilon} \leq X^{\frac{1}{102}}$, as required for the application of Proposition 3.5.

\section{Large values for coefficients of half-integral-weight forms}

The main result of this section, Theorem 4.1, generalizes [21, Theorem 1] (which treated the case $N=1$ ).

\subsection{Statement of main result}

Theorem 4.1. Let $k \geq 2$ be an integer, $N \geq 1$ be odd and square-free, and $h \in S_{k+\frac{1}{2}}^{+}(4 N)$ be a cusp form. Let $\varepsilon>0$ be fixed. Then for all $X$ sufficiently large, there exist at least 
$X^{1-\varepsilon}$ odd square-free integers $n$ coprime to $N$ such that $X \leq n \leq 2 X$ and

$$
|c(h, n)| \geq \exp \left(\frac{1}{82} \sqrt{\frac{\log n}{\log \log n}}\right) .
$$

We will prove this theorem by combining methods of [21] and [39, 50]. Our first job is to reduce the question to bounding central values of $L$-functions. This is done by using the explicit form of Waldspurger's formula due to Kohnen (Proposition 2.2).

\subsection{Reduction to bounds on $L$-values}

Fix an integer $k \geq 2$ and an odd square-free integer $N \geq 1$ throughout $\S 4$. Let $h$ be as in Theorem 4.1. We use the basis (13) to write

$$
h=\sum_{\substack{r, \ell \geq 1 \\ r \ell \mid N}} \sum_{\substack{f \in \mathcal{B}_{k+\frac{1}{2}, 4 \ell}^{\text {new }} \\ k,, \ell, f}} \alpha_{r} \mid U\left(r^{2}\right)
$$

where the coefficients $\alpha_{r, \ell, f}$ depend only on $r, \ell, f, h$. For each odd square-free $n$, we use Lemma 2.1 to get the following identity for the Fourier coefficients:

$$
c(h, n)=\sum_{\ell \mid N} \sum_{f \in \mathcal{B}_{k+\frac{1}{2}, 4 \ell}^{\text {new }}} c(f, n) \sum_{r \mid \frac{N}{\ell}} \alpha_{r, \ell, f} \prod_{p \mid r}\left(\lambda_{f}(p)-\frac{1}{\sqrt{p}}\left(\frac{(-1)^{k} n}{p}\right)\right) .
$$

We already know that $c(h, n) \neq 0$ for some odd square-free $n$ (this follows from [58], for example). So there exist $\ell_{0} \mid N, f_{0} \in \mathcal{B}_{k+\frac{1}{2}, 4 \ell_{0}}^{\text {new }}$, and a reduced residue class $\eta \bmod 4 N$ such that

$$
\begin{array}{r}
\eta \equiv 1(\bmod 4), \quad\left(\frac{\eta}{p}\right)=w_{p} \quad \text { for each } p \mid \ell_{0}, \\
\sum_{r \mid \frac{N}{\ell_{0}}} \alpha_{r, \ell_{0}, f_{0}} \prod_{p \mid r}\left(\lambda_{f_{0}}(p)-\frac{1}{\sqrt{p}}\left(\frac{\eta}{p}\right)\right) \neq 0 .
\end{array}
$$

Here, $w_{p}$ is the eigenvalue of the Atkin-Lehner operator $W_{p}$ acting on $f_{0}$. For brevity, we denote, for each $f \in \mathcal{B}_{k+\frac{1}{2}, 4 \ell}^{\text {new }}$,

$$
\beta_{f}=\sum_{r \mid \frac{N}{\ell}} \alpha_{r, \ell, f} \prod_{p \mid r}\left(\lambda_{f}(p)-\frac{1}{\sqrt{p}}\left(\frac{\eta}{p}\right)\right) .
$$

We will denote the Shimura lift of $f \in \mathcal{B}_{k+\frac{1}{2}, 4 \ell}^{\text {new }}$ by $g_{f} \in S_{2 k}^{\text {new }}(\ell)$ with Fourier coefficients $m^{k-\frac{1}{2}} \lambda_{g_{f}}(m)$ normalized so that $\lambda_{g_{f}}(1)=1$. Also, write $g_{0}$ for $g_{f_{0}}$ and $m^{k-\frac{1}{2}} \lambda_{0}(m)$ for its Fourier coefficients. For each odd, square-free integer $n$ such that $d=(-1)^{k} n \equiv \eta$ $(\bmod 4) N$, we use the triangle inequality, Cauchy-Schwarz, and Proposition 2.2 to obtain 


$$
\begin{aligned}
& |c(h, n)|=\left|\sum_{\ell \mid N} \sum_{\substack{f \in \mathcal{B}^{\text {new }} \\
k+\frac{1}{2}, 4 \ell}} c(f, n) \beta_{f}\right| \\
& \geq\left|\beta_{f_{0}} c_{f_{0}}(n)\right|-\sqrt{\left(\sum_{\ell \mid N} \sum_{f_{0} \neq f \in \mathcal{B}_{k+\frac{1}{2}, 4 \ell}^{\text {new }}}|c(f, n)|^{2}\right)\left(\sum_{\ell \mid N} \sum_{f_{0} \neq f \in \mathcal{B}_{k+\frac{1}{2}, 4 \ell}^{\text {new }}}\left|\beta_{f}\right|^{2}\right)}
\end{aligned}
$$

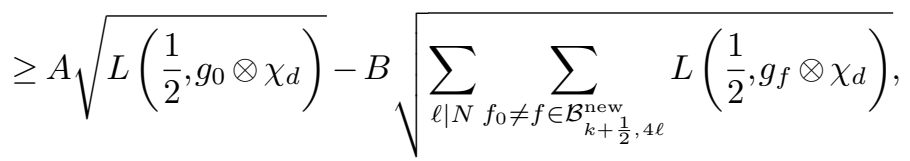

where $A>0$ and $B>0$ are independent of $d$. Now Theorem 4.1 follows from the following auxiliary result:

Proposition 4.2. Let $C \geq 0$ be a constant, $\varepsilon>0$, and let $\eta(\bmod 4 N)$ be a fixed reduced residue class with $\eta \equiv 1(\bmod 4)$. Given $\ell_{0} \mid N$, let $f_{0} \in \mathcal{B}_{k+\frac{1}{2}, 4 \ell_{0}}^{\text {new }}$ be a newform as before with Shimura lift $g_{0}$. For sufficiently large $X$, there are $\geq X^{1-\varepsilon}$ odd square-free integers $n \in[X, 2 X]$, such that $d=(-1)^{k} n \equiv \eta(\bmod 4 N)$ and

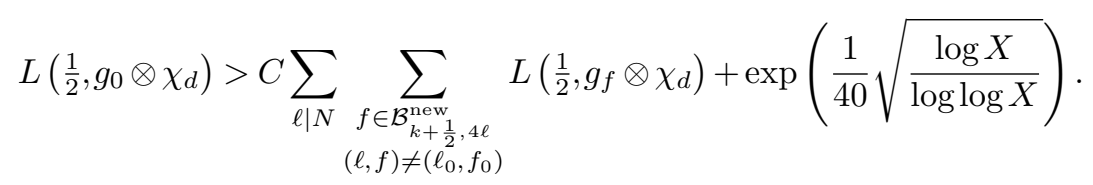

We first explain how Proposition 4.2 implies Theorem 4.1. Put $L_{0}=L\left(\frac{1}{2}, g_{0} \otimes \chi_{d}\right)$ and $L_{1}=\sum_{\ell \mid N} \sum_{f_{0} \neq f \in \mathcal{B}_{k+\frac{1}{2}, 4 \ell}^{\text {new }}} L\left(\frac{1}{2}, g_{f} \otimes \chi_{d}\right)$. Put $C=2 B^{2} / A^{2}$. Combining Proposition 4.2 and the estimate (29),

$$
\begin{aligned}
|c(h, n)| & \geq A \sqrt{L_{0}}-B \sqrt{L_{1}}>A \sqrt{C L_{1}+\exp \left(\frac{1}{40} \sqrt{\frac{\log X}{\log \log X}}\right)}-B \sqrt{L_{1}} \\
& \geq \frac{A}{\sqrt{2}}\left(\sqrt{C L_{1}}+\exp \left(\frac{1}{80} \sqrt{\left.\frac{\log X}{\log \log X}\right)}\right)-B \sqrt{L_{1}}\right. \\
& =\frac{A}{\sqrt{2}} \exp \left(\frac{1}{80} \sqrt{\frac{\log X}{\log \log X}}\right) \geq \exp \left(\frac{1}{82} \sqrt{\frac{\log n}{\log \log n}}\right)
\end{aligned}
$$

for sufficiently large $X$. We now proceed with the proof of Proposition 4.2. For $\eta \equiv 1$ $(\bmod 4)$, let

$$
\mathcal{D}_{N, \eta}=\left\{d=(-1)^{k} n: \mu^{2}(2 n) \neq 0,(n, N)=1, d \equiv \eta(\bmod 4 N)\right\}
$$

and

$$
\mathcal{D}_{N, \eta}(X)=\mathcal{D}_{N, \eta} \cap[X, 2 X]
$$


For each such $d$ we introduce a resonance polynomial

$$
R(d)=\sum_{m \leq M} r(m) \lambda_{0}(m) \chi_{d}(m),
$$

where the coefficients $r(m)$ are multiplicative and supported on square-free integers. At primes we set

$$
r(p)= \begin{cases}\frac{L}{\sqrt{p} \log p} & \text { if } L^{2} \leq p \leq L^{4} \text { and } p \nmid N \\ 0 & \text { otherwise, }\end{cases}
$$

where $L=\frac{1}{8} \sqrt{\log M \log \log M}$, with $M=X^{1 / 24}$.

The strategy to prove the estimate (30) is to consider the quantity

$$
\sum_{d \in \mathcal{D}_{N, \eta}(X)} L\left(\frac{1}{2}, g_{0} \otimes \chi_{d}\right)|R(d)|^{2} .
$$

Let $\mathcal{S}$ be the subset of $\mathcal{D}_{N, \eta}(X)$ for which the estimate (30) holds. Then certainly

$$
\begin{aligned}
& \sum_{d \in \mathcal{D}_{N, \eta}(X)} L\left(\frac{1}{2}, g_{0} \otimes \chi_{d}\right)|R(d)|^{2} \\
& \leq \sum_{d \in \mathcal{D}_{N, \eta}(X)}\left(\sum_{\substack{\ell=1 \\
\ell \mid N}}^{\infty} \sum_{\substack{f \in \mathcal{B}^{\text {new }} \\
(\ell, f) \neq\left(\frac{1}{2}, 4 \ell\right.}} C L\left(\frac{1}{2}, g_{f} \otimes \chi_{d}\right)+\exp \left(\frac{1}{40} \sqrt{\frac{\log X}{\log \log X}}\right)\right)|R(d)|^{2} \\
& \quad+\sum_{d \in \mathcal{S}} L\left(\frac{1}{2}, g_{0} \otimes \chi_{d}\right)|R(d)|^{2} .
\end{aligned}
$$

Suppose that the following estimates hold:

$$
\begin{gathered}
\sum_{d \in \mathcal{D}_{N, \eta}(X)} L\left(\frac{1}{2}, g_{0} \otimes \chi_{d}\right)|R(d)|^{2} \gg_{k, N} X \cdot \mathcal{R} \cdot \exp \left(\left(\frac{1}{2}+o(1)\right) \frac{L}{\log L}\right) \\
\sum_{d \in \mathcal{D}_{N, \eta}(X)} L\left(\frac{1}{2}, g_{f} \otimes \chi_{d}\right)|R(d)|^{2} \ll_{k, N} X \cdot \mathcal{R} \cdot \exp \left(o\left(\frac{L}{\log L}\right)\right) \quad \text { for } f \neq f_{0}, \\
\sum_{d \in \mathcal{D}_{N, \eta}(X)}|R(d)|^{2} \leq \frac{2 X}{\pi^{2}} \cdot \mathcal{R}+O(X) \\
\sum_{d \in \mathcal{D}_{N, \eta}(X)}|R(d)|^{6} \ll X \cdot \exp \left(O\left(\frac{\log X}{\log \log X}\right)\right)
\end{gathered}
$$

where

$$
\mathcal{R}=\prod_{L^{2} \leq p \leq L^{4}}\left(1+r(p)^{2} \lambda_{0}(p)^{2}\right) .
$$


Assuming the estimates (31)-(34), the proof of Proposition 4.2 can be finished as follows. We observe that

$$
\begin{aligned}
X \cdot \mathcal{R} \cdot \exp \left(\left(\frac{1}{2}+o(1)\right) \frac{L}{\log L}\right) & \ll_{k, N} \sum_{d \in \mathcal{D}_{N, \eta}(X)} L\left(\frac{1}{2}, g_{0} \otimes \chi_{d}\right)|R(d)|^{2} \\
& \ll_{k, N} X \cdot \mathcal{R} \cdot \exp \left(o\left(\frac{L}{\log L}\right)\right)+\sum_{d \in \mathcal{S}} L\left(\frac{1}{2}, g_{0} \otimes \chi_{d}\right)|R(d)|^{2}
\end{aligned}
$$

by using the estimates (31)-(33). Hence

$$
X \cdot \mathcal{R} \cdot \exp \left(\left(\frac{1}{2}+o(1)\right) \frac{L}{\log L}\right) \ll_{k, N} \sum_{d \in \mathcal{S}} L\left(\frac{1}{2}, g_{0} \otimes \chi_{d}\right)|R(d)|^{2} .
$$

On the other hand, the right-hand side can be estimated by Hölder's inequality and the estimate (34) as

$$
\begin{aligned}
& \leq\left(\sum_{d \in \mathcal{D}_{N, \eta}(X)} L\left(\frac{1}{2}, g_{0} \otimes \chi_{d}\right)^{2}\right)^{1 / 2} \cdot|\mathcal{S}|^{1 / 6}\left(\sum_{d \in \mathcal{S}}|R(d)|^{6}\right)^{1 / 3} \\
& \ll_{k, N} X^{1 / 2+\varepsilon / 2}|\mathcal{S}|^{1 / 6} \cdot\left(X \cdot \exp \left(O\left(\frac{\log X}{\log \log X}\right)\right)\right)^{1 / 3},
\end{aligned}
$$

where, as before, the average of the squares of central $L$-values is estimated by using the quadratic large sieve of Heath-Brown [25] (here we can extend the sum to all fundamental discriminants $\leq X$ in magnitude by nonnegativity). Combining this with the estimate (35) gives $|\mathcal{S}| \gg_{k, N} X^{1-\varepsilon / 2} \geq X^{1-\varepsilon}$, as desired.

So it suffices to establish estimates (31)-(34). Notice that the estimates (33) and (34) follow directly from [21, Proposition 3] by simply estimating

$$
\begin{gathered}
\sum_{d \in \mathcal{D}_{N, \eta}(X)}|R(d)|^{2} \leq \sum_{\substack{X \leq(-1)^{k} d \leq 2 X \\
d \equiv 1(\bmod 4)}}|R(d)|^{2} \quad \text { and } \\
\sum_{d \in \mathcal{D}_{N, \eta}(X)}|R(d)|^{6} \leq \sum_{\substack{X \leq(-1)^{k} d \leq 2 X \\
d \equiv 1(\bmod 4)}}|R(d)|^{6} .
\end{gathered}
$$

The other two estimates are consequences of the following first moment result.

Proposition 4.3. Let $N \geq 1$ be a positive integer and let $g \in S_{2 k}^{\text {new }}(\ell)$ for some $\ell \mid N$. Suppose that $u$ is an odd positive integer coprime to $N$ and write $u=u_{1} u_{2}^{2}$, with $u_{1}$ square-free. Let $\Phi$ denote a smooth and compactly supported function in $[1 / 2,5 / 2]$. Then

$$
\begin{aligned}
& \sum_{d \in \mathcal{D}_{N, \eta}} L\left(\frac{1}{2}, g \otimes \chi_{d}\right) \chi_{d}(u) \Phi\left(\frac{|d|}{X}\right) \\
& \quad=\frac{X \lambda_{g}\left(u_{1}\right)}{2 N u_{1}^{1 / 2}}\left(\int_{0}^{\infty} \Phi(\xi) \mathrm{d} \xi\right) L_{g, \eta}\left(\frac{1}{2}\right) L\left(1, \operatorname{Sym}^{2} g\right) \mathcal{G}(1 ; u)+O_{k, N, \Phi, \varepsilon}\left(X^{7 / 8+\varepsilon} u^{3 / 8+\varepsilon}\right) .
\end{aligned}
$$


Here $L_{g, \eta}(1 / 2) \neq 0$ is the Dirichlet series given in equation (36) and $\mathcal{G}(1 ; u)$ is the Euler product defined in equation (41). Furthermore, $\mathcal{G}(1 ; \cdot)$ is a multiplicative function satisfying $\mathcal{G}\left(1 ; p^{k}\right)=1+O(1 / p)$ at prime powers.

\subsection{A twisted first moment asymptotic}

In this subsection, we sketch the proof of Proposition 4.3. The starting point is the approximate functional equation (which follows by an easy modification of the proof of [50, Lemma 5]) saying that

$$
L\left(\frac{1}{2}, g \otimes \chi_{d}\right)=2 \sum_{\substack{m=1 \\(m, 2 N)=1}}^{\infty} \frac{\lambda_{g}(m) \chi_{d}(m)}{\sqrt{m}} W_{g, \eta}\left(\frac{m}{|d|}\right)
$$

where $W_{g, \eta}$ is a smooth weight function defined by, for $c>1 / 2$,

$$
W_{g, \eta}(\xi)=\frac{1}{2 \pi i} \int_{(c)} L_{g, \eta}\left(s+\frac{1}{2}\right) \frac{\Gamma(s+k)}{\Gamma(k)}\left(\frac{\sqrt{\ell}}{2 \pi \xi}\right)^{s} \frac{\mathrm{d} s}{s}, \quad L_{g, \eta}(s)=\sum_{\substack{m=1 \\ p|m| 2 N}}^{\infty} \frac{\lambda_{g}(m) \chi_{d}(m)}{m^{s}} .
$$

Notice that the value of $L_{g, \eta}(s)$ is the same for each $d \equiv \eta(\bmod 4 N)$. The weight function satisfies $W_{g, \eta}(\xi)=L_{g, \eta}(1 / 2)+O_{k, N, \varepsilon}\left(\xi^{\frac{1}{2}-\varepsilon}\right)$ as $\xi \longrightarrow 0$ and $W_{g, \eta}(\xi) \ll_{k, N, A}|\xi|^{-A}$ for any $A \geq 1$ as $\xi \longrightarrow \infty$. Thus the sum we have to evaluate takes the shape

$$
2 \sum_{\substack{m=1 \\(m, 2 N)=1}}^{\infty} \frac{\lambda_{g}(m)}{\sqrt{m}} \sum_{d \in \mathcal{D}_{N, \eta}} \chi_{d}(m u) W_{g, \eta}\left(\frac{m}{|d|}\right) \Phi\left(\frac{|d|}{X}\right) .
$$

Notice that by definition, any $d \in \mathcal{D}_{N, \eta}$ is square-free and odd. We pick out this property by the identity

$$
\sum_{\substack{\alpha=1 \\(\alpha, 2 N)=1 \\ \alpha^{2} \mid d}}^{\infty} \mu(\alpha)= \begin{cases}1 & \text { if } d \text { is square-free } \\ 0 & \text { otherwise }\end{cases}
$$

Note that this identity holds without the condition $(\alpha, 2 N)=1$, but this can be added, as by construction $(d, 2 N)=1$ for $d \in \mathcal{D}_{N, \eta}$. Inserting this into the expression (37) that the $d$-sum is given by

$$
\sum_{\substack{\alpha=1 \\(\alpha, 2 N u)=1}}^{\infty} \mu(\alpha)\left(\frac{\alpha^{2}}{m u}\right) \sum_{r \equiv \eta \overline{\alpha^{2}}(\bmod 4 N)}\left(\frac{r}{m u}\right) W_{g, \eta}\left(\frac{m}{r \alpha^{2}}\right) \Phi\left(\frac{r \alpha^{2}}{X}\right) .
$$

We will evaluate the $r$-sum by applying a version of the Poisson summation formula [50, Lemma 7]. The terms where $m u$ is a square will contribute the main term in the zero-frequency term on the dual side, and the rest will give the error term. Using standard arguments [39], the contribution of the latter terms can be bounded by $\ll_{k, N, \Phi, \varepsilon} X^{7 / 8+\varepsilon} u^{3 / 8+\varepsilon}$. 
Using equation (36), the zero-frequency contribution is given by

$$
\begin{aligned}
& \frac{X}{2 N} \cdot \frac{1}{2 \pi i} \int_{(c)}\left(\int_{0}^{\infty} \Phi(\xi) \xi^{s} \mathrm{~d} \xi\right) L_{g, \eta}\left(s+\frac{1}{2}\right) \frac{\Gamma(s+k)}{\Gamma(k)}\left(\frac{\sqrt{\ell} X}{2 \pi}\right)^{s} \\
& \quad \times \sum_{\substack{\alpha=1 \\
(\alpha, 2 N u)=1}}^{\infty} \frac{\mu(\alpha)}{\alpha^{2}} \sum_{\substack{m=1 \\
(m, 2 N \alpha)=1 \\
m u \text { is a square }}}^{\infty} \frac{\lambda_{g}(m)}{m^{s+1 / 2}} \frac{\varphi(m u)}{m u} \frac{\mathrm{d} s}{s} .
\end{aligned}
$$

A simple computation shows that for $(m, 2 N)=1$,

$$
\sum_{\substack{\alpha=1 \\(\alpha, 2 N u m)=1}}^{\infty} \frac{\mu(\alpha)}{\alpha^{2}} \cdot \frac{\varphi(m u)}{m u}=\prod_{p \nmid 2 N}\left(1-\frac{1}{p^{2}}\right) \prod_{p \mid u m}\left(1+\frac{1}{p}\right)^{-1},
$$

leading to

$$
\begin{aligned}
& \sum_{\substack{\alpha=1 \\
(\alpha, 2 N u)=1}}^{\infty} \frac{\mu(\alpha)}{\alpha^{2}} \sum_{\substack{m=1 \\
(m, 2 N \alpha)=1 \\
m u \text { is a square }}}^{\infty} \frac{\lambda_{g}(m)}{m^{s+1 / 2}} \cdot \frac{\varphi(m u)}{m u} \\
& =\prod_{p \nmid 2 N}\left(1-\frac{1}{p^{2}}\right) \sum_{\substack{m=1 \\
(m, 2 N)=1 \\
m u \text { is a square }}}^{\infty} \frac{\lambda_{g}(m)}{m^{s+1 / 2}} \prod_{p \mid u m}\left(1+\frac{1}{p}\right)^{-1} .
\end{aligned}
$$

For $m u$ a square and $u=u_{1} u_{2}^{2}$ with $u_{1}$ square-free, it follows that $m=u_{1} \ell^{2}$ for some $\ell \in \mathbb{Z}$. Hence the right-hand side of the previous display is

$$
\begin{aligned}
& \prod_{p \nmid 2 N}\left(1-\frac{1}{p^{2}}\right) \sum_{\substack{\ell=1 \\
\ell, 2 N)=1}}^{\infty} \frac{\lambda_{g}\left(u_{1} \ell^{2}\right)}{u_{1}^{s+1 / 2} \ell^{2 s+1}} \prod_{p \mid u \ell}\left(1+\frac{1}{p}\right)^{-1} \\
= & \frac{1}{u_{1}^{s+1 / 2}} \prod_{p \nmid 2 N}\left(1-\frac{1}{p^{2}}\right) \prod_{p \nmid 2 N u}\left(1+\sum_{k=1}^{\infty} \frac{\lambda_{g}\left(p^{2 k}\right)}{p^{k(2 s+1)}}\left(1+\frac{1}{p}\right)^{-1}\right) \\
& \times \prod_{p \mid u}\left(\sum_{k=0}^{\infty} \frac{\lambda_{g}\left(p^{2 k+\operatorname{ord}_{p}\left(u_{1}\right)}\right)}{p^{k(2 s+1)}}\right)\left(1+\frac{1}{p}\right)^{-1} \\
= & \frac{1}{u_{1}^{s+1 / 2}} L\left(2 s+1, \operatorname{Sym}^{2} g\right) \prod_{p \mid 2 N} L_{p}\left(2 s+1, \operatorname{Sym}^{2} h\right)^{-1} \prod_{p \mid u}\left(1-\frac{1}{p}\right)\left(\sum_{k=0}^{\infty} \frac{\lambda_{g}\left(p^{2 k+\operatorname{ord}_{p}\left(u_{1}\right)}\right)}{p^{k(2 s+1)}}\right) \\
& \cdot L_{p}\left(2 s+1, \operatorname{Sym}^{2} g\right)^{-1} \prod_{p \nmid 2 N u}\left(1-\frac{1}{p^{2}}\right)\left(1+\sum_{k=1}^{\infty} \frac{\lambda_{g}\left(p^{2 k}\right)}{p^{k(2 s+1)}}\left(1+\frac{1}{p}\right)^{-1}\right) L_{p}\left(2 s+1, \operatorname{Sym}^{2} g\right)^{-1} .
\end{aligned}
$$


From this it is easy to see, by using the Euler product expression of the symmetric square $L$-function, that for $p \nmid 2 N u$ the corresponding Euler factor is

$$
=\left(1-\frac{1}{p}\right)\left(1-\frac{1}{p^{2 s+1}}\right)\left(\frac{1}{p}\left(1-\frac{\alpha_{p}^{2}}{p^{2 s+1}}\right)\left(1-\frac{\beta_{p}^{2}}{p^{2 s+1}}\right)+1+\frac{1}{p^{2 s+1}}\right),
$$

where $\left\{\alpha_{p}, \beta_{p}\right\}$ are the Satake parameters of the cusp form $g$ at $p$.

Similarly, for $p \mid u, p \nmid u_{1}$, the corresponding Euler factor is $\left(1-\frac{1}{p}\right)\left(1-\frac{1}{p^{4 s+2}}\right)$. For $p \mid u_{1}$, the corresponding Euler factor is $\left(1-\frac{1}{p}\right)\left(1-\frac{1}{p^{2 s+1}}\right) \lambda_{g}(p)$ by using the relations $\lambda_{g}\left(p^{j}\right)=\left(\alpha_{p}^{j+1}-\beta_{p}^{j+1}\right) /\left(\alpha_{p}-\beta_{p}\right)$ for $j \geq 0$ and $\alpha_{p} \beta_{p}=1$. And finally, for $p \mid 2 N$ the corresponding Euler factor is clearly $L_{p}\left(2 s+1, \operatorname{Sym}^{2} g\right)^{-1}$.

To summarize, the right-hand side of equation (40) equals

$$
\frac{\lambda_{g}\left(u_{1}\right)}{u_{1}^{s+1 / 2}} L\left(2 s+1, \operatorname{Sym}^{2} g\right) \mathcal{G}(2 s+1 ; u)
$$

where $\mathcal{G}(2 s+1 ; u)=\prod_{p} \mathcal{G}_{p}(2 s+1 ; u)$ is the Euler product locally given by

$$
\begin{aligned}
& \mathcal{G}_{p}(2 s+1 ; u) \\
& \quad= \begin{cases}L_{p}\left(2 s+1, \text { Sym }^{2} g\right)^{-1} & \text { if } p \mid 2 N, \\
\left(1-\frac{1}{p}\right)\left(1-\frac{1}{p^{2 s+1}}\right) & \text { if } p \mid u_{1}, \\
\left.1-\frac{1}{p}\right)\left(1-\frac{1}{p^{4 s+2}}\right) & \text { if } p \mid u_{2}, p \nmid u_{1}, \\
\left(1-\frac{1}{p}\right)\left(1-\frac{1}{p^{2 s+1}}\right)\left(1+\frac{1}{p}\left(1-\frac{\alpha_{p}^{2}}{p^{2 s+1}}\right)\left(1-\frac{\beta_{p}^{2}}{p^{2 s+1}}\right)+\frac{1}{p^{2 s+1}}\right) & \text { if } p \nmid 2 N u .\end{cases}
\end{aligned}
$$

By estimating trivially, it follows that $\mathcal{G}(2 s+1 ; u)$ extends analytically to the domain $\operatorname{Re}(s)>-1 / 4$ and is bounded there by

$$
\ll \prod_{p \nmid 2 N u}\left(1+\frac{O(1)}{\sqrt{p}}\right) \ll_{N, \varepsilon} u^{\varepsilon} .
$$

Consequently, the $s$-integrand in the expression (39) extends to an analytic function the domain $\operatorname{Re}(s)>-1 / 4$ (apart from a simple pole at $s=0$ ). Thus moving the line of integration in formula (39) to the line $\operatorname{Re}(s)=-1 / 4+\varepsilon$ shows that the expression equals

$$
\frac{X \lambda_{g}\left(u_{1}\right)}{2 N u_{1}^{1 / 2}}\left(\int_{0}^{\infty} \Phi(\xi) \mathrm{d} \xi\right) L_{g, \eta}\left(\frac{1}{2}\right) L\left(1, \operatorname{Sym}^{2} g\right) \mathcal{G}(1 ; u)+O_{k, N, \Phi, \varepsilon}\left(X^{3 / 4+\varepsilon}\right),
$$

where the main term comes from the residue at $s=0$ and the error term from the contour shift. It follows immediately from the definition of $\mathcal{G}(s ; u)$ that $\mathcal{G}(1 ; \cdot)$ is multiplicative and that $\mathcal{G}\left(1 ; p^{k}\right)=1+O(1 / p)$ at prime powers. This concludes the sketch of the proof of Proposition 4.3. 


\subsection{Proofs of the estimates}

We are now ready to prove the estimates (31) and (32). As the arguments are similar to [21], we will be brief. Let us denote

$$
A_{g, \eta}(\Phi)=\frac{1}{2 N}\left(\int_{0}^{\infty} \Phi(\xi) \mathrm{d} \xi\right) L_{g, \eta}\left(\frac{1}{2}\right) L\left(1, \operatorname{Sym}^{2} g\right)
$$

and

$$
B_{g}(u)=\frac{\lambda_{g}\left(u_{1}\right)}{u_{1}^{1 / 2}} \mathcal{G}(1 ; u)
$$

for $u=u_{1} u_{2}^{2}$ with $u_{1}$ square-free.

Let $\Phi$ be a compactly supported smooth weight function. Our aim is now to evaluate

$$
\sum_{d \in \mathcal{D}_{N, \eta}} L\left(\frac{1}{2}, g \otimes \chi_{d}\right)|R(d)|^{2} \Phi\left(\frac{|d|}{X}\right)
$$

for $g=g_{f} \in S_{2 k}^{\text {new }}(\ell)$, where $f \in \mathcal{B}_{k+\frac{1}{2}, 4 \ell}^{\text {new }}$ with $\ell \mid N$, by choosing $\Phi$ appropriately for given $f$.

By opening the definition of $R(d)$ and using Proposition 4.3, the sum (42) equals

$$
\begin{aligned}
\sum_{d \in \mathcal{D}_{N, \eta}} L\left(\frac{1}{2}, g \otimes \chi_{d}\right) \Phi\left(\frac{|d|}{X}\right) \sum_{n_{1}, n_{2} \leq M} r\left(n_{1}\right) r\left(n_{2}\right) \lambda_{0}\left(n_{1}\right) \lambda_{0}\left(n_{2}\right) \chi_{d}\left(n_{1} n_{2}\right) \\
=X \cdot A_{g, \eta}(\Phi) \sum_{n_{1}, n_{2} \leq M} r\left(n_{1}\right) r\left(n_{2}\right) \lambda_{0}\left(n_{1}\right) \lambda_{0}\left(n_{2}\right) B_{g}\left(n_{1} n_{2}\right) \\
\quad+O_{k, N, \Phi, \varepsilon}\left(X^{7 / 8+\varepsilon} \sum_{n_{1}, n_{2} \leq M} r\left(n_{1}\right) r\left(n_{2}\right)\left|\lambda_{0}\left(n_{1}\right) \lambda_{0}\left(n_{2}\right)\right|\left(n_{1} n_{2}\right)^{3 / 8+\varepsilon}\right)
\end{aligned}
$$

as $r(n)$ vanishes, unless $(n, N)=1$. Using Deligne's bound, we obtain as in [21, §6] that the error term is $\ll_{k, N, \Phi} X^{99 / 100}$.

By making use of the fact that

$$
n_{1} n_{2}=\frac{n_{1} n_{2}}{\left(n_{1}, n_{2}\right)^{2}} \cdot\left(n_{1}, n_{2}\right)^{2},
$$

with the first factor on the right-hand side square-free in our case, as the function $r(n)$ is supported only on square-free integers, the main term can be written as

$$
X \cdot A_{g, \eta}(\Phi) \sum_{n_{1}, n_{2} \leq M} r\left(n_{1}\right) r\left(n_{2}\right) \lambda_{0}\left(n_{1}\right) \lambda_{0}\left(n_{2}\right) \frac{\lambda_{g}\left(\frac{n_{1} n_{2}}{\left(n_{1}, n_{2}\right)^{2}}\right)}{\left(\frac{n_{1} n_{2}}{\left(n_{1}, n_{2}\right)^{2}}\right)^{1 / 2}} \cdot \mathcal{G}\left(1 ; n_{1} n_{2}\right) .
$$

Our aim is to use multiplicativity, and so we need to extend the sum over all integers. To do so we must show that the terms with $\max \left(n_{1}, n_{2}\right)>M$ can be added with a tolerable 
error. Using Rankin's trick, these terms contribute

$$
\begin{aligned}
& \ll_{k, N} X \sum_{\max \left(n_{1}, n_{2}\right)>M} r\left(n_{1}\right) r\left(n_{2}\right)\left|\lambda_{0}\left(n_{1}\right) \lambda_{0}\left(n_{2}\right)\right| \cdot\left|\mathcal{G}\left(1 ; n_{1} n_{2}\right)\right| \cdot\left|\frac{\lambda_{g}\left(\frac{n_{1} n_{2}}{\left(n_{1}, n_{2}\right)^{2}}\right)}{\left(\frac{n_{1} n_{2}}{\left(n_{1}, n_{2}\right)^{2}}\right)^{1 / 2}}\right| \\
& \ll_{k, N} X \sum_{n_{1}, n_{2}=1}^{\infty} r\left(n_{1}\right) r\left(n_{2}\right)\left|\lambda_{0}\left(n_{1}\right) \lambda_{0}\left(n_{2}\right)\right| \cdot\left|\mathcal{G}\left(1 ; n_{1} n_{2}\right)\right| \cdot\left|\frac{\lambda_{g}\left(\frac{n_{1} n_{2}}{\left(n_{1}, n_{2}\right)^{2}}\right)}{\left(\frac{n_{1} n_{2}}{\left(n_{1}, n_{2}\right)^{2}}\right)^{1 / 2}}\right|\left(\frac{n_{1} n_{2}}{M}\right)^{\alpha}
\end{aligned}
$$

for any $\alpha>0$, which is chosen optimally later.

Write $n=n_{1} n_{2}$ to express the double sum as a single sum over $n$, and note that by the fact that $r(\cdot)$ is supported only on square-free integers coprime to $N$, the only integers $n$ which contribute to the sum over $n$ satisfy $p^{3} \nmid n$ and $(n, N)=1$. Hence, by the multiplicativity of $r(\cdot)$ we can express the sum over $n$ as an Euler product, and the expression on the right-hand side of the previous display equals

$$
\begin{aligned}
& =X M^{-\alpha} \prod_{L^{2} \leq p \leq L^{4}}\left(1+2 r(p) p^{\alpha-1 / 2}|\mathcal{G}(1 ; p)| \cdot \frac{\left|\lambda_{0}(p) \lambda_{g}(p)\right|}{\sqrt{p}}+r(p)^{2} p^{2 \alpha}\left|\mathcal{G}\left(1 ; p^{2}\right)\right| \cdot\left|\lambda_{0}(p)\right|^{2}\right) \\
& \ll X M^{-\alpha} \exp \left(\sum_{L^{2} \leq p \leq L^{4}}\left(\frac{8 L p^{\alpha-1}}{\log p}+\frac{4 L^{2} p^{2 \alpha-1}}{(\log p)^{2}}\right)\left(1+O\left(\frac{1}{p}\right)\right)\right),
\end{aligned}
$$

where the last estimate follows from Deligne's bound $\left|\lambda_{g}(p)\right| \leq 2$, the fact that $\mathcal{G}\left(1 ; p^{k}\right)=$ $1+O(1 / p)$, and the definition of $r(n)$.

Let us now choose $\alpha=1 /(8 \log L)$. Then by the prime number theorem and partial summation, the previous is

$$
\ll X \cdot \exp \left(-\frac{\log M}{8 \log L}+\frac{8 L}{\log L}+\frac{4 L^{2}}{(\log L)^{2}}\right) \ll X
$$

by the choices $L=\frac{1}{8} \sqrt{\log M \log \log M}$ and $M=X^{1 / 24}$. From the foregoing arguments we deduce that

$$
\begin{aligned}
& \sum_{d \in \mathcal{D}_{N, \eta}} L\left(\frac{1}{2}, g \otimes \chi_{d}\right)|R(d)|^{2} \Phi\left(\frac{|d|}{X}\right) \\
& =A_{g, \eta}(\Phi) \cdot X \cdot \prod_{L^{2} \leq p \leq L^{4}}\left(1+2 r(p) \mathcal{G}(1 ; p) \cdot \frac{\lambda_{0}(p) \lambda_{g}(p)}{\sqrt{p}}+r(p)^{2} \mathcal{G}\left(1 ; p^{2}\right) \cdot \lambda_{0}(p)^{2}\right) \\
& \quad+O_{k, N, \Phi}(X) \\
& =A_{g, \eta}(\Phi) \cdot X \cdot \mathcal{R} \cdot \exp \left(2 \sum_{L^{2} \leq p \leq L^{4}} \frac{r(p) \lambda_{0}(p) \lambda_{g}(p)}{\sqrt{p}}+O_{k, N, \Phi}\left(\frac{L}{(\log L)^{3}}\right)\right)+O_{k, N, \Phi}(X),
\end{aligned}
$$

where the last step follows exactly as in $[21, \S 6]$.

We now apply this result with $g=g_{f}$, where $f \in \mathcal{B}_{k+\frac{1}{2}, 4 \ell}^{\text {new }}$ with $\ell \mid N$. For the estimate (31) we choose $\Phi$ to be supported on the interval [1,2] such that $\Phi(t)=1$ for $t \in[11 / 10,19 / 10]$ 
and satisfying $0 \leq \Phi(t) \leq 1$. For the estimate (32) we choose $\Phi$ to be supported in $[1 / 2,5 / 2]$ with $\Phi(t)=1$ for $t \in[1,2]$ and again satisfying $0 \leq \Phi(t) \leq 1$. These choices lead to

$$
\begin{aligned}
& \sum_{d \in \mathcal{D}_{N, \eta}(X)} L\left(\frac{1}{2}, g_{0} \otimes \chi_{d}\right)|R(d)|^{2} \\
& \geq \frac{4 X \mathcal{R}}{5 N} \cdot L_{g_{0}, \eta}\left(\frac{1}{2}\right) L\left(1, \operatorname{Sym}^{2} g_{0}\right) \cdot \exp \left(2 \sum_{L^{2} \leq p \leq L^{4}} \frac{r(p) \lambda_{0}(p)^{2}}{\sqrt{p}}+O\left(\frac{L}{(\log L)^{3}}\right)\right) \\
& \quad+O_{k, N}(X)
\end{aligned}
$$

for $f=f_{0}$ and

$$
\begin{aligned}
& \sum_{d \in \mathcal{D}_{N, \eta}(X)} L\left(\frac{1}{2}, g_{f} \otimes \chi_{d}\right)|R(d)|^{2} \\
& \leq \frac{X \mathcal{R}}{N} \cdot L_{g_{f}, \eta}\left(\frac{1}{2}\right) L\left(1, \operatorname{Sym}^{2} g_{f}\right) \exp \left(2 \sum_{L^{2} \leq p \leq L^{4}} \frac{r(p) \lambda_{0}(p) \lambda_{g_{f}}(p)}{\sqrt{p}}+O\left(\frac{L}{(\log L)^{3}}\right)\right) \\
& \quad+O_{k, N}(X)
\end{aligned}
$$

for $f \neq f_{0}$.

To obtain the estimates (31) and (32) we use partial summation and the following estimate, which is a consequence of the Rankin-Selberg theory [69, Theorem 3]:

Lemma 4.4. With the notation as given, we have

$$
\sum_{p \leq x} \lambda_{0}(p) \lambda_{g_{f}}(p) \log p=x \cdot 1_{f=f_{0}}+o(x) .
$$

\section{Siegel cusp forms of degree 2}

In this section we first review various properties of Siegel cusp forms of degree 2 and then go on to prove our main results stated in the introduction.

\subsection{Preliminaries}

Denote by $J$ the $4 \times 4$ matrix given by $J=\left(\begin{array}{cc}0 & I_{2} \\ -I_{2} & 0\end{array}\right)$, where $I_{2}$ is the identity matrix of size 2. Define the algebraic groups $\mathrm{GSp}_{4}$ and $\mathrm{Sp}_{4}$ over $\mathbb{Z}$ by

$$
\begin{gathered}
\operatorname{GSp}_{4}(R)=\left\{g \in \mathrm{GL}_{4}(R) \mid{ }^{t} g J g=\mu_{2}(g) J, \mu_{2}(g) \in R^{\times}\right\} \\
\operatorname{Sp}_{4}(R)=\left\{g \in \operatorname{GSp}_{4}(R) \mid \mu_{2}(g)=1\right\}
\end{gathered}
$$

for any commutative ring $R$. The Siegel upper half-space $\mathbb{H}_{2}$ of degree 2 is defined by

$$
\mathbb{H}_{2}=\left\{Z \in \operatorname{Mat}_{2 \times 2}(\mathbb{C}) \mid Z={ }^{t} Z, \operatorname{Im}(Z) \text { is positive definite }\right\} \text {. }
$$


Let $k$ and $N$ be positive integers. Let $\Gamma_{0}^{(2)}(N) \subseteq \operatorname{Sp}_{4}(\mathbb{Z})$ denote the Siegel congruence subgroup of level $N$ - that is,

$$
\Gamma_{0}^{(2)}(N)=\operatorname{Sp}_{4}(\mathbb{Z}) \cap\left(\begin{array}{ccc}
\mathbb{Z} & \mathbb{Z} & \mathbb{Z} \\
\mathbb{Z} & \mathbb{Z} \\
N \mathbb{Z} & N \mathbb{Z} \\
N \mathbb{Z} & N \mathbb{Z} & \mathbb{Z}
\end{array}\right)
$$

We define

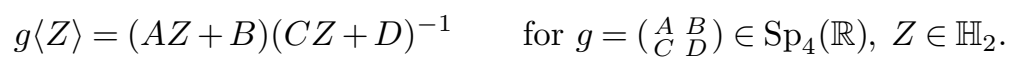

We let $J(g, Z)=C Z+D$. Let $S_{k}\left(\Gamma_{0}^{(2)}(N)\right)$ denote the space of Siegel cusp forms of weight $k$ and level $N$; precisely, they consist of the holomorphic functions $F$ on $\mathbb{H}_{2}$ which satisfy the relation

$$
F(\gamma\langle Z\rangle)=\operatorname{det}(J(\gamma, Z))^{k} F(Z)
$$

for $\gamma \in \Gamma_{0}^{(2)}(N), Z \in \mathbb{H}_{2}$, and vanish at all the cusps. Any $F$ in $S_{k}\left(\Gamma_{0}^{(2)}(N)\right)$ has a Fourier expansion

$$
F(Z)=\sum_{S \in \Lambda_{2}} a(F, S) e^{2 \pi i \operatorname{Tr}(S Z)}
$$

with $\Lambda_{2}$ defined in equation (2). We have the relation

$$
a(F, T)=\operatorname{det}(A)^{k} a\left(F,{ }^{t} A T A\right)
$$

for $A \in \mathrm{GL}_{2}(\mathbb{Z})$. In particular, the Fourier coefficient $a(F, T)$ depends only on the $\mathrm{SL}_{2}(\mathbb{Z})$ equivalence class of $T$. We say that a matrix $S \in \Lambda_{2}$ is fundamental if $\operatorname{disc}(S)=-4 \operatorname{det}(S)$ is a fundamental discriminant. Given a fundamental discriminant $d<0$ and $K=\mathbb{Q}(\sqrt{d})$, let $\mathrm{Cl}_{K}$ denote the ideal class group of $K$. It is well known that the $\mathrm{SL}_{2}(\mathbb{Z})$-equivalence classes of matrices in $\Lambda_{2}$ of discriminant $d$ can be identified with $\mathrm{Cl}_{K}$; so the expression $\sum_{S \in \mathrm{Cl}_{K}} a(F, S) \Lambda(S)$ makes sense for each $\Lambda \in \widehat{\mathrm{Cl}_{K}}$.

\subsection{Constructing half-integral-weight forms}

Each $F$ in $S_{k}\left(\Gamma_{0}^{(2)}(N)\right)$ has a Fourier-Jacobi expansion $F(Z)=\sum_{m>0} \phi_{m}(\tau, z) e^{2 \pi i m \tau^{\prime}}$, where we write $Z=\left(\begin{array}{cc}\tau & z \\ z & \tau^{\prime}\end{array}\right)$ and for each $m>0$,

$$
\phi_{m}(\tau, z)=\sum_{\substack{n, r \in \mathbb{Z} \\
4 n m>r^{2}}} a\left(F,\left(\begin{array}{cc}
n & r / 2 \\
r / 2 & m
\end{array}\right)\right) e^{2 \pi i(n \tau+r z)} \in J_{k, m}^{\text {cusp }}(N) .
$$

Here $J_{k, m}^{\text {cusp }}(N)$ denotes the space of Jacobi cusp forms of weight $k$, level $N$, and index $m$.

Given a primitive matrix $S=\left(\begin{array}{cc}a & b / 2 \\ b / 2 & c\end{array}\right) \in \Lambda_{2}($ i.e., $\operatorname{gcd}(a, b, c)=1)$, we let $\mathcal{P}(S)$ denote the set of primes of the form $a x^{2}+b x y+c y^{2}$. The set $\mathcal{P}(S)$ is infinite; indeed, by [28, Theorem 1(i)],

$$
|\{p \in \mathcal{P}(S): p \leq X\}| \gg_{S} \frac{X}{\log X} .
$$


For each prime $p$ dividing $N$, define the operator $U(p)$ acting on the space $S_{k}\left(\Gamma_{0}^{(2)}(N)\right)$ via

$$
a(U(p) F, S)=a(F, p S)
$$

Lemma 5.1. Let $k>2$ be even and $N$ be square-free. Let $F \in S_{k}\left(\Gamma_{0}^{(2)}(N)\right)$ be an eigenfunction of the $U(p)$ operator for each prime $p \mid N$ (note that if $N=1$, this condition is trivially true).

i) Then there exists $S_{0}=\left(\begin{array}{cc}a & b / 2 \\ b / 2 & c\end{array}\right) \in \Lambda_{2}$ such that $a\left(F, S_{0}\right) \neq 0$ and $d_{0}=b^{2}-4 a c$ is odd and square-free (and hence $S_{0}$ is fundamental).

ii) Let $S_{0}, d_{0}$ be as before and let $p \nmid 2 N d_{0}$ be a prime such that $p \in \mathcal{P}\left(S_{0}\right)$. Put

$$
h_{p}(z)=\sum_{m=1}^{\infty} a(m) e^{2 \pi i m z}, \quad \text { where } a(m)=\sum_{\substack{0 \leq \mu \leq 2 p-1 \\
\mu^{2} \equiv-m(\bmod 4 p)}} a\left(F,\left(\begin{array}{cc}
\frac{m+\mu^{2}}{4 p} & \frac{\mu}{2} \\
\frac{\mu}{2} & p
\end{array}\right)\right) .
$$

Then $0 \neq h_{p} \in S_{k-\frac{1}{2}}^{+}(4 p N)$. Furthermore, for each $m>0$ such that $\operatorname{gcd}(m, 4 p)=1$,

$$
a(m)=2 a\left(F,\left(\begin{array}{cc}
\frac{m+\mu_{0}^{2}}{4 p} & \frac{\mu_{0}}{2} \\
\frac{\mu_{0}}{2} & p
\end{array}\right)\right)
$$

where $\mu_{0}$ is any integer satisfying $\mu_{0}^{2} \equiv-m(\bmod 4 p)$; if no such $\mu_{0}$ exists, then $a(m)=0$.

Proof. The claim that there exists $S_{0}=\left(\begin{array}{cc}a & b / 2 \\ b / 2 & c\end{array}\right) \in \Lambda_{2}$ such that $a\left(F, S_{0}\right) \neq 0$ and $d_{0}=b^{2}-4 a c$ is odd and square-free follows from [58, Theorem 2.2].

Now let $p \in \mathcal{P}\left(S_{0}\right), p \nmid 2 N d_{0}$. The fact that $h_{p} \in S_{k-\frac{1}{2}}(4 p N)$ follows from $\phi_{p} \in J_{k, p}^{\text {cusp }}(N)$ and [41, Theorem 4.8]; by definition, $h_{p}$ belongs to the Kohnen plus space. Next we prove equation (51). Let $\operatorname{gcd}(m, 4 p)=1$. Observe that the $\operatorname{sum} \sum_{\substack{0 \leq \mu \leq 2 p-1 \\ \mu^{2} \equiv-m(\bmod 4 p)}}$ is nonempty if and only if $-m$ is a square modulo $4 p$, in which case the sum has exactly two terms. Indeed, we get

$$
a(m)=a\left(F,\left(\begin{array}{cc}
\frac{m+\mu_{0}^{2}}{4 p} & \frac{\mu_{0}}{2} \\
\frac{\mu_{0}}{2} & p
\end{array}\right)\right)+a\left(F,\left(\begin{array}{cc}
\frac{m+\mu_{1}^{2}}{4 p} & \frac{\mu_{1}}{2} \\
\frac{\mu_{1}}{2} & p
\end{array}\right)\right)
$$

where $0 \leq \mu_{0} \leq 2 p-1$ satisfies $\mu_{0}^{2} \equiv-m(\bmod 4 p)$ and $\mu_{1}=2 p-\mu_{0}$. Using equations (47) and (52) and the identity $\left(\begin{array}{cc}1 & -1 \\ 0 & -1\end{array}\right)\left(\begin{array}{cc}\frac{m+\mu_{0}^{2}}{4 p} & \frac{\mu_{0}}{2} \\ \frac{\mu_{0}}{2} & p\end{array}\right)\left(\begin{array}{cc}1 & 0 \\ -1 & -1\end{array}\right)=\left(\begin{array}{cc}\frac{m+\mu_{1}^{2}}{4 p} & \frac{\mu_{1}}{2} \\ \frac{\mu_{1}}{2} & p\end{array}\right)$, we obtain equation (51).

It remains to show $h_{p} \neq 0$, for which we will show that $a\left(-d_{0}\right) \neq 0$. Let $x_{0}, y_{0}$ be integers such that $c x_{0}^{2}+b x_{0} y_{0}+a y_{0}^{2}=p$. Since $\operatorname{gcd}\left(x_{0}, y_{0}\right)=1$, we may pick integers $x_{1}, y_{1}$ such 
that $A=\left(\begin{array}{ll}y_{1} & y_{0} \\ x_{1} & x_{0}\end{array}\right) \in \mathrm{SL}_{2}(\mathbb{Z})$. Then $S^{\prime}={ }^{t} A S A$ is $\mathrm{SL}_{2}(\mathbb{Z})$-equivalent to $S_{0}$ and has the form $S^{\prime}=\left(\begin{array}{cc}\frac{-d_{0}+\mu_{0}^{2}}{4 p} & \frac{\mu_{0}}{2} \\ \frac{\mu_{0}}{2} & p\end{array}\right) \in \Lambda_{2}$. By equation $(47), a\left(F, S^{\prime}\right) \neq 0$ since $a\left(F, S_{0}\right) \neq 0$. Hence by equation $(51), a\left(-d_{0}\right)=2 a\left(F, S^{\prime}\right) \neq 0$.

\subsection{Proofs of Theorems A and B}

We are now ready to prove Theorems A and B in slightly stronger forms.

Theorem 5.2. Let $F \in S_{k}\left(\Gamma_{0}^{(2)}(N)\right)$ with $k>2$ even and $N$ odd and square-free. Assume that $F$ is an eigenfunction of the $U(p)$ operator for each prime $p \mid N$, and that $F$ has real Fourier coefficients. Then there exists a set $\mathcal{P}$ of primes satisfying $|\{p \in \mathcal{P}: p \leq X\}| \gg_{F}$ $\frac{X}{\log X}$ such that given $\varepsilon>0$ and $p \in \mathcal{P}$, there exist $M \geq 2$ (depending only on $F$ and $p$ ) and $X_{0} \geq 1$ (depending on $F, p$, and $\varepsilon$ ) such that for all $X \geq X_{0}$, there are $r_{X} \geq X^{1-\varepsilon}$ matrices $S_{1}, S_{2}, \ldots, S_{r_{X}} \in \Lambda_{2}$ satisfying the following:

i) $X \leq\left|\operatorname{disc}\left(S_{1}\right)\right|<\left|\operatorname{disc}\left(S_{2}\right)\right|<\cdots<\left|\operatorname{disc}\left(S_{r_{X}}\right)\right| \leq M X$.

ii) For each $1 \leq i \leq r_{X}, S_{i}=\left(\begin{array}{cc}* & * \\ * & p\end{array}\right)$, and $\operatorname{disc}\left(S_{i}\right)$ is a square-free integer coprime to $2 N$.

iii) For each $1 \leq i \leq r_{X}-1, a\left(F, S_{i}\right) a\left(F, S_{i+1}\right)<0$.

Proof. Using Lemma 5.1, we fix a fundamental matrix $S_{0}$ such that $a\left(F, S_{0}\right) \neq 0$. Take $\mathcal{P}=\mathcal{P}\left(S_{0}\right)$; then the estimate $|\{p \in \mathcal{P}: p \leq X\}| \gg \frac{X}{\log X}$ follows from the bound (49). Given any $p \in \mathcal{P}$, let $f=h_{p}$ be as in Lemma 5.1 , so that $0 \neq f \in S_{k-\frac{1}{2}}^{+}(4 p N)$ and the coefficients of $f$ are all real, since the coefficients of $F$ are.

By Theorem 3.1, there exists $M \geq 2$ such that for any $\varepsilon>0$, the sequence $\left\{a(f, n) \mu^{2}(n)\right\}_{\substack{X \leq n \leq M X \\(n, 2 N)=1}}$ has $\geq C_{f, M, \varepsilon} X^{1-\varepsilon / 2}$ sign changes for some constant $C_{f, M, \varepsilon}$. Pick $X_{0} \geq 1$ such that for all $X \geq X_{0}$ we have $X^{\varepsilon / 2} \geq \frac{1}{C_{f, M, \varepsilon}}$. Then for all $X \geq X_{0}$, there exists $r_{X} \geq X^{1-\varepsilon}$ and an increasing sequence $\left(n_{i}\right)_{1 \leq i \leq r_{X}}$ of odd square-free integers satisfying $a\left(f, n_{i}\right) a\left(f, n_{i+1}\right)<0$. For each $n_{i}$ as discussed, equation (51) tells us that $a\left(f, n_{i}\right)=2 a\left(F, S_{i}\right)$ for some $S_{i}=\left(\begin{array}{cc}* & * \\ * & p\end{array}\right) \in \Lambda_{2}$ with $\left|\operatorname{disc}\left(S_{i}\right)\right|=n_{i}$. This completes the proof.

Theorem 5.3. Let $F \in S_{k}\left(\Gamma_{0}^{(2)}(N)\right)$ with $k>2$ even and $N$ odd and square-free. Assume that $F$ is an eigenfunction of the $U(p)$ operator for each prime $p \mid N$. Then there exists a set $\mathcal{P}$ of primes satisfying $|\{p \in \mathcal{P}: p \leq X\}| \gg_{F} \frac{X}{\log X}$ such that given $\varepsilon>0$ and $p \in \mathcal{P}$, one can find $X_{0} \geq 1$ (depending on $F, p$, and $\varepsilon$ ) such that for all $X \geq X_{0}$, there are $r_{X} \geq X^{1-\varepsilon}$ matrices $S_{1}, S_{2}, \ldots, S_{r_{X}} \in \Lambda_{2}$ satisfying the following:

i) For each $1 \leq i \leq r_{X}, S_{i}=\left(\begin{array}{cc}* & * \\ * & p\end{array}\right)$, and $\operatorname{disc}\left(S_{i}\right)$ is a square-free integer coprime to $2 N$. 
ii) $X \leq\left|\operatorname{disc}\left(S_{1}\right)\right|<\left|\operatorname{disc}\left(S_{2}\right)\right|<\cdots<\left|\operatorname{disc}\left(S_{r_{X}}\right)\right| \leq 2 X$
iii) For each $1 \leq i \leq r_{X},\left|a\left(F, S_{i}\right)\right| \geq\left|\operatorname{disc}\left(S_{i}\right)\right|^{\frac{k}{2}-\frac{3}{4}} \exp \left(\frac{1}{82} \sqrt{\frac{\log \left|\operatorname{disc}\left(S_{i}\right)\right|}{\log \log \left|\operatorname{disc}\left(S_{i}\right)\right|}}\right)$

Proof. The proof is essentially identical to the proof of Theorem 5.2, except that we use Theorem 4.1 (rather than Theorem 3.1) on $f=h_{p}$.

\section{4. $L$-functions of Siegel cusp forms}

For the rest of this section, we assume that $k>2$.

Given an irreducible cuspidal automorphic representation $\pi$ of $\operatorname{GSp}_{4}(\mathbb{A})$, we let $L(s, \pi)$ denote the associated degree $4 L$-function (known as the 'spin' $L$-function). Furthermore, we let $L(s, \operatorname{std}(\pi))$ denote the associated degree $5 L$-function (the 'standard' $L$-function) and $L(s, \operatorname{ad}(\pi))$ denote the associated degree $10 L$-function (the 'adjoint' $L$-function). Each of these $L$-functions is defined as an Euler product with the local $L$-factor at each prime (including the ramified primes) constructed via the associated representation of the dual group $\mathrm{GSp}_{4}(\mathbb{C})$ using the local Langlands correspondence (which is known for $\mathrm{GSp}_{4}$ by the work of Gan and Takeda [19]). More precisely, for $n=4,5,10$, let $\rho_{n}$ denote the irreducible $n$-dimensional representation of $\operatorname{GSp}_{4}(\mathbb{C})$ given as follows: $\rho_{4}$ is the inclusion $\mathrm{GSp}_{4}(\mathbb{C}) \hookrightarrow \mathrm{GL}_{4}(\mathbb{C}), \rho_{5}$ is the map defined in $[52, \mathrm{~A} .7]$, and $\rho_{10}$ is the adjoint representation of $\mathrm{GSp}_{4}(\mathbb{C})$ on the Lie algebra of $\mathrm{Sp}_{4}(\mathbb{C})$. Then $L(s, \pi), L(s, \operatorname{std}(\pi))$, and $L(s, \operatorname{ad}(\pi))$ correspond to the representations $\rho_{4}, \rho_{5}$, and $\rho_{10}$, respectively.

We say that an element $F$ of $S_{k}\left(\Gamma_{0}^{(2)}(N)\right)$ gives rise to an irreducible representation if its adelization (in the sense of $[57, \S 3]$ ) generates an irreducible cuspidal automorphic representation of $\mathrm{GSp}_{4}(\mathbb{A})$. The automorphic representation associated to any such $F$ is of trivial central character and hence may be viewed as an automorphic representation of $\mathrm{PGSp}_{4}(\mathbb{A}) \simeq \mathrm{SO}_{5}(\mathbb{A})$. It can be checked [57, Proposition 3.11] that if $F$ gives rise to an irreducible representation, then $F$ is an eigenform of the local Hecke algebras at all primes not dividing $N$. In addition, we say that such an $F$ is factorizable if its adelization corresponds to a factorizable vector in the representation generated.

We say that an irreducible cuspidal automorphic representation $\pi$ of $\mathrm{GSp}_{4}(\mathbb{A})$ arises from $S_{k}\left(\Gamma_{0}^{(2)}(N)\right)$ if there exists $F \in S_{k}\left(\Gamma_{0}^{(2)}(N)\right.$ ) whose adelization generates $V_{\pi}$ (in which case, by definition, $F$ gives rise to the irreducible representation $\pi$, which therefore must be of trivial central character by the previous comments). We say that an irreducible cuspidal automorphic representation $\pi$ of $\operatorname{GSp}_{4}(\mathbb{A})$ is 'CAP' if it is nearly equivalent to a constituent of a global induced representation of a proper parabolic subgroup of $\mathrm{GSp}_{4}(\mathbb{A})$. If a CAP $\pi$ arises from $S_{k}\left(\Gamma_{0}^{(2)}(N)\right)$, then by [47, Corollary 4.5] it is associated to the Siegel parabolic (i.e., it is of Saito-Kurokawa type). Such a $\pi$ is nontempered at almost all primes (in particular, it violates the Ramanujan conjecture). On the other hand, if $\pi$ arises from $S_{k}\left(\Gamma_{0}^{(2)}(N)\right)$ and is not CAP, then $\pi$ satisfies the Ramanujan conjecture by a famous result of Weissauer [68, Theorem 3.3].

Thus, the space $S_{k}\left(\Gamma_{0}^{(2)}(N)\right)$ has a natural decomposition into orthogonal subspaces

$$
S_{k}\left(\Gamma_{0}^{(2)}(N)\right)=S_{k}\left(\Gamma_{0}^{(2)}(N)\right)^{\mathrm{CAP}} \oplus S_{k}\left(\Gamma_{0}^{(2)}(N)\right)^{\mathrm{T}}
$$


where $S_{k}\left(\Gamma_{0}^{(2)}(N)\right)^{\mathrm{CAP}}$ is spanned by forms $F$ which give rise to irreducible representations of Saito-Kurokawa type, and $S_{k}\left(\Gamma_{0}^{(2)}(N)\right)^{\mathrm{T}}$ is its orthogonal complement, spanned by forms $F$ which give rise to irreducible representations that are not of Saito-Kurokawa type. Furthermore, one can get a basis of each of the spaces $S_{k}\left(\Gamma_{0}^{(2)}(N)\right)^{\mathrm{CAP}}$ and $S_{k}\left(\Gamma_{0}^{(2)}(N)\right)^{\mathrm{T}}$ in terms of factorizable forms. We refer the reader to [15, $\S \S 3.1$ and 3.2$]$ for further comments related to the foregoing discussion.

If $\pi$ arises from $S_{k}\left(\Gamma_{0}^{(2)}(N)\right)$ and is of Saito-Kurokawa type, then there exist a representation $\pi_{0}$ of $\mathrm{GL}_{2}(\mathbb{A})$ and a Dirichlet character $\chi_{0}$ satisfying $\chi_{0}^{2}=1$ such that $L^{N}(s, \pi)=L^{N}\left(s, \pi_{0}\right) L^{N}\left(s+1 / 2, \chi_{0}\right) L^{N}\left(s-1 / 2, \chi_{0}\right)$. Additionally, if $N$ is square-free, then only $\chi_{0}=1$ is possible, by a well-known result of Borel [8], and so in this case we have $L^{N}(s, \pi)=L^{N}\left(s, \pi_{0}\right) \zeta^{N}(s+1 / 2) \zeta^{N}(s-1 / 2)$. There exists another typical situation where the $L$-function factors: we say that a $\pi$ arising from $S_{k}\left(\Gamma_{0}^{(2)}(N)\right)$ is of Yoshida type if there are representations $\pi_{1}$ and $\pi_{2}$ of $\mathrm{GL}_{2}(\mathbb{A})$ such that $L(s, \pi)=L\left(s, \pi_{1}\right) L\left(s, \pi_{2}\right)$; in this case (after possibly swapping $\pi_{1}$ and $\pi_{2}$ ), $\pi_{1}$ arises from a classical holomorphic newform of weight 2 and $\pi_{2}$ arises from a classical holomorphic newform of weight $2 k-2$ (see $[57, \S 4]$ for more details). We let $S_{k}\left(\Gamma_{0}^{(2)}(N)\right)^{\mathrm{Y}}$ denote the subspace of $S_{k}\left(\Gamma_{0}^{(2)}(N)\right)^{\mathrm{T}}$ spanned by forms which give rise to an irreducible representation of Yoshida type, and let $S_{k}\left(\Gamma_{0}^{(2)}(N)\right)^{\mathrm{G}}$ - which represents the general type - denote the orthogonal complement of $S_{k}\left(\Gamma_{0}^{(2)}(N)\right)^{\mathrm{Y}}$ in $S_{k}\left(\Gamma_{0}^{(2)}(N)\right)^{\mathrm{T}}$. So we get the following key orthogonal decomposition into subspaces:

$$
S_{k}\left(\Gamma_{0}^{(2)}(N)\right)=S_{k}\left(\Gamma_{0}^{(2)}(N)\right)^{\mathrm{CAP}} \oplus S_{k}\left(\Gamma_{0}^{(2)}(N)\right)^{\mathrm{Y}} \oplus S_{k}\left(\Gamma_{0}^{(2)}(N)\right)^{\mathrm{G}} .
$$

In the notation of [59], the three subspaces on the right-hand side correspond to the global Arthur packets of type $(\mathbf{P}),(\mathbf{Y})$, and $(\mathbf{G})$, respectively. In the sequel we will be mostly concerned with the space $S_{k}\left(\Gamma_{0}^{(2)}(N)\right)^{\mathrm{G}}$, because the other two spaces are easier to handle. The following proposition collects together some relevant facts about the associated $L$ functions that follow from the work of Arthur [2]:

Proposition 5.4. Suppose that $F \in S_{k}\left(\Gamma_{0}^{(2)}(N)\right)^{\mathrm{G}}$ gives rise to an irreducible representation $\pi$.

i) The representation $\pi$ has a strong functorial lifting to an irreducible cuspidal automorphic representation $\Pi_{4}$ of $\mathrm{GL}_{4}(\mathbb{A})$. In particular, if $\sigma$ is any irreducible automorphic representation of $\mathrm{GL}_{n}(\mathbb{A})$, then we have an equality of degree $4 n$ Rankin-Selberg L-functions $L(s, \pi \times \sigma)=L\left(s, \Pi_{4} \times \sigma\right)$, and therefore $L(s, \pi \times \sigma)$ satisfies the usual properties ${ }^{6}$ of an $L$-function. If $n \leq 3$, then $L(s, \pi \times \sigma)$ is entire.

ii) The representation $\pi$ has a strong functorial lifting to an irreducible automorphic representation $\Pi_{5}$ of $\mathrm{GL}_{5}(\mathbb{A})$. In particular, if $\sigma$ is any irreducible automorphic representation of $\mathrm{GL}_{n}(\mathbb{A})$, then we have an equality of degree $5 n$ Rankin-Selberg

${ }^{6} \mathrm{By}$ this we mean that this $L$-function has meromorphic continuation to the entire complex plane, satisfies the standard functional equation taking $s \mapsto 1-s$, has an Euler product, and is bounded in vertical strips (in particular, the $L$-function is in the extended Selberg class). 
$L$-functions $L(s, \operatorname{std}(\pi) \times \sigma)=L\left(s, \Pi_{5} \times \sigma\right)$, and therefore $L(s, \operatorname{std}(\pi) \times \sigma)$ satisfies the usual properties of an $L$-function. If $n \leq 2$, and $\sigma$ has the property that the set of finite primes where it is ramified either is empty or contains at least one prime not dividing $N$, then $L(s, \operatorname{std}(\pi) \times \sigma)$ is entire.

iii) The degree $10 \mathrm{~L}$-function $L(s, \operatorname{ad}(\pi))$ satisfies the usual properties of an L-function, is entire, and has no zeros on the line $\operatorname{Re}(s)=1$.

Proof. Since $F$ corresponds to the 'general' Arthur parameter, Arthur's work [2] (see also $[59, \S 1.1])$ shows that $\pi$ has a strong lifting to a cuspidal automorphic representation $\Pi_{4}$ of $\mathrm{GL}_{4}$; clearly $\Pi_{4}$ has trivial central character, since $\pi$ does. It is known from [2, Theorem 1.5.3] that $\Pi_{4}$ is self-dual and symplectic. ${ }^{7}$ The fact that the lifting is 'strong' - that is, corresponds to a local lift at all places - implicitly uses the fact that the local Arthur parameters coincide with the local Langlands parameters of Gan and Takeda [19]; this consistency of local parameters follows from [10]. The required properties of $L(s, \pi \times \sigma)$ now follow from the Rankin-Selberg theory of $\mathrm{GL}_{n}$.

For the second assertion, let $\wedge^{2}$ denote the exterior square, and note that $\wedge^{2} \rho_{4}=\mathbf{1}+\rho_{5}$; hence

$$
L\left(s, \Pi_{4}, \wedge^{2}\right)=L(s, \operatorname{std}(\pi)) \zeta(s) .
$$

Recall that $L\left(s, \Pi_{4} \times \Pi_{4}\right)=L\left(s, \Pi_{4}, \mathrm{Sym}^{2}\right) L\left(s, \Pi_{4}, \wedge^{2}\right)$. Since $\Pi_{4}$ is symplectic, $L\left(s, \Pi_{4}, \wedge^{2}\right)$ has a simple pole at $s=1$ and $L\left(s, \Pi_{4}, \mathrm{Sym}^{2}\right)$ is an entire function by [9, Theorem 7.5]. It follows from equation (54) that $L(s, \operatorname{std}(\pi))$ is holomorphic and nonzero at $s=1$. Together with [20, Theorem 2], we obtain that $L(s, \operatorname{std}(\pi))$ has no poles on $\operatorname{Re}(s)=1$. On the other hand, by [32, Theorem A] and [26], $L\left(s, \Pi_{4}, \wedge^{2}\right)$ is the $L$ function of an automorphic representation of $\mathrm{GL}_{6}(\mathbb{A})$ of the form $\operatorname{Ind}\left(\tau_{1} \otimes \cdots \otimes \tau_{m}\right)$, where $\tau_{1}, \ldots, \tau_{m}$ are unitary, cuspidal, automorphic representations of $\mathrm{GL}_{n_{i}}(\mathbb{A}), n_{1}+\cdots+n_{m}=6$. Since $L\left(s, \Pi_{4}, \wedge^{2}\right)$ has a simple pole at $s=1$, it follows that exactly one of the $\tau_{i}-$ say $\tau_{m}$ - is the trivial representation of $\mathrm{GL}_{1}(\mathbb{A})$. Canceling out one $\zeta$ factor, we see that

$$
L(s, \operatorname{std}(\pi))=L\left(s, \tau_{1}\right) \cdots L\left(s, \tau_{m-1}\right) .
$$

So we take $\Pi_{5}=\operatorname{Ind}\left(\tau_{1} \otimes \cdots \otimes \tau_{m-1}\right)$; the foregoing discussion shows that $\Pi_{5}$ is a (strong) lifting of $\pi$ from $\mathrm{GSp}_{4}(\mathbb{A})$ to $\mathrm{GL}_{5}(\mathbb{A})$ with respect to the map $\rho_{5}$ of dual groups $\mathrm{GSp}_{4}(\mathbb{C}) \rightarrow$ $\mathrm{SO}_{5}(\mathbb{C}) \subset \mathrm{GL}_{5}(\mathbb{C})$. Observe that each $\tau_{i}$ is unitary, cuspidal, and unramified outside primes dividing $N$. By Rankin-Selberg theory, $L\left(s, \Pi_{5} \times \sigma\right)=\prod_{i=1}^{m-1} L\left(s, \tau_{i} \times \sigma\right)$ satisfies the usual properties of an $L$-function. To complete the proof, it suffices to show that if $\sigma$ is an irreducible cuspidal representation of $\mathrm{GL}_{1}(\mathbb{A})$ or $\mathrm{GL}_{2}(\mathbb{A})$ such that the set of ramification primes for $\sigma$ either is empty or contains at least one prime outside $N$, then $\tau_{i} \not \hat{\sigma}$ for each $i$. First, consider the case where $\sigma$ is a character, in which case we are reduced to $n_{i}=1$. In this case, since $\tau_{i}$ is unramified outside $N$, the assumption on $\sigma$ means that the situation $\tau_{i} \simeq \hat{\sigma}$ will force $\tau_{i}$ to be unramified everywhere, in which case the right-hand side of equation (55) would have a pole on $\operatorname{Re}(s)=1$. This contradicts

${ }^{7}$ Recall that a self-dual representation $\Pi$ of $\operatorname{GL}_{n}(\mathbb{A})$ is said to be symplectic if $L\left(s, \operatorname{Sym}^{2} \Pi\right)$ is holomorphic at $s=1$. 
the observation from before that $L(s, \operatorname{std}(\pi))$ has no poles on $\operatorname{Re}(s)=1$. Next consider the case where $\sigma$ is a cuspidal representation of $\mathrm{GL}_{2}(\mathbb{A})$. An identical argument to the foregoing reduces us to the case where $\tau_{i} \simeq \hat{\sigma}$ is unramified at all finite primes. The easy relation $\wedge^{2} \rho_{5}=\operatorname{Sym}^{2} \rho_{4}=\rho_{10}$ implies that

$$
L\left(s, \Pi_{5}, \wedge^{2}\right)=L(s, \operatorname{ad}(\pi))=L\left(s, \Pi_{4}, \mathrm{Sym}^{2}\right),
$$

which we know is an entire function. On the other hand, if some $\tau_{i}$ in equation (55) has $n_{i}=2$ and is unramified at all finite primes, then $L\left(s, \Pi_{5}, \wedge^{2}\right)$ will contain a factor of $L\left(s, \omega_{\tau_{i}}\right)$ and so will have a pole on $\operatorname{Re}(s)=1$. This contradiction completes the proof that each $L\left(s, \tau_{i} \times \sigma\right)$ and hence $L\left(s, \Pi_{5} \times \sigma\right)$ is entire.

Finally, the assertion concerning the degree $10 L$-function $L(s, \operatorname{ad}(\pi))$ follows from the identity $(56)$ and the fact that $L\left(s, \Pi_{4}, \mathrm{Sym}^{2}\right)$ represents a holomorphic $L$-function. Since symmetric square $L$-functions are accessible via the Langlands-Shahidi method, the nonvanishing on $\operatorname{Re}(s)=1$ follows (see [60, §5] and [61, Theorem 1.1]).

For our future applications, we will also need the facts that $L(s, \operatorname{ad}(\pi) \times \sigma)$ has the properties of an $L$-function and is entire for certain special automorphic representations $\sigma$ of $\mathrm{GL}_{1}(\mathbb{A})$ or $\mathrm{GL}_{2}(\mathbb{A})$. The next two lemmas achieve this for $\sigma$ a quadratic character or $\sigma$ of the form $\mathcal{A I}\left(\Lambda^{2}\right)$, where $\Lambda$ is a character of $\mathbb{A}_{K}^{\times}, K$ is a quadratic field, and $\mathcal{A I}$ denotes automorphic induction.

Lemma 5.5. Let $K / \mathbb{Q}$ be a quadratic field. Let $F$ and $\pi$ be as in Proposition 5.4 and assume that $N$ is square-free. Then the base change $\pi_{K}$ of $\pi$ to $\mathrm{GSp}_{4}\left(\mathbb{A}_{K}\right)$ is cuspidal. Furthermore, the base change $\Pi_{4, K}$ of $\Pi_{4}$ to $\mathrm{GL}_{4}\left(\mathbb{A}_{K}\right)$ is cuspidal and $\Pi_{4, K}$ is the lifting of $\pi_{K}$ from $\mathrm{GSp}_{4}\left(\mathbb{A}_{K}\right)$ to $\mathrm{GL}_{4}\left(\mathbb{A}_{K}\right)$.

Proof. Let $\pi_{K}$ and $\Pi_{4, K}$ be as in the statement of the lemma. Since $\Pi_{4}$ is the lifting of $\pi$, it follows from the definition of base change that $\Pi_{4, K}$ is the lifting of $\pi_{K}$. Let $\sigma$ be an arbitrary cuspidal automorphic representation of $\mathrm{GL}_{1}\left(\mathbb{A}_{K}\right)$ or $\mathrm{GL}_{2}\left(\mathbb{A}_{K}\right)$. By the definition of lifting, we have

$$
L\left(s, \pi_{K} \times \sigma\right)=L\left(s, \Pi_{4, K} \times \sigma\right),
$$

and so to prove that $\pi_{K}$ and $\Pi_{4, K}$ are cuspidal it suffices to show that $L\left(s, \Pi_{4, K} \times \sigma\right)$ has no poles. By the adjointness formula [48, Proposition 3.1], we have $L\left(s, \Pi_{4, K} \times \sigma\right)=$ $L\left(s, \Pi_{4} \times \mathcal{A I}(\sigma)\right)$. Note that $\mathcal{A I}(\sigma)$ is an automorphic representation of $\mathrm{GL}_{2}(\mathbb{A})$ if $\sigma$ is a character of $\mathbb{A}_{K}^{\times}$, and $\mathcal{A I}(\sigma)$ is an automorphic representation of $\mathrm{GL}_{4}(\mathbb{A})$ if $\sigma$ is a cuspidal representation of $\mathrm{GL}_{2}\left(\mathbb{A}_{K}\right)$. By Proposition 5.4, $L\left(s, \Pi_{4} \times \mathcal{A I}(\sigma)\right)$ is entire when $\sigma$ is a character. This reduces us to the case where $\sigma$ is a cuspidal representation of $\mathrm{GL}_{2}\left(\mathbb{A}_{K}\right)$; in this case, $L\left(s, \Pi_{4, K} \times \sigma\right)=L\left(s, \Pi_{4} \times \mathcal{A I}(\sigma)\right)$ has a pole if and only if $\Pi_{4} \simeq \mathcal{A} \mathcal{I}(\sigma)$ (recall that $\Pi_{4}$ is self-dual). However, by looking at a prime $p$ which ramifies in $K$, we see that this is impossible: at any such prime, the local Langlands parameter of $\Pi_{4, p}$ is the local lifting of a representation of $\mathrm{GSp}_{4}\left(\mathbb{Q}_{p}\right)$ that has a vector fixed by the local Siegel congruence subgroup of level $p$ (since $N$ is square-free), but an inspection of [29, Table 1] tells us that the local parameter of $\mathcal{A} \mathcal{I}\left(\sigma_{p}\right)$ can never equal one of those. Hence we have completed the proof that $L\left(s, \Pi_{4, K} \times \sigma\right)$ has no poles. 
Remark 5.6. This proof crucially relies on the fact that $F$ has square-free level $N$. If $N$ is allowed to be divisible by squares of primes, there indeed exists $F$ whose adelization generates a representation $\pi$ whose base change $\pi_{K}$ (for certain $K$ ) is noncuspidal. Such $F$ are constructed in [29] for $K$ real quadratic and in [4] for $K$ imaginary quadratic.

Lemma 5.7. Let $K / \mathbb{Q}$ be a quadratic field. Let $F$ and $\pi$ be as in Proposition 5.4 and assume that $N$ is square-free. Let $\chi_{K}$ be the quadratic Dirichlet character associated to the extension $K / \mathbb{Q}$. Let $\Lambda$ be any idele class character of $K^{\times} \backslash A_{K}^{\times}$. Then the degree $10 \mathrm{~L}$ function $L\left(s, \operatorname{ad}(\pi) \times \chi_{K}\right)$ and the degree 20 L-function $L\left(s, \operatorname{ad}(\pi) \times \mathcal{A I}\left(\Lambda^{2}\right)\right)$ both satisfy the usual properties of an L-function, and are both entire.

Proof. It can be verified (as a formal identity that holds at each place) that

$$
L\left(s, \operatorname{Sym}^{2}\left(\pi_{4, K} \times \Lambda\right)\right)=L\left(s, \operatorname{ad}(\pi) \times \mathcal{A} \mathcal{I}\left(\Lambda^{2}\right)\right) .
$$

By Lemma 5.5, $\pi_{4, K} \times \Lambda$ is a cuspidal representation of $\mathrm{GL}_{4}\left(\mathbb{A}_{K}\right)$. Furthermore, if $\pi_{4, K} \times \Lambda$ is self-dual, then it must be symplectic, since $\Pi_{4, K} \times \Lambda$ is the lift of $\pi_{K} \times \Lambda$ from $\operatorname{GSp}_{4}\left(\mathbb{A}_{K}\right)$ to $\mathrm{GL}_{4}\left(\mathbb{A}_{K}\right)$. So by $[9$, Theorem 7.5$], L\left(s, \operatorname{ad}(\pi) \times \mathcal{A I}\left(\Lambda^{2}\right)\right)$ has the properties of an $L$ function and is entire.

To show the same fact for $L\left(s, \operatorname{ad}(\pi) \times \chi_{K}\right)=L\left(s, \operatorname{Sym}^{2}\left(\Pi_{4}\right) \times \chi_{K}\right)$, we appeal to [66, Theorem, p. 104], which shows that the only possible poles of $L\left(s, \operatorname{Sym}^{2}\left(\Pi_{4}\right) \times \chi_{K}\right)$ are at $s=0,1$ (these two possible poles are related by the functional equation). However, by what we have already proved, $L(s, \operatorname{ad}(\pi) \times \mathcal{A} \mathcal{I}(1))=L(s, \operatorname{ad}(\pi)) L\left(s, \operatorname{ad}(\pi) \times \chi_{K}\right)$ is entire. So a pole at $s=1$ for $L\left(s, \operatorname{ad}(\pi) \times \chi_{K}\right)$ would imply that $L(1, \operatorname{ad}(\pi))=0$. This contradicts the last part of Proposition 5.4.

Remark 5.8. Let $F \in S_{k}\left(\Gamma_{0}^{(2)}(N)\right)^{\mathrm{G}}$ give rise to an irreducible representation $\pi$ and assume that $N$ is square-free. Let $d$ be a fundamental discriminant that is divisible by at least one prime not dividing $N$, put $K=\mathbb{Q}(\sqrt{d})$, let $\chi_{d}$ be the quadratic Dirichlet character associated to $K / \mathbb{Q}$, and let $\Lambda$ be a character of the ideal class group $\mathrm{Cl}_{K}$. Then combining Proposition 5.4 and Lemma 5.7, we see that the $L$-functions $L(s, \pi \times \mathcal{A I}(\Lambda))$, $L\left(s, \operatorname{ad}(\pi) \times \mathcal{A I}\left(\Lambda^{2}\right)\right), L\left(s, \operatorname{ad}(\pi) \times \chi_{d}\right), L\left(s, \operatorname{std}(\pi) \times \mathcal{A I}\left(\Lambda^{2}\right)\right)$, and $L\left(s, \operatorname{std}(\pi) \times \chi_{d}\right)$ are all holomorphic everywhere in the complex plane.

\subsection{A consequence of the refined Gan-Gross-Prasad identity}

Let $K=\mathbb{Q}(\sqrt{d})$, where $d<0$ is a fundamental discriminant. Recall from the introduction that for any character $\Lambda$ of the finite group $\mathrm{Cl}_{K}$, we define $B(F, \Lambda)=\sum_{S \in \mathrm{Cl}_{K}} a(F, S) \Lambda(S)$. The refined Gan-Gross-Prasad conjecture in the context of Bessel periods of $\mathrm{PGSp}_{4} \simeq$ $\mathrm{SO}_{5}$ (and more generally, for Bessel periods of orthogonal groups) was formulated in [40, equation (1.1)] and made more explicit in the context of Siegel cusp forms in [15]. This conjecture implies an identity expressing the square of $|B(F, \Lambda)|$ as a ratio of $L$ values, up to some local integrals. For the purpose of this paper, we only need a relatively weak consequence of this identity, which we formulate explicitly as Hypothesis $G$. In this subsection we do not assume that $N$ is square-free.

Suppose that $F \in S_{k}\left(\Gamma_{0}^{(2)}(N)\right)^{\mathrm{T}}$ gives rise to an irreducible representation $\pi$. Then $F$ is said to satisfy Hypothesis $\boldsymbol{G}$ if there exists a constant $C_{F}$ such that for each imaginary 
quadratic field $K=\mathbb{Q}(\sqrt{d})$ (with $d<0$ a fundamental discriminant) and each character $\Lambda$ of $\mathrm{Cl}_{K}$, we have

$$
|B(F, \Lambda)|^{2} \leq C_{F}|d|^{k-1} L\left(\frac{1}{2}, \pi \times \mathcal{A I}(\Lambda)\right)
$$

Proposition 5.9. Let $\pi$ be an irreducible representation that arises from $S_{k}\left(\Gamma_{0}^{(2)}(N)\right)^{\mathrm{T}}$. Suppose that for each factorizable $F \in S_{k}\left(\Gamma_{0}^{(2)}(N)\right)^{\mathrm{T}}$ that gives rise to $\pi$, and each ideal class character $\Lambda$ of an imaginary quadratic field $K=\mathbb{Q}(\sqrt{d})$ (with d a fundamental discriminant), the refined Gan-Gross-Prasad identity (in the form written down in [15, Conjecture 1.3]) holds for $(\phi, \Lambda)$, where $\phi$ is the adelization of $F$. Then any $F \in$ $S_{k}\left(\Gamma_{0}^{(2)}(N)\right)^{\mathrm{T}}$ that gives rise to $\pi$ satisfies Hypothesis $G$.

Proof. Note that the subspace of $S_{k}\left(\Gamma_{0}^{(2)}(N)\right)^{\mathrm{T}}$ generated by forms that give rise to a fixed irreducible representation $\pi$ has a basis consisting of factorizable forms. So for the purpose of verifying Hypothesis G, it suffices to prove the bound (57) for factorizable $F$ whose adelization $\phi=\otimes \phi_{v}$ generates $\pi$. Assuming the truth of [15, Conjecture 1.2] for $(\phi, \Lambda)$, and combining it with the explicit calculations performed in [15, §2.2, §§3.3-3.5], we see that

$$
|B(F, \Lambda)|^{2}=A_{F}|d|^{k-1} L\left(\frac{1}{2}, \pi \times \mathcal{A I}(\Lambda)\right) \prod_{p \mid N_{F}} J_{p}\left(\phi_{p}, \Lambda_{p}\right),
$$

where $A_{F}$ depends on $F, N_{F} \mid N$ is the smallest integer such that $F \in S_{k}\left(\Gamma_{0}^{(2)}\left(N_{F}\right)\right)^{\mathrm{T}}$, and the normalized local factors $J_{p}\left(\phi_{p}, \Lambda_{p}\right)$ (which depend on $p, \phi_{p}, \Lambda_{p}$, and $d$ ) are defined in [15, equation (30)]. To complete the proof, it suffices to show that $J_{p}\left(\phi_{p}, \Lambda_{p}\right) \ll_{p, \phi_{p}} 1$ (i.e., is bounded by some constant that depends on $p$ and $\phi_{p}$ but not on $d$ or $\Lambda_{p}$ ). It suffices to show this for the unnormalized local factors $J_{0, p}\left(\phi_{p}, \Lambda_{p}\right)$ defined in [15, equation (29)], since the normalized local $L$-factors $J_{p}\left(\phi_{p}, \Lambda_{p}\right)$ differ from these only by certain absolutely bounded $L$-factors appearing in [15, equation (30)].

To show this, we move to a purely local setup. Let $p$ be a prime dividing $N_{F}$. Let $F=\mathbb{Q}_{p}$. We fix a set $M$ of coset representatives of $F^{\times} /\left(F^{\times}\right)^{2}$ such that all elements of $M$ are taken from $\mathbb{Z}_{p}$, and each $r \in M$ generates the discriminant ideal of $F(\sqrt{r}) / F$. We let $r_{p}$ equal the unique representative in $M$ that corresponds to $d$. The assumptions imply that

$$
r_{p}=d u_{p}^{2}, \quad \text { for some } u \in\left(\mathbb{Z}_{p}^{\times}\right)^{2}
$$

Let $K$ equal $F \times F$ (the 'split case') if $r_{p} \in F^{2}$ and $K=F\left(\sqrt{r_{p}}\right)=F(\sqrt{d})$ (the 'field case') if $r_{p} \notin F^{2}$. Fix the matrix $S=S_{d}$ as in [15, (75)], so that $S_{d}$ has discriminant $d$. Let $T(F)=T_{S}(F) \simeq K^{\times}$be the associated subgroup of $\operatorname{GSp}_{4}(F)$ and let $N(F) \subset \operatorname{GSp}_{4}(F)$ denote the unipotent for the Siegel parabolic and $\theta_{S}$ the character on $N(F)$ given by $\theta_{S}\left(\left(\begin{array}{cc}I_{2} & X \\ 0 & I_{2}\end{array}\right)\right)=\psi(\operatorname{tr}(S X))$, where $\psi$ is a fixed unramified additive character. Then we 
need to show that the integral

$$
J_{0, p}\left(\phi_{p}, \Lambda_{p}\right)=\int_{F^{\times} \backslash T(F)} \int_{N(F)}^{\mathrm{St}}\left\langle\pi_{p}\left(t_{p} n_{p}\right) \phi_{p}, \phi_{p}\right\rangle \Lambda_{p}^{-1}\left(t_{p}\right) \theta_{S}^{-1}\left(n_{p}\right) d n_{p} d t_{p}
$$

is bounded by some quantity that does not depend on $d$ or $\Lambda_{p}$. Note that the superscript 'St' denotes a stable integral as in [40]; this means that the integral can be replaced by any sufficiently large compact subgroup (as we will do later). Put $S^{\prime}=\left(\begin{array}{cc}-\frac{r_{p}}{4} & 0 \\ 0 & 1\end{array}\right), A^{\prime}=$ $\left(\begin{array}{cc}u_{p} & 0 \\ 0 & 1\end{array}\right)$ if $d \equiv 0(\bmod 4)$. In the case where $d \equiv 1(\bmod 4)$, we put $S^{\prime}=\left(\begin{array}{cc}\frac{1-r_{p}}{4} & \frac{1}{2} \\ \frac{1}{2} & 1\end{array}\right), A^{\prime}=$ $\left(\begin{array}{cc}u_{p} & 0 \\ \frac{1-u_{p}}{2} & 1\end{array}\right)$. In either case, $A^{\prime} \in \mathrm{GL}_{2}\left(\mathbb{Z}_{p}\right)$ and $S^{\prime}={ }^{t} A S A$. To show that $J_{p}$ is bounded independently of $d$, we use a simple change of variables $\left(n_{p} \mapsto A n_{p}{ }^{t} A, t_{p} \mapsto A t_{p}^{\prime} A^{-1}\right)$ to see that the integral $J_{0, p}\left(\phi_{p}, \Lambda_{p}\right)$ remains unchanged when the matrix $S$ is replaced by $S^{\prime}$. This shows that $J_{0, p}\left(\phi_{p}, \Lambda_{p}\right)$ depends not on the actual value of $d$ but only on the class of $d$ in $F^{\times} /\left(F^{\times}\right)^{2}$ together with $d$ modulo 4 , of which there are only finitely many possibilities. To show that the resulting integral is absolutely bounded independently of $\Lambda$, we replace the integral $\int_{N(F)}^{\mathrm{St}}$ by $\int_{p^{-m_{p}} N\left(\mathbb{Z}_{p}\right)}$, where $m_{p}$ depends only on the level $N$ (as follows from the argument of [15, Proposition 2.14]). The resulting integral is absolutely convergent [40, Theorem 2.1(i)] and hence bounded independently of $\Lambda$.

Remark 5.10. Furusawa and Morimoto [18] have proved the refined Gan-Gross-Prasad identity in the form required in Proposition 5.9 for $\Lambda=1_{K}$, and they have announced a proof of this identity for general $\Lambda$.

Remark 5.11. Under certain assumptions (namely $N$ odd and square-free, $F$ a newform, and $\left(\frac{d}{p}\right)=-1$ for all primes $p$ dividing $\left.N\right)$, the relevant local integrals were explicitly computed in [15], and so under these assumptions Proposition 5.9 follows from [15, Theorem 1.13].

Corollary 5.12. Suppose that $F \in S_{k}\left(\Gamma_{0}^{(2)}(N)\right)^{\mathrm{Y}}$ gives rise to an irreducible representation. Then $F$ satisfies Hypothesis $G$.

Proof. This follows from Proposition 5.9, since the refined Gan-Gross-Prasad conjecture is known for Yoshida lifts by [40, Theorem 4.3].

Proof of Corollary 1.1. Let $\pi$ be a cuspidal automorphic representation of $\mathrm{GSp}_{4}(\mathbb{A})$ that arises from a form $F \in S_{k}\left(\Gamma_{0}^{(2)}(N)\right)^{\mathrm{T}}$ with $k>2$ even and $N$ odd and square-free. Assume that the refined Gan-Gross-Prasad identity holds. Then for any fundamental matrix $S \in \Lambda_{2}$ of discriminant $d$, we put $K=\mathbb{Q}(\sqrt{d})$ and use Cauchy-Schwarz and Proposition 5.9 to conclude 


$$
\begin{aligned}
|a(F, S)|^{2} & =\frac{1}{\left|\mathrm{Cl}_{K}\right|^{2}}\left|\sum_{\Lambda \in \widehat{\mathrm{Cl}_{K}}} B(F, \Lambda) \Lambda^{-1}(S)\right|^{2} \leq \frac{1}{\left|\mathrm{Cl}_{K}\right|} \sum_{\Lambda \in \widehat{\mathrm{Cl}_{K}}}|B(F, \Lambda)|^{2} \\
& \ll_{\pi}|d|^{k-1} \frac{1}{\left|\mathrm{Cl}_{K}\right|} \sum_{\Lambda \in \widehat{\mathrm{Cl}_{K}}} L\left(\frac{1}{2}, \pi \times \mathcal{A I}(\Lambda)\right) .
\end{aligned}
$$

The proof of the corollary now follows from Theorem B.

Proof of Corollary 1.2. For $f, g$ as in Corollary 1.2 and $N=\operatorname{lcm}\left(N_{1}, N_{2}\right)$, there exists $F \in S_{k}\left(\Gamma_{0}^{(2)}(N)\right)^{\mathrm{Y}}$ which gives rise to an irreducible representation $\pi$ such that (see [58, Proposition 3.1])

$$
L(s, \pi \times \mathcal{A} \mathcal{I}(\Lambda))=L(s, f \times \mathcal{A I}(\Lambda)) L(s, g \times \mathcal{A I}(\Lambda)) .
$$

Now Corollary 1.2 follows from Corollary 1.1 and the triangle inequality.

Proposition 5.13. Suppose that $F \in S_{k}\left(\Gamma_{0}^{(2)}(N)\right)^{\mathrm{CAP}}$ gives rise to an irreducible representation $\pi$ such that $L^{N}(s, \pi)=L^{N}\left(s, \pi_{0}\right) \zeta^{N}\left(s+\frac{1}{2}\right) \zeta^{N}\left(s-\frac{1}{2}\right)$, where $\pi_{0}$ is a representation of $\mathrm{GL}_{2}(\mathbb{A})$. Then the Fourier coefficients of $F$ at fundamental matrices depend on only the associated discriminant - that is, if $S_{1}, S_{2}$ are two fundamental matrices with the same discriminant $d$, then $a\left(F, S_{1}\right)=a\left(F, S_{2}\right)$. Furthermore, there exists a constant $C_{F}$ such that for each fundamental discriminant $d$ and any matrix $S$ of discriminant $d$, we have

$$
|a(F, S)| \leq C_{F}|d|^{\frac{k}{2}-\frac{3}{4}} L\left(\frac{1}{2}, \pi_{0} \times \chi_{d}\right)^{1 / 2} .
$$

Proof. An analogue of the Gan-Gross-Prasad conjecture for representations of SaitoKurokawa type was formulated and proved by Qiu in [49]. In particular, we have $B(F, \Lambda)=$ 0 unless $\Lambda$ is the trivial character, which proves the assertion that the Fourier coefficients of $F$ at fundamental matrices depend on only the discriminant. For $\Lambda$ trivial, the desired inequality follows in a similar fashion as in the proof of Proposition 5.9.

\subsection{Proof of Theorem C}

In this subsection we restate and prove Theorem C, assuming the main result of $\S 6$.

Theorem 5.14. Let $k>2$ be even and $N \geq 1$ be square-free.

i) Assume that the GRH holds for each of the L-functions $L(s, \pi \times \mathcal{A I}(\Lambda))$, $L\left(s, \operatorname{ad}(\pi) \times \mathcal{A I}\left(\Lambda^{2}\right)\right), L\left(s, \operatorname{ad}(\pi) \times \chi_{d}\right), L\left(s, \operatorname{std}(\pi) \times \mathcal{A I}\left(\Lambda^{2}\right)\right), L\left(s, \operatorname{std}(\pi) \times \chi_{d}\right)$, $L\left(s, \sigma \times \chi_{d}\right)$, and $L\left(s, \chi_{d}\right)$, where $\pi$ is any automorphic representation of $\operatorname{GSp}_{4}(\mathbb{A})$ that arises from $S_{k}\left(\Gamma_{0}^{(2)}(N)\right)^{\mathrm{G}}$, $\sigma$ is any cuspidal automorphic representation of $\mathrm{GL}_{2}(\mathbb{A}), K=\mathbb{Q}(\sqrt{d})$ is an imaginary quadratic field with associated Dirichlet character $\chi_{d}$, and $\Lambda$ is an ideal class character of $K$.

ii) Assume that each $F^{\prime} \in S_{k}\left(\Gamma_{0}^{(2)}(N)\right)^{\mathrm{G}}$ that gives rise to an irreducible representation satisfies Hypothesis $G$ (by Proposition 5.9, this is implied by the refined Gan-GrossPrasad conjecture). 
Then given any $F \in S_{k}\left(\Gamma_{0}^{(2)}(N)\right)$, we have, for fundamental matrices $S$,

$$
|a(F, S)| \ll_{F, \varepsilon} \frac{|\operatorname{disc}(S)|^{\frac{k}{2}-\frac{1}{2}}}{(\log |\operatorname{disc}(S)|)^{\frac{1}{8}-\varepsilon}} .
$$

Remark 5.15. Concerning the automorphy of the various representations that appear in the statement of Theorem 5.14, we refer the reader to the statements and proofs of Proposition 5.4 and Lemma 5.7.

Proof. Since $S_{k}\left(\Gamma_{0}^{(2)}(N)\right)$ has a basis consisting of forms that give rise to irreducible representations, it suffices to prove the bound (58) for such forms F. Furthermore, we may assume that $d=\operatorname{disc}(S)$ is divisible by at least one prime not dividing $N$, since there are at most finitely many fundamental $S$ without this property. Let $F$ give rise to $\pi$. We consider three cases, corresponding to decomposition (53).

Case 1: $\pi$ is of Saito-Kurokawa type. In this case, using Proposition 5.13, the desired inequality follows from any subconvexity bound for $L\left(\frac{1}{2}, \pi_{0} \times \chi_{d}\right)$, which is known. (In particular, for this case, we do not need to appeal to GRH for $\left.L\left(s, \pi_{0} \times \chi_{d}\right)\right)$.

Case 2: $\pi$ is of Yoshida type - that is, $F \in S_{k}\left(\Gamma_{0}^{(2)}(N)\right)^{\mathrm{Y}}$. In this case, there are inequivalent representations $\sigma_{1}$ and $\sigma_{2}$ of $\mathrm{GL}_{2}(\mathbb{A})$ such that $L\left(\frac{1}{2}, \pi \times \mathcal{A I}(\Lambda)\right)=$ $L\left(\frac{1}{2}, \sigma_{1} \times \mathcal{A I}(\Lambda)\right) L\left(\frac{1}{2}, \sigma_{2} \times \mathcal{A I}(\Lambda)\right)$. Using the identity $a(F, S)=\frac{1}{\left|\mathrm{Cl}_{K}\right|} \sum_{\Lambda \in \widehat{\mathrm{Cl}_{K}}} B(F, \Lambda)$ $\Lambda^{-1}(S)$ and Corollary 5.12, we conclude that

$$
a(F, S) \ll_{F}|d|^{\frac{k}{2}-\frac{1}{2}} \frac{1}{\left|\mathrm{Cl}_{K}\right|} \sum_{\Lambda \in \widehat{\mathrm{Cl}_{K}}} \sqrt{L\left(\frac{1}{2}, \sigma_{1} \times \mathcal{A I}(\Lambda)\right) L\left(\frac{1}{2}, \sigma_{2} \times \mathcal{A I}(\Lambda)\right)} .
$$

Now the desired conclusion follows from [5, Theorem 3].

Case 3: $\pi$ is of general type - that is, $F \in S_{k}\left(\Gamma_{0}^{(2)}(N)\right)^{\mathrm{G}}$. In this case, using $a(F, S)=$ $\frac{1}{\left|\mathrm{Cl}_{K}\right|} \sum_{\Lambda \in \widehat{\mathrm{Cl}_{K}}} B(F, \Lambda) \Lambda^{-1}(S)$ and Hypothesis $\mathrm{G}$, we get

$$
a(F, S) \ll_{F}|d|^{\frac{k}{2}-\frac{1}{2}} \frac{1}{\left|\mathrm{Cl}_{K}\right|} \sum_{\Lambda \in \widehat{\mathrm{Cl}_{K}}} \sqrt{L\left(\frac{1}{2}, \pi \times \mathcal{A I}(\Lambda)\right)} .
$$

Now the desired conclusion follows from Theorem 6.1, which will be proved in the next section.

\section{Fractional moments of central $L$-values}

\subsection{Statement of result}

Throughout this section, we let $\pi$ denote an irreducible cuspidal automorphic representation of $\mathrm{GSp}_{4}(\mathbb{A})$ that arises from $S_{k}\left(\Gamma_{0}(N)\right)^{\mathrm{G}}$ (see $\left.\S 5.4\right)$. Assume that $N \geq 1$ is squarefree and $k \geq 2$. Let $K=\mathbb{Q}(\sqrt{d})$ be a quadratic field such that $d<0$ is a fundamental discriminant divisible by at least one prime not dividing $N$, and let $\mathrm{Cl}_{K}$ denote the ideal class group of $K$. Also, given $\Lambda \in \widehat{\mathrm{Cl}_{K}}$, we let $\mathcal{A I}(\Lambda)$ denote the automorphic representation of $\mathrm{GL}_{2}(\mathbb{A})$ given by automorphic induction; it is generated by the theta series $\Theta_{\Lambda}(z)=\sum_{0 \neq \mathfrak{a} \subset \mathcal{O}_{K}} \Lambda(\mathfrak{a}) e(N(\mathfrak{a}) z)$. Our assumptions imply that the $L$-functions $L(s, \pi \times \mathcal{A} \mathcal{I}(\Lambda)), L\left(s, \operatorname{ad}(\pi) \times \mathcal{A I}\left(\Lambda^{2}\right)\right), \quad L\left(s, \operatorname{ad}(\pi) \times \chi_{d}\right), \quad L\left(s, \operatorname{std}(\pi) \times \mathcal{A I}\left(\Lambda^{2}\right)\right)$, and 
$L\left(s, \operatorname{std}(\pi) \times \chi_{d}\right)$ are all entire (see Remark 5.8). Using Soundararajan's method [64] for bounding moments of $L$-functions, we will prove the following result:

Theorem 6.1. Assume the GRH for each of the L-functions $L(s, \pi \times \mathcal{A I}(\Lambda))$, $L\left(s, \operatorname{ad}(\pi) \times \mathcal{A} \mathcal{I}\left(\Lambda^{2}\right)\right), L\left(s, \operatorname{ad}(\pi) \times \chi_{d}\right), L\left(s, \operatorname{std}(\pi) \times \mathcal{A I}\left(\Lambda^{2}\right)\right), L\left(s, \operatorname{std}(\pi) \times \chi_{d}\right), \quad$ and $L\left(s, \chi_{d}\right)$. Then

$$
\frac{1}{\left|\mathrm{Cl}_{K}\right|} \sum_{\Lambda \in \widehat{\mathrm{Cl}_{K}}} \sqrt{L\left(\frac{1}{2}, \pi \times \mathcal{A I}(\Lambda)\right)} \ll_{\pi, \varepsilon} \frac{1}{(\log |d|)^{\frac{1}{8}-\varepsilon}}
$$

Related moment estimates for families of $L$-functions have been established in $[5,27$, $38,43]$. Since we assume the GRH, we immediately get that $L\left(\frac{1}{2}, \pi \times \mathcal{A I}(\Lambda)\right) \geq 0$, which incidentally also follows from the refined Gan-Gross-Prasad identity using formula (57). However, the nonnegativity of $L\left(\frac{1}{2}, \pi \times \mathcal{A I}(\Lambda)\right)$ is known unconditionally due to [37, Theorem 1.1], so the square root of the central value is unambiguous, independent of the truth of any unproven hypothesis.

\subsection{Local computation}

For $\operatorname{Re}(s)>1$, the $L$-function $L(s, \pi)$ is given by

$L(s, \pi)=\prod_{p} L\left(s, \pi_{p}\right), \quad L\left(s, \pi_{p}\right)=\left(1-\frac{\alpha_{p}}{p^{s}}\right)^{-1}\left(1-\frac{\alpha_{p}^{-1}}{p^{s}}\right)^{-1}\left(1-\frac{\beta_{p}}{p^{s}}\right)^{-1}\left(1-\frac{\beta_{p}^{-1}}{p^{s}}\right)^{-1}$,

for $p \nmid N$, where $\mathcal{S}_{p}(\pi)=\left\{\alpha_{p}, \alpha_{p}^{-1}, \beta_{p}, \beta_{p}^{-1}\right\}$ are the Satake parameters of $\pi$ and it is known that $\left|\alpha_{p}\right|=\left|\beta_{p}\right|=1$ by [68]. Also, given $\Lambda \in \widehat{\mathrm{Cl}_{K}}$, we have, for $\operatorname{Re}(s)>1$,

$$
L(s, \mathcal{A} \mathcal{I}(\Lambda))=\prod_{p} L\left(s, \mathcal{A I}\left(\Lambda_{p}\right)\right), \quad L\left(s, \mathcal{A I}\left(\Lambda_{p}\right)\right)=\left(1-\frac{\alpha_{\Lambda_{p}}}{p^{s}}\right)^{-1}\left(1-\frac{\beta_{\Lambda_{p}}}{p^{s}}\right)^{-1},
$$

for $p \nmid N$, where the Satake parameters $\mathcal{S}_{p}(\mathcal{A I}(\Lambda))=\left\{\alpha_{\Lambda_{p}}, \beta_{\Lambda_{p}}\right\}$ satisfy the following:

- If $\left(\frac{d}{p}\right)=1$, then $p \mathcal{O}_{k}=\mathfrak{p} \overline{\mathfrak{p}}$ and $\alpha_{\Lambda_{p}}=\Lambda(\mathfrak{p}), \beta_{\Lambda_{p}}=\Lambda(\overline{\mathfrak{p}})=\overline{\alpha_{\Lambda_{p}}}$.

- If $\left(\frac{d}{p}\right)=-1$, then $\alpha_{\Lambda_{p}}=1$ and $\beta_{\Lambda_{p}}=-1$.

- If $p \mid d$, then $p \mathcal{O}_{k}=\mathfrak{p}^{2}$ and $\alpha_{\Lambda_{p}}=\Lambda(\mathfrak{p}), \beta_{\Lambda_{p}}=0$.

In this notation, we have that the Satake parameters of $L(s, \operatorname{std}(\pi))$ and $L(s, \operatorname{ad}(\pi))$ are respectively given by

$$
\begin{aligned}
\mathcal{S}_{p}(\operatorname{std}(\pi)) & =\left\{1, \alpha_{p} \beta_{p}, \alpha_{p} \beta_{p}^{-1}, \alpha_{p}^{-1} \beta_{p}, \alpha_{p}^{-1} \beta_{p}^{-1}\right\} \\
\mathcal{S}_{p}(\operatorname{ad}(\pi)) & =\left\{\alpha_{p}^{2}, \alpha_{p}^{-2}, \beta_{p}^{2}, \beta_{p}^{-2}, \alpha_{p} \beta_{p}, \alpha_{p}^{-1} \beta_{p}, \alpha_{p} \beta_{p}^{-1}, \alpha_{p}^{-1} \beta_{p}^{-1}, 1,1\right\} .
\end{aligned}
$$

For $\star \in\{\pi, \mathcal{A} \mathcal{I}(\Lambda), \operatorname{std}(\pi), \operatorname{ad}(\pi)\}$ and $p \nmid N$, define

$$
a_{\star}\left(p^{n}\right)=\sum_{\alpha \in \mathcal{S}_{p}(\star)} \alpha^{n} .
$$


Also, for $f, g \in\{\pi, \mathcal{A I}(\Lambda), \operatorname{std}(\pi), \operatorname{ad}(\pi)\}$, let $a_{f \times g}\left(p^{n}\right)=a_{f}\left(p^{n}\right) a_{g}\left(p^{n}\right)$ and $a_{f \times \chi_{d}}\left(p^{n}\right)=$ $a_{f}\left(p^{n}\right) \chi_{d}\left(p^{n}\right)$.

We now make the following observations. First, using $\left|\alpha_{p}\right|=\left|\beta_{p}\right|=1$, it immediately follows that for any $n \geq 1$ and $p \nmid N$,

$$
\left|a_{\pi \times \mathcal{A} \mathcal{I}(\Lambda)}\left(p^{n}\right)\right| \leq 8 .
$$

Additionally, observe for $p \nmid N$ that

$$
a_{\mathrm{ad}(\pi)}(p)=\alpha_{p}^{2}+\alpha_{p}^{-2}+\beta_{p}^{2}+\beta_{p}^{-2}+a_{\mathrm{std}(\pi)}(p)+1 .
$$

Hence, for $p \nmid N$,

$$
a_{\pi}\left(p^{2}\right)=a_{\mathrm{ad}(\pi)}(p)-a_{\mathrm{std}(\pi)}(p)-1
$$

Also for $p \nmid N$,

$$
\begin{aligned}
a_{\pi}(p)^{2} & =\alpha_{p}^{2}+\alpha_{p}^{-2}+\beta_{p}^{2}+\beta_{p}^{-2}+2\left(\alpha_{p}+\alpha_{p}^{-1}\right)\left(\beta_{p}+\beta_{p}^{-1}\right)+4 \\
& =a_{\mathrm{ad}(\pi)}(p)+a_{\operatorname{std}(\pi)}(p)+1 .
\end{aligned}
$$

Recall that for $\left(\frac{d}{p}\right) \neq-1$, we have $\alpha_{\Lambda_{p}}^{2}=\Lambda^{2}(\mathfrak{p})=\alpha_{\Lambda_{p}^{2}}$. Thus, we get that for $p \nmid N$,

$$
a_{\mathcal{A I}(\Lambda)}\left(p^{2}\right)=a_{\mathcal{A I}\left(\Lambda^{2}\right)}(p)+\left(\frac{d}{p}\right)^{2}-\left(\frac{d}{p}\right)= \begin{cases}\alpha_{\Lambda_{p}^{2}}+\beta_{\Lambda_{p}^{2}} & \text { if }\left(\frac{d}{p}\right) \neq-1 \\ 2 & \text { if }\left(\frac{d}{p}\right)=-1\end{cases}
$$

Therefore, combining this equation with equation (60), we conclude that

$$
a_{\pi \times \mathcal{A} \mathcal{I}(\Lambda)}\left(p^{2}\right)=\left(a_{\mathrm{ad}(\pi)}(p)-a_{\mathrm{std}(\pi)}(p)-1\right)\left(a_{\mathcal{A I}\left(\Lambda^{2}\right)}(p)+\left(\frac{d}{p}\right)^{2}-\left(\frac{d}{p}\right)\right) .
$$

\subsection{Preliminary lemmas}

Soundararajan's method for moments of $L$-functions starts with a remarkable bound for the central $L$-value given in terms of a Dirichlet polynomial supported on prime powers. This inequality has been generalized by Chandee [11].

Lemma 6.2. Assume the $G R H$ for $L(s, \pi \times \mathcal{A I}(\Lambda))$. There exists $C_{0}>1$, which depends at most on $N, k$ such that for $x \geq 2$,

$$
\log L\left(\frac{1}{2}, \pi \times \mathcal{A I}(\Lambda)\right) \leq \sum_{\substack{p^{n} \leq x \\ p \nmid N}} \frac{a_{\pi \times \mathcal{A} \mathcal{I}(\Lambda)}\left(p^{n}\right)}{n p^{\frac{n}{2}(1+1 / \log x)}}+\frac{C_{0} \log |d|}{\log x} .
$$

Remark 6.3. Taking $x=\log |d|$ and using the bound (59), it follows that

$$
L\left(\frac{1}{2}, \pi \times \mathcal{A} \mathcal{I}(\Lambda)\right) \ll \exp \left(2 C_{0} \frac{\log |d|}{\log \log |d|}\right) .
$$

Proof. This follows immediately from [11, Theorem 2.1]. 
To analyze the foregoing sum over prime powers, we will separately consider the primes, squares of primes, and higher prime powers. The contribution of the squares of primes is estimated in the following result:

Lemma 6.4. Assume the GRH. Then for $x \geq 2$, the following hold:

i) $\sum_{\substack{p \leq \sqrt{x} \\ p \nmid N}} \frac{a_{\pi \times \mathcal{A} \mathcal{I}(\Lambda)}\left(p^{2}\right)}{p^{1+2 / \log x}} \frac{\log x / p}{\log x}=-\log \log x(1+o(1))+O_{\pi}(\log \log \log |d|)$ and

ii) $\sum_{\substack{p \leq x \\ p \nmid N,\left(\frac{d}{p}\right)=1}} \frac{a_{\pi}(p)^{2}}{p}=\frac{1}{2} \log \log x(1+o(1))+O_{\pi}(\log \log \log |d|)$.

Remark 6.5. We assume that the GRH holds for $L\left(s, \chi_{d}\right), L(s, \pi \times \mathcal{A I}(\Lambda))$, $L\left(s, \operatorname{ad}(\pi) \times \mathcal{A I}\left(\Lambda^{2}\right)\right), L\left(s, \operatorname{ad}(\pi) \times \chi_{d}\right), L\left(s, \operatorname{std}(\pi) \times \mathcal{A I}\left(\Lambda^{2}\right)\right)$, and $L\left(s, \operatorname{std}(\pi) \times \chi_{d}\right)$.

Proof. Using $\left|\alpha_{p}\right|=\left|\beta_{p}\right|=1$, we get, for $x \geq(\log |d|)^{3}$,

$$
\sum_{\substack{p \leq x \\ p \nmid N}} \frac{a_{\operatorname{std}(\pi) \times \chi_{d}}(p)}{p}=\sum_{\substack{(\log |d|)^{3} \leq p \leq x \\ p \nmid N}} \frac{a_{\operatorname{std}(\pi) \times \chi_{d}}(p)}{p}+O(\log \log \log |d|) .
$$

By Proposition $5.4, L\left(s, \operatorname{std}(\pi) \times \chi_{d}\right)$ belongs to the extended Selberg class, so that we may use [5, Lemma 5]. Applying this lemma twice, it follows that the sum on the right-hand side is $O_{\pi}(1)$, and so

$$
\sum_{\substack{p \leq x \\ p \nmid N}} \frac{a_{\operatorname{std}(\pi)}(p)\left(\frac{d}{p}\right)}{p} \ll_{\pi} \log \log \log |d| .
$$

Repeating the foregoing above and recalling Remark 5.8, it follows that for $\star \in\left\{\operatorname{ad}(\pi) \times \chi_{d}, \operatorname{ad}(\pi) \times \mathcal{A I}\left(\Lambda^{2}\right), \operatorname{std}(\pi) \times \mathcal{A I}\left(\Lambda^{2}\right), \mathcal{A I}\left(\Lambda^{2}\right)\right\}$, we have $\sum_{\substack{p \leq x \\ p \nmid N}} \frac{a_{\star}(p)}{p} \ll$ $\log \log \log |d|$.Also, $\sum_{p \mid d} \frac{1}{p} \ll \log \log \log |d|$, and under the GRH we have $\sum_{p \leq x} \frac{\chi_{d}(p)}{p} \ll$ $\log \log \log |d|$. Finally, using the fact that $L(s, \operatorname{std}(\pi))$ and $L(s, \operatorname{ad}(\pi))$ do not vanish on the line $\operatorname{Re}(s)=1$ - the latter of which follows from Proposition 5.4 - we have that (see, e.g., [31, Theorem 1])

$$
\sum_{\substack{p \leq x \\ p \nmid N}} \frac{a_{\mathrm{ad}(\pi)}(p)}{p}=o(\log \log x), \quad \sum_{\substack{p \leq x \\ p \nmid N}} \frac{a_{\mathrm{std}(\pi)}(p)}{p}=o(\log \log x) .
$$

Therefore, applying equation (62) and using the foregoing estimates along with partial summation to handle the smooth weight $p^{-2 / \log x} \frac{\log x / p}{\log x}$ completes the proof of (i).

To prove (ii), we rewrite the condition $\left(\frac{d}{p}\right)=1$ as $\frac{1}{2}\left(\left(\frac{d}{p}\right)+1\right)$ for $p \nmid d$, use equation (61) and argue as before. We trivially bound the contribution of $p \mid d$ by $\leq 16 \sum_{p \mid d} \frac{1}{p} \ll$ $\log \log \log |d|$. 


\subsection{Large deviations of Dirichlet polynomials}

To bound the frequency with which large values of $\log L\left(\frac{1}{2}, \pi \times \mathcal{A I}(\Lambda)\right)$ occur, we will estimate large deviations of the sum over primes in Lemma 6.2 by bounding its moments. This is done in the following fairly standard lemma (e.g. [64, Lemma 3]), whose proof we include for completeness:

Lemma 6.6. Let $\left\{b_{p}\right\}_{p} \subset \mathbb{R}$ and $\ell \in \mathbb{N}$. Suppose $x^{\ell}<\sqrt{|d|} / 2$. Then the following hold:
i) $\frac{1}{\left|\mathrm{Cl}_{K}\right|} \sum_{\Lambda \in \widehat{\mathrm{Cl}_{K}}}\left(\sum_{\substack{p \leq x \\ p \nmid d, p \nmid N}} \frac{b_{p} a_{\mathcal{A I}(\Lambda)}(p)}{\sqrt{p}}\right)^{2 \ell} \leq \frac{(2 \ell) !}{2^{\ell} \ell !}\left(2 \sum_{\substack{p \leq x \\\left(\frac{d}{p}\right)=1, p \nmid N}} \frac{b_{p}^{2}}{p}\right)^{\ell}$ and
ii) $\frac{1}{\left|\mathrm{Cl}_{K}\right|} \sum_{\Lambda \in \widehat{\mathrm{Cl}} K}\left(\sum_{\substack{p \leq x \\ p \mid d, p \nmid N}} \frac{b_{p} a_{\mathcal{A I}(\Lambda)}(p)}{\sqrt{p}}\right)^{2 \ell} \leq \frac{(2 \ell) !}{2^{\ell} \ell !}\left(\sum_{\substack{p \leq x \\ p \mid d, p \nmid N}} \frac{b_{p}^{2}}{p}\right)^{\ell}$.

Proof. Let $b_{n}=\prod_{p^{j} \| n} b_{p}^{j}$. We have

$$
\begin{aligned}
& \frac{1}{\left|\mathrm{Cl}_{K}\right|} \sum_{\Lambda \in \widehat{\mathrm{Cl}_{K}}}\left(\sum_{\substack{p \leq x \\
p \nmid d, p \nmid N}} \frac{b_{p} a_{\mathcal{A I}(\Lambda)}(p)}{\sqrt{p}}\right)^{2 \ell} \\
& =\sum_{\substack{n \geq 1 \\
(n, N)=1}} \frac{b_{n}}{\sqrt{n}} \sum_{\substack{\mathfrak{p}_{1}, \ldots, \mathfrak{p}_{2 \ell} \subset \mathcal{O}_{K} \\
N\left(\mathfrak{p}_{1} \cdots \mathfrak{p}_{2 \ell}\right)=n \\
N\left(\mathfrak{p}_{j}\right) \leq x \\
\mathfrak{p}_{j} \text { split }}} \frac{1}{\left|\mathrm{Cl}_{K}\right|} \sum_{\Lambda \in \widehat{\mathrm{Cl}} K} \prod_{j=1}^{2 \ell}\left(\Lambda\left(\mathfrak{p}_{j}\right)+\Lambda\left(\mathfrak{p}_{j}\right)^{-1}\right) .
\end{aligned}
$$

For $n \in \mathbb{N}$ in this sum, write $n=q_{1}^{e_{1}} \cdots q_{r}^{e_{r}}$, where $q_{1}, \ldots, q_{r}$ are distinct primes with $q_{j} \mathcal{O}_{K}=\mathfrak{q}_{j} \overline{\mathfrak{q}_{j}}$ for each $j=1, \ldots, r$. The inner sum on the right-hand side equals

$$
\sum_{0 \leq f_{1} \leq e_{1}} \cdots \sum_{0 \leq f_{r} \leq e_{r}}\left(\begin{array}{c}
e_{1} \\
f_{1}
\end{array}\right) \cdots\left(\begin{array}{c}
e_{r} \\
f_{r}
\end{array}\right) \frac{1}{\left|\mathrm{Cl}_{K}\right|} \sum_{\Lambda \in \widehat{\mathrm{Cl}_{K}}} \Lambda\left(\mathfrak{q}_{1}^{f_{1}} \overline{\mathfrak{q}}_{1}^{e_{1}-f_{1}} \cdots \mathfrak{q}_{r}^{f_{r}}{\overline{\mathfrak{q}_{r}}}^{e_{r}-f_{r}}\right) .
$$

Write $\mathfrak{a}=\mathfrak{q}_{1}^{f_{1}} \overline{\mathfrak{q}}_{1} e_{1}-f_{1} \cdots \mathfrak{q}_{r}^{f_{r}} \overline{\mathfrak{q}}_{r} e_{r}-f_{r}$. Since $N(\mathfrak{a}) \leq x^{2 \ell}<|d| / 4$, the inner sum is zero unless $\mathfrak{a}=(\alpha)$ and $\alpha \in \mathbb{Z}$. Hence the only terms that do not vanish are those with $e_{1}, \ldots, e_{r}$ even and $f_{j}=e_{j} / 2$ for $j=1, \ldots, r$. Define the multiplicative functions $\nu(n)$ by $\nu\left(p^{a}\right)=1 / a$ ! and $g(n)$ by $g\left(p^{a}\right)=\left(\begin{array}{c}a \\ a / 2\end{array}\right)$ if $2 \mid a$ and $g\left(p^{a}\right)=0$ otherwise. The right-hand side of equation (63) equals

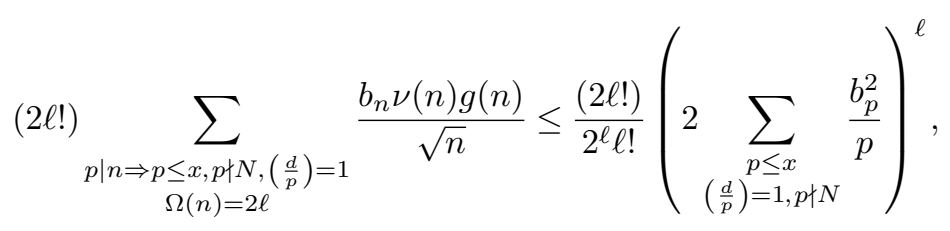


where for the last inequality we used the facts that the sum on the left-hand side is supported on squares and $\nu\left(m^{2}\right) g\left(m^{2}\right) \leq \nu(m)$. This completes the proof of (i).

Similarly, we have

$$
\frac{1}{\left|\mathrm{Cl}_{K}\right|} \sum_{\Lambda \in \widehat{C l_{K}}}\left(\sum_{\substack{p \leq x \\ p \mid d, p \nmid N}} \frac{b_{p} a_{\mathcal{A I}(\Lambda)}(p)}{\sqrt{p}}\right)^{2 \ell}=\sum_{n \geq 1} \frac{b_{n}}{\sqrt{n}} \sum_{\substack{\mathfrak{p}_{1}, \ldots, \mathfrak{p}_{2 \ell} \subset \mathcal{O}_{K} \\ N\left(\mathfrak{p}_{1} \cdots \mathfrak{p}_{2 \ell}\right)=n \\ N\left(\mathfrak{p}_{j}\right) \leq x \\ \mathfrak{p}_{j} \text { ramified }}} \frac{1}{\left|\mathrm{Cl}_{K}\right|} \sum_{\Lambda \in \widehat{\mathrm{Cl}_{K}}} \Lambda\left(\mathfrak{p}_{1} \cdots \mathfrak{p}_{2 \ell}\right) .
$$

Write $\mathfrak{a}=\mathfrak{p}_{1} \cdots \mathfrak{p}_{2 \ell}$. The inner sum is zero unless $\mathfrak{a}=(\alpha)$ with $\alpha \in \mathbb{Z}$, since $N(\mathfrak{a})<|d| / 4$. Consequently, $n$ is a square. The right-hand side equals

$$
(2 \ell) ! \sum_{\substack{p|n \Rightarrow p \leq x, p \nmid N, p| d \\ \Omega(n)=\ell}} \frac{b_{n}^{2}}{n} \nu\left(n^{2}\right) \leq \frac{(2 \ell) !}{2^{\ell} \ell !}\left(\sum_{\substack{p \leq x \\ p \mid d, p \nmid N}} \frac{b_{p}^{2}}{p}\right)^{\ell},
$$

where we used the fact that $\nu\left(n^{2}\right) \leq \frac{\nu(n)}{2^{\Omega(n)}}$ in the last step. This completes the proof of (ii).

$$
\begin{aligned}
& \text { For } x \geq 2 \text {, let } P(\Lambda ; x)=\sum_{\substack{p \leq x \\
p \nmid N}} \frac{a_{\pi}(p) a_{\mathcal{A I}(\Lambda)}(p)}{p^{\frac{1}{2}+\frac{1}{\log x}}} \frac{\log x / p}{\log x} \text {, and for } V \in \mathbb{R} \text { let } A_{K}(V ; x)= \\
& \frac{1}{\left|\mathrm{Cl}_{K}\right|}\left|\left\{\Lambda \in \widehat{\mathrm{Cl}}_{K}: P(\Lambda ; x)>V\right\}\right| \text {. }
\end{aligned}
$$

Lemma 6.7. Let $\epsilon>0$ be sufficiently small. Then for $(\log \log |d|)^{3 / 4} \leq V \leq \frac{4 C_{0} \log |d|}{\log \log |d|}$, we have

$$
A_{K}\left(V ;|d|^{\frac{1}{\epsilon V}}\right) \ll_{\pi, \epsilon} \exp \left(\frac{-V^{2}}{2 \log \log |d|}(1-2 \epsilon)\right)+e^{-\frac{\epsilon}{4} V \log V}
$$

Proof. Set $x=|d|^{\frac{1}{\epsilon V}}$ and $z=x^{1 / \log \log |d|}$. Let

$$
P(\Lambda ; x)=P_{1}(\Lambda)+P_{2}(\Lambda)+P_{3}(\Lambda)
$$

where

$$
P_{1}(\Lambda)=\sum_{\substack{p \leq z \\ p \nmid d, p \nmid N}} \frac{a_{\pi}(p) a_{\mathcal{A I}(\Lambda)}(p)}{p^{\frac{1}{2}+\frac{1}{\log x}}} \frac{\log x / p}{\log x}, \quad P_{2}(\Lambda)=\sum_{\substack{z<p \leq x \\ p \nmid d, p \nmid N}} \frac{a_{\pi}(p) a_{\mathcal{A I}(\Lambda)}(p)}{p^{\frac{1}{2}+\frac{1}{\log x}}} \frac{\log x / p}{\log x},
$$

and $P_{3}(\Lambda)=P(\Lambda ; x)-P_{1}(\Lambda)-P_{2}(\Lambda)$. Let $V_{1}=(1-\epsilon) V, V_{2}=\frac{\epsilon}{2} V$. Clearly, if $P(\Lambda ; x)>V$, then (i) $P_{1}(\Lambda)>V_{1}$, (ii) $P_{2}(\Lambda)>V_{2}$, or (iii) $P_{3}(\Lambda)>V_{2}$. By Lemma 6.6 we get that the number of $\Lambda \in \widehat{\mathrm{Cl}_{K}}$ such that (i) holds is bounded by

$$
\frac{1}{V_{1}^{2 \ell}} \sum_{\Lambda \in \widehat{\mathrm{Cl}}_{K}} P_{1}(\Lambda)^{2 \ell} \ll\left|\mathrm{Cl}_{K}\right| \frac{(2 \ell) !}{\left(V_{1}^{2} 2\right)^{\ell} \ell !}\left(2 \sum_{\substack{p \leq d \\\left(\frac{d}{p}\right)=1, p \nmid N}} \frac{a_{\pi}(p)^{2}}{p}\right)^{\ell}
$$


provided that $\ell \leq \frac{1}{3} \epsilon V \log \log |d|$. After application of Lemma 6.4 and Stirling's formula, the right-hand side is $\ll_{\pi, \epsilon}\left|\mathrm{Cl}_{K}\right|\left(\frac{2 \ell \log \log |d|\left(1+\epsilon^{3}\right)}{V_{1}^{2} e}\right)^{\ell}$. For $V \leq \frac{1}{3} \epsilon(\log \log |d|)^{2}$, set $\ell=$ $\left\lfloor\frac{V_{1}^{2}}{2 \log \log |d|}\right\rfloor$, and for larger $V$, take $\ell=\lfloor\epsilon V / 3\rfloor$. Hence

$$
\frac{1}{\left|\mathrm{Cl}_{K}\right|}\left|\left\{\Lambda \in \widehat{\mathrm{Cl}_{K}}: P_{1}(\Lambda)>V_{1}\right\}\right| \ll_{\pi, \epsilon} \exp \left(\frac{-V^{2}(1-2 \epsilon)}{2 \log \log |d|}\right)+e^{-\frac{\epsilon}{4} V \log V}
$$

Arguing as before, taking $\ell=\lfloor\epsilon V / 3\rfloor$, and using the bound $\left|a_{\pi}(p)\right|^{2} \leq 16$, we have

$$
\begin{aligned}
\frac{1}{\left|\mathrm{Cl}_{K}\right|}\left|\left\{\Lambda \in \widehat{\mathrm{Cl}}_{K}: P_{2}(\Lambda)>V_{2}\right\}\right| & \ll \frac{(2 \ell) !}{\left(V_{2}^{2} 2\right)^{\ell} \ell !}\left(2 \sum_{\substack{z \leq p \leq x \\
p \nmid N}} \frac{a_{\pi}(p)^{2}}{p}\right)^{\ell} \\
& \ll\left(\frac{65 \ell \log \log \log |d|}{V_{2}^{2}}\right)^{\ell} \ll_{\epsilon} e^{-\frac{\epsilon}{4} V \log V .}
\end{aligned}
$$

Similarly,

$$
\frac{1}{\left|\mathrm{Cl}_{K}\right|}\left|\left\{\Lambda \in \widehat{\mathrm{Cl}_{K}}: P_{3}(\Lambda)>V_{2}\right\}\right| \ll \frac{(2 \ell) !}{\left(V_{2}^{2} 2\right)^{\ell} \ell !}\left(\sum_{p \mid d, p \nmid N} \frac{a_{\pi}(p)^{2}}{p}\right)^{\ell} \ll_{\epsilon} e^{-\frac{\epsilon}{4} V \log V} .
$$

Combining the bounds (64)-(66) completes the proof.

\subsection{Proof of Theorem 6.1}

Let $B_{K}(V)=\frac{1}{\left|\mathrm{Cl}_{K}\right|}\left|\left\{\Lambda \in \widehat{\mathrm{Cl}}_{K}: \log L\left(\frac{1}{2}, \pi \times \mathcal{A} \mathcal{I}(\Lambda)\right)>V\right\}\right|$. Observe that

$$
\begin{aligned}
\frac{1}{\left|\mathrm{Cl}_{K}\right|} \sum_{\Lambda \in \widehat{\mathrm{Cl}_{K}}} \sqrt{L\left(\frac{1}{2}, \pi \times \mathcal{A I}(\Lambda)\right)} & =\frac{1}{2} \int_{\mathbb{R}} e^{V / 2} B_{K}(V) d V \\
& =\frac{1}{2(\log |d|)^{1 / 4}} \int_{\mathbb{R}} e^{V / 2} B_{K}\left(V-\frac{1}{2} \log \log |d|\right) d V .
\end{aligned}
$$

It suffices to consider $2(\log \log |d|)^{3 / 4} \leq V \leq 2 C_{0} \frac{\log |d|}{\log \log |d|}$, as the contribution of $V \leq$ $2(\log \log |d|)^{3 / 4}$ is trivially $O_{\varepsilon}\left((\log |d|)^{-1 / 4+\varepsilon}\right)$, and by Remark 6.3 we have $B_{K}(V)=0$ for $V \geq 2 C_{0} \frac{\log |d|}{\log \log |d|}$.

By Lemmas 6.2 and 6.4 and the bound (59), we have for $x \leq|d|$ that

$$
\log L\left(\frac{1}{2}, \pi \times \mathcal{A} \mathcal{I}(\Lambda)\right) \leq P(\Lambda ; x)-\frac{1}{2} \log \log |d|+2 C_{0} \frac{\log |d|}{\log x}+o(\log \log |d|) .
$$

Taking $x=|d|^{\frac{1}{\epsilon V}}$, it follows from the bound (68) that $B_{K}\left(V-\frac{1}{2} \log \log |d|\right) \leq$ $A_{K}\left(V\left(1-3 C_{0} \epsilon\right) ;|d|^{\frac{1}{\epsilon V}}\right)$. Applying the foregoing estimate and using Lemma 6.7 along 
with the identity

$$
\int_{\mathbb{R}} e^{\frac{-t^{2}}{2 \log \log |d|}+t / 2} d t=\sqrt{2 \pi \log \log |d|}(\log |d|)^{\frac{1}{8}}
$$

we get that the right-hand side of equation (67) is

$$
\begin{aligned}
& \ll(\log |d|)^{-\frac{1}{4}} \int_{2(\log \log |d|)^{3 / 4}}^{2 C_{0} \frac{\log |d|}{\log \log |d|}}\left(e^{\frac{-V^{2}\left(1-7 C_{0} \varepsilon\right)}{2 \log \log |d|}}+e^{-\frac{\epsilon}{5} V \log V}\right) d V \\
& +(\log |d|)^{-\frac{1}{4}+\varepsilon} \ll_{\pi, \varepsilon} \frac{1}{(\log |d|)^{\frac{1}{8}-\varepsilon}},
\end{aligned}
$$

which completes the proof.

Acknowledgments. We thank Ralf Schmidt for helpful discussions concerning the material in §5.4. We thank Valentin Blomer and Farrell Brumley for forwarding us their preprint [5]. We thank the anonymous referee for useful comments and corrections which improved this paper. This work was supported by the Engineering and Physical Sciences Research Council (grant EP/T028343/1).

Competing Interest. The author(s) declare none.

\section{References}

[1] A. N. Andrianov, Euler products that correspond to Siegel's modular forms of genus 2, Uspekhi Mat. Nauk 29(3) (1974), 43-110.

[2] J. Arthur, The Endoscopic Classification of Representations, American Mathematical Society Colloquium Publications, 61 (American Mathematical Society, Providence, RI, 2013).

[3] A. O. L. Atkin And J. Lehner, Hecke operators on $\Gamma_{0}(m)$, Math. Ann. 185 (1970), $134-160$.

[4] T. Berger, L. Dembélé, A. Pacetti and M. H. Şengün, Theta lifts of Bianchi modular forms and applications to paramodularity, J. Lond. Math. Soc. (2) 92(2) (2015), 353-370.

[5] V. Blomer and F. Brumley, 'Simultaneous equidistribution of toric periods and fractional moments of $L$-functions', Preprint, 2020, URL: arXiv:2009.07093.

[6] S. Böcherer, Bemerkungen über die Dirichletreihen von Koecher und Maass, Mathematica Gottingensis 68 (Georg-August University, Göttingen 1986), 36 pp.

[7] S. Böcherer And S. DAS, On fundamental Fourier coefficients of Siegel modular forms, J. Inst. Math. Jussieu (2021), 1-41. doi.org/10.1017/S1474748021000086

[8] A. Borel, Admissible representations of a semi-simple group over a local field with vectors fixed under an Iwahori subgroup, Invent. Math. 35 (1976), 233-259.

[9] D. Bump And D. Ginzburg, Symmetric square $L$-functions on GL(r), Ann. of Math. (2) 136(1) (1992), 137-205.

[10] P.-S. Chan and W. T. Gan, The local Langlands conjecture for GSp(4) III: Stability and twisted endoscopy, J. Number Theory 146 (2015), 69-133.

[11] V. Chandee, Explicit upper bounds for $L$-functions on the critical line, Proc. Amer. Math. Soc. 137(12) (2009), 4049-4063. 
[12] Y. Choie, S. Gun And W. Kohnen, An explicit bound for the first sign change of the Fourier coefficients of a Siegel cusp form, Int. Math. Res. Not. IMRN 2015(12) (2015), 3782-3792.

[13] S. DAS, Omega results for Fourier coefficients of half-integral weight and Siegel modular forms, in International Conference on Number Theory, pp. 59-72 (Springer, Singapore, 2020).

[14] S. Das and W. Kohnen, On sign changes of eigenvalues of Siegel cusp forms of genus 2 in prime powers, Acta Arith. 183(2) (2018), 167-172.

[15] M. Dickson, A. Pitale, A. Saha and R. Schmidt, Explicit refinements of Böcherer's conjecture for Siegel modular forms of squarefree level, J. Math. Soc. Japan 72(1) (2020), 251-301.

[16] M. Eichler and D. Zagier, The Theory of Jacobi Forms, Progress in Mathematics, 55 (Birkhäuser Boston Inc., Boston, MA, 1985).

[17] M. Furusawa, On $L$-functions for $\mathrm{GSp}(4) \times \mathrm{GL}(2)$ and their special values, J. Reine Angew. Math. 438 (1993), 187-218.

[18] M. Furusawa and K. Morimoto, Refined global Gross-Prasad conjecture on special Bessel periods and Böcherer's conjecture, J. Eur. Math. Soc. (JEMS) 23(4) (2021), 12951331.

[19] W. T. Gan And S. Takeda, The local Langlands conjecture for GSp(4), Ann. of Math. (2) $\mathbf{1 7 3}(3)$ (2011), 1841-1882.

[20] V. GRItsenko, Analytic continuation of symmetric squares, Math. USSR Sb. 35(5) (1979), 593-614.

[21] S. Gun, W. Kohnen and K. Soundararajan, 'Large Fourier coefficients of half-integer weight modular forms', Preprint, 2020, arXiv:2004.14450.

[22] S. Gun And J. Sengupta, Sign changes of Fourier coefficients of Siegel cusp forms of degree two on Hecke congruence subgroups, Int. J. Number Theory 13(10) (2017), 25972625.

[23] G. Harcos, An additive problem in the Fourier coefficients of cusp forms, Math. Ann. 326(2) (2003), 347-365.

[24] X. He and L. ZhaO, On the first sign change of Fourier coefficients of cusp forms, $J$. Number Theory 190 (2018), 212-228.

[25] D. R. Heath-Brown, A mean value estimate for real character sums, Acta Arith. 72(3) (1995), 235-275.

[26] G. Henniart, Sur la fonctorialité, pour GL(4), donnée par le carré extérieur, Mosc. Math. J. 9(1) (2009), 33-45.

[27] B. Huang And S. Lester, 'Quantum variance for dihedral Maass forms', Preprint, 2020, arXiv:2007.02055.

[28] H. Iwaniec, Primes represented by quadratic polynomials in two variables, Acta Arith. $24(1973 / 74), 435-459$.

[29] J. Johnson-Leung And B. Roberts, Siegel modular forms of degree two attached to Hilbert modular forms, J. Number Theory 132(4) (2012), 543-564.

[30] M. Jutila, Tranformations of exponential sums, in Proceedings of the Amalfi Conference on Analytic Number Theory (Maiori, 1989), pp. 263-270 (University of Salerno, Salerno, 1992).

[31] J. Kaczorowski and A. Perelli, On the prime number theorem for the Selberg class, Arch. Math. (Basel) 80(3) (2003), 255-263.

[32] H. KIM, Functoriality for the exterior square of $\mathrm{GL}_{4}$ and the symmetric fourth of $\mathrm{GL}_{2}, J$. Amer. Math. Soc. 16(1) (2003), 139-183, with appendices by D. Ramakrishnan, H. Kim and P. Sarnak.

[33] W. Kohnen, Newforms of half-integral weight, J. Reine Angew. Math. 333 (1982), 32-72. 
[34] W. Kohnen, Fourier coefficients of modular forms of half-integral weight, Math. Ann. 271(2) (1985), 237-268.

[35] W. Kohnen, Estimates for Fourier coefficients of Siegel cusp forms of degree two, Compos. Math. 87(2) (1993), 231-240.

[36] W. Kohnen, Sign changes of Fourier coefficients and eigenvalues of cusp forms, in Number Theory, Ser. Number Theory Appl., vol. 2, pp. 97-107 (World Scientifice Publishing, Hackensack, NJ, 2007).

[37] E. M. LAPID, On the nonnegativity of Rankin-Selberg $L$-functions at the center of symmetry, Int. Math. Res. Not. IMRN 2003(2) (2003), 65-75.

[38] S. Lester AND M. RADZIWiet, Quantum unique ergodicity for half-integral weight automorphic forms, Duke Math. J. 169(2) (2020), 279-351.

[39] S. Lester and M. RadziwiŁe, Signs of Fourier coefficients of half-integral weight modular forms, Math. Ann. 379(3-4) (2021), 1553-1604.

[40] Y. Liu, Refined global Gan-Gross-Prasad conjecture for Bessel periods, J. Reine Angew. Math. 717 (2016), 133-194.

[41] M. Manickam and B. Ramakrishnan, On Shimura, Shintani and Eichler-Zagier correspondences, Trans. Amer. Math. Soc. 352(6) (2000), 2601-2617.

[42] K. MatomäKi And M. RadziwiŁe, Sign changes of Hecke eigenvalues, Geom. Funct. Anal. 25(6) (2015), 1937-1955.

[43] M. B. Milinovich and C. L. Turnage-Butterbaugh, Moments of products of automorphic L-functions, J. Number Theory 139 (2014), 175-204.

[44] A. Pitale, A. Saha and R. Schmidt, Transfer of Siegel cusp forms of degree 2, Mem. Amer. Math. Soc. 232(1090) (2014).

[45] A. Pitale and R. Schmidt, Sign changes of Hecke eigenvalues of Siegel cusp forms of degree 2, Proc. Amer. Math. Soc. 136(11) (2008), 3831-3838.

[46] A. Pitale and R. Schmidt, Integral representation for $L$-functions for GSp4 $\times$ GL2, $J$. Number Theory 129(10) (2009), 1272 - 1324.

[47] A. Pitale and R. Schmidt, Ramanujan-type results for Siegel cusp forms of degree 2, J. Ramanujan Math. Soc. 24(1) (2009), 87-111.

[48] D. Prasad and D. Ramakrishnan, On the global root numbers of $\mathrm{GL}(n) \times \mathrm{GL}(m)$, in Automorphic Forms, Automorphic Representations, and Arithmetic (Fort Worth, TX, 1996), Proceedings of Symposia in Pure Mathematics, 66, pp. 311-330 (American Mathematical Societ, Providence, RI, 1999).

[49] Y. QIU, 'The Bessel period functional on SO(5): The nontempered case, Preprint, 2013, URL: arXiv:1312.5793.

[50] M. Radziwile and K. Soundararajan, Moments and distribution of central $L$-values of quadratic twists of elliptic curves, Invent. Math. 202(3) (2015), 1029-1068.

[51] H. Resnikoff and R. L. Saldana, Some properties of Fourier coefficients of Eisenstein series of degree two, J. Reine Angew. Math. 265 (1974), 90-109.

[52] B. Roberts And R. Schmidt, Local Newforms for GSp(4), Lecture Notes in Mathematics, 1918 (Springer, Berlin, 2007).

[53] E. Royer, J. Sengupta And J. Wu, Non-vanishing and sign changes of Hecke eigenvalues for Siegel cusp forms of genus two, Ramanujan J. 39(1) (2016), 179-199, with an appendix by E. Kowalski and A. Saha.

[54] A. SAHA, $L$-functions for holomorphic forms on $\mathrm{GSp}(4) \times \mathrm{GL}(2)$ and their special values, Int. Math. Res. Not. IMRN 2009(10) (2009), 1773-1837.

[55] A. SaHA, Pullbacks of Eisenstein series from GU $(3,3)$ and critical $L$-values for GSp4 $\times$ GL2, Pacific J. Math. 246(2) (2010), 435-486.

[56] A. SAHA, Siegel cusp forms of degree 2 are determined by their fundamental Fourier coefficients, Math. Ann. 355(1) (2013), 363-380. 
[57] A. SAhA, On ratios of Petersson norms for Yoshida lifts, Forum Math. 27(4) (2015), 2361-2412.

[58] A. Saha And R. Schmidt, Yoshida lifts and simultaneous non-vanishing of dihedral twists of modular L-functions, J. Lond. Math. Soc. (2) 88 (2013), 251-270.

[59] R. Schмidt, Packet structure and paramodular forms, Trans. Amer. Math. Soc. 370(5) (2018), 3085-3112.

[60] F. Shahidi, On certain L-functions, Amer. J. Math. 103(2) (1981), 297-355.

[61] F. Shahidi, On non-vanishing of twisted symmetric and exterior square $L$-functions for GL(n), Pacific J. Math., Special Issue, 1997, Olga Taussky-Todd: in memoriam, pp. 311-322.

[62] G. Shimura, On modular forms of half integral weight, Ann. of Math. (2) 97 (1973), 440-481.

[63] K. Soundararajan, Extreme values of zeta and L-functions, Math. Ann. 342(2) (2008), 467-486.

[64] K. Soundararajan, Moments of the Riemann zeta function, Ann. of Math. (2) 170(2) (2009), 981-993.

[65] K. Soundararajan And M. P. Young, The second moment of quadratic twists of modular L-functions, J. Eur. Math. Soc. (JEMS) 12(5) (2010), 1097-1116.

[66] S. TAKEDA, On a certain metaplectic Eisenstein series and the twisted symmetric square $L$-function, Math. Z. 281(1-2) (2015), 103-157.

[67] J.-L. WALDSpurger, Sur les valeurs de certaines fonctions $L$ automorphes en leur centre de symétrie, Compos. Math. 54(2) (1985), 173-242.

[68] R. Weissauer, Endoscopy for GSp(4) and the Cohomology of Siegel Modular Threefolds, Lecture Notes in Mathematics, 1968 (Springer-Verlag, Berlin, 2009).

[69] J. Wu AND Y. YE, Hypothesis $\mathrm{H}$ and the prime number theorem for automorphic representations, Funct. Approx. Comment. Math. 37(2) (2007), 461-471. 